\title{
ANALYSIS OF THE VELOCITY TRACKING CONTROL PROBLEM FOR THE 3D EVOLUTIONARY NAVIER-STOKES EQUATIONS*
}

\author{
EDUARDO CASAS $^{\dagger}$ AND KONSTANTINOS CHRYSAFINOS ${ }^{\ddagger}$
}

\begin{abstract}
The velocity tracking problem for the evolutionary Navier-Stokes equations in three dimensions is studied. The controls are of distributed type and are submitted to bound constraints. The classical cost functional is modified so that a full analysis of the control problem is possible. First and second order necessary and sufficient optimality conditions are proved. A fully discrete scheme based on a discontinuous (in time) Galerkin approach, combined with conforming finite element subspaces in space, is proposed and analyzed. Provided that the time and space discretization parameters, $\tau$ and $h$, respectively, satisfy $\tau \leq C h^{2}$, the $L^{2}\left(\Omega_{T}\right)$ error estimates of order $O(h)$ are proved for the difference between the locally optimal controls and their discrete approximations. Finally, combining these techniques and the approach of Casas, Herzog, and Wachsmuth [SIAM J. Optim., 22 (2012), pp. 795-820], we extend our results to the case of $L^{1}\left(\Omega_{T}\right)$ type functionals that allow sparse controls.
\end{abstract}

Key words. evolution Navier-Stokes equations, optimal control, sparse controls, a priori error estimates, discontinuous Galerkin methods

AMS subject classifications. 49J20, 49K20, 35K55, 65N30

DOI. $10.1137 / 140978107$

1. Introduction. In this paper, we study the following velocity tracking control problem associated to the evolutionary Navier-Stokes equations for three-dimensional (3D) flows:

$$
\left\{\begin{array}{l}
\mathbf{y}_{t}-\nu \Delta \mathbf{y}+(\mathbf{y} \cdot \nabla) \mathbf{y}+\nabla p=\mathbf{f}+\mathbf{u} \text { in } \Omega_{T}=(0, T) \times \Omega, \\
\operatorname{div} \mathbf{y}=0 \text { in } \Omega_{T}, \quad \mathbf{y}(0)=\mathbf{y}_{0} \text { in } \Omega, \quad \mathbf{y}=0 \text { on } \Sigma_{T}=(0, T) \times \Gamma .
\end{array}\right.
$$

In these equations, $\mathbf{y}=\left(y_{1}, y_{2}, y_{3}\right)$ is the velocity field of the fluid, $p$ is the pressure, $\nu>0$ is the viscosity, $\mathbf{f}$ and $\mathbf{u}$ represent the body forces, and $\mathbf{y}_{0}$ denotes the initial velocity. We can control the system through the forces $\mathbf{u}$.

For two-dimensional (2D) flows, $\Omega \subset \mathbb{R}^{2}$, an existence and uniqueness theorem for a solution of (1.1) has been known for a long time. The study is more complicated for the $3 \mathrm{D}$ flows, $\Omega \subset \mathbb{R}^{3}$. In this case, two different types of solutions are distinguished: weak and strong. Under minimal assumptions, the existence of weak solutions $\mathbf{y} \in$ $L^{2}\left(0, T ; \mathbf{H}_{0}^{1}(\Omega)\right) \cap C_{w}\left([0, T], \mathbf{L}^{2}(\Omega)\right)$ can be proved. However, the uniqueness is still an open problem, unless the data $\left(\mathbf{f}+\mathbf{u}, \mathbf{y}_{0}\right)$ are small enough or final time $T$ is sufficiently small; see, for instance, Temam [21].

A strong solution $\mathbf{y}$ is a weak solution that additionally belongs to $L^{8}\left(0, T ; \mathbf{L}^{4}(\Omega)\right)$. In the 3D case, there exists at most one strong solution of (1.1), but its existence has not been proved until now. In the 2D case, weak and strong solutions coincide, and hence we have existence and uniqueness of a solution.

\footnotetext{
* Received by the editors July 18, 2014; accepted for publication (in revised form) October 28, 2015; published electronically January 14, 2016.

http://www.siam.org/journals/sicon/54-1/97810.html

†Departamento de Matemática Aplicada y Ciencias de la Computación, E.T.S.I. Industriales y de Telecomunicación, Universidad de Cantabria, Av. Los Castros s/n, 39005 Santander, Spain (eduardo. casas@unican.es). This author was partially supported by the Spanish Ministerio de Economía y Competitividad under projects MTM2011-22711 and MTM2014-57531-P.

$\ddagger$ Department of Mathematics, School of Applied Mathematics and Physical Sciences, National Technical University of Athens, Zografou Campus, Athens 15780, Greece (chrysafinos@math.ntua. gr).
} 
In the classical tracking control problem, the cost functional involves the $L^{2}$ norm of $\mathbf{y}-\mathbf{y}_{d}$, where $\mathbf{y}_{d}$ is the given target field. In the case of $3 \mathrm{D}$ flows, due to the lack of uniqueness of weak solutions or of the existence of strong solutions, the analysis is very complicated. Actually, we cannot prove first and second order optimality conditions, and error estimates for the discretization of the control problem is an open issue. As a consequence, most of the studies devoted to the control problems associated to the equations (1.1) assume that $\Omega \subset \mathbb{R}^{2}[1,5,9,11,12,14,22]$.

Hereafter, we assume $\Omega \subset \mathbb{R}^{3}$, but we do not require the data to be small because this is not a realistic assumption. In this paper, we deal with strong solutions, which allows us to carry out a complete analysis of the control problem. However, to prove the existence of an optimal control with an associated strong solution, we have to consider a convenient cost functional. Instead of setting the $L^{2}$ norm of $\mathbf{y}-\mathbf{y}_{d}$ in the cost functional as usual, we consider the functional

$$
\begin{aligned}
\mathcal{J}(\mathbf{u}, \mathbf{y})= & \frac{1}{8} \int_{0}^{T}\left\|\mathbf{y}(t)-\mathbf{y}_{d}(t)\right\|_{\mathbf{L}^{4}(\Omega)}^{8} d t \\
& +\frac{\gamma}{2} \int_{\Omega}\left|\mathbf{y}(T, x)-\mathbf{y}_{\Omega}(x)\right|^{2} d x+\frac{\lambda}{2} \int_{0}^{T} \int_{\Omega}|\mathbf{u}(t, x)|^{2} d x \mathrm{~d} t,
\end{aligned}
$$

where $\lambda>0, \gamma \geq 0$, and $\mathbf{y}_{\Omega} \in \mathbf{L}^{2}(\Omega)$ to be fixed more precisely later.

The goal is to minimize the $\mathcal{J}(\mathbf{u}, \mathbf{y})$ in a certain class of functions, where $(\mathbf{u}, \mathbf{y})$ satisfies (1.1). If $\mathbf{y}$ is a weak solution of (1.1) such that $\mathcal{J}(\mathbf{u}, \mathbf{y})<+\infty$, then $\mathbf{y}$ is a strong solution. With this formulation we can prove the existence of an optimal control and get the first and second order optimality conditions. Moreover, following the approach of [5], we obtain the same error estimates proved there for the numerical discretization of the control problem in three dimensions. In particular, we prove estimates of order $\mathcal{O}(h)$ for the difference between locally optimal controls and their discrete approximations, for $\tau \leq C h^{2}$, when $\tau, h$ denote the time and space discretization parameters, respectively. In addition, we also show that any strict local minimum can be approximated by a sequence of local minima of the discrete optimal control problems. Estimates of order $\mathcal{O}(h)$ are also obtained for the state and adjoint variables, and they are optimal in terms of the regularity on the given data. The cost functional (1.2) was introduced in [4, p. 95], where existence of optimal controls and first order optimality conditions were studied for the continuous problem. It is worth noting that it plays a crucial role also in the development of error estimates when combined with the discontinuous (in time) Galerkin framework. One of the main features of discontinuous (in time) Galerkin machinery is that the discrete scheme inherits regularity properties of the corresponding continuous problem due to its heavily implicit nature. In particular, the fact that the cost functional (1.2) yields strong solutions is an important asset at the fully discrete level, since the enhanced regularity is also inherited by the discrete state and adjoint variables. As a consequence, it allows the numerical analysis of the control to state and adjoint mappings similarly to the 2D case and as in [5].

Furthermore, we also discuss the case of sparse controls. To enforce sparsity of the controls, i.e., the localization of the controls in a small region of the domain, we modify our functional in a way to include the $L^{1}\left(\Omega_{T}\right)$ norm. It is well understood that the inclusion of the $L^{1}$ norm in the cost functional yields sparse controls (see, for instance, $[6,7,13,20,23])$. In [6] necessary and sufficient second order optimality conditions are derived for a semilinear elliptic control problem. Adopting the techniques of [6] in our optimal control setting for the 3D evolutionary Navier-Stokes case, we also 
prove error estimates for the difference between the locally optimal controls and their discrete approximations based on the discontinuous (in time) Galerkin framework.

2. The state equation. Assumptions and preliminary results. Hereafter $\Omega$ denotes a bounded open subset in $\mathbb{R}^{3}$ with a Lipschitz boundary $\Gamma$. We assume that either $\Omega$ is convex or $\Gamma$ is of class $C^{1,1}$. The outward unit normal vector to $\Gamma$ at a point $x \in \Gamma$ is denoted by $\mathbf{n}(x)$. Given $0<T<+\infty$, we set $\Omega_{T}=(0, T) \times \Omega$ and $\Sigma_{T}=(0, T) \times \Gamma$. As in [5], we denote the Sobolev spaces $\mathbf{H}^{1}(\Omega)=H^{1}\left(\Omega ; \mathbb{R}^{3}\right)$, $\mathbf{H}_{0}^{1}(\Omega)=H_{0}^{1}\left(\Omega ; \mathbb{R}^{3}\right), \mathbf{H}^{-1}(\Omega)=\left(\mathbf{H}_{0}^{1}(\Omega)\right)^{\prime}$, and $\mathbf{W}^{s, p}(\Omega)=W^{s, p}\left(\Omega ; \mathbb{R}^{3}\right)$ for $1 \leq p \leq \infty$ and $s>0$. We also consider the spaces of integrable functions

$$
L_{0}^{2}(\Omega)=\left\{w \in L^{2}(\Omega): \int_{\Omega} w(x) \mathrm{d} x=0\right\} ;
$$

$\mathbf{L}^{p}(\Omega)=L^{p}\left(\Omega ; \mathbb{R}^{3}\right)$, and, for a given Banach space $X, L^{p}(0, T ; X)$ will denote the integrable functions defined in $(0, T)$ and taking values in $X$ endowed with the usual norm. Following Lions and Magenes [17, Vol. 1] we put

$$
H^{2,1}\left(\Omega_{T}\right)=\left\{y \in L^{2}\left(\Omega_{T}\right): \frac{\partial y}{\partial x_{i}}, \frac{\partial^{2} y}{\partial x_{i} x_{j}}, \frac{\partial y}{\partial t} \in L^{2}\left(\Omega_{T}\right), 1 \leq i, j \leq 2\right\} .
$$

Endowed with the standard norm, the space $H^{2,1}\left(\Omega_{T}\right)$ is continuously embedded in $C\left([0, T], H^{1}(\Omega)\right)$. We also set $\mathbf{H}^{2,1}\left(\Omega_{T}\right)=\left[H^{2,1}\left(\Omega_{T}\right)\right]^{3}$. We introduce the usual spaces of divergence-free vector fields,

$$
\begin{aligned}
& \mathbf{Y}=\left\{\mathbf{y} \in \mathbf{H}_{0}^{1}(\Omega): \operatorname{div} \mathbf{y}=0 \text { in } \Omega\right\}, \\
& \mathbf{H}=\left\{\mathbf{y} \in \mathbf{L}^{2}(\Omega): \operatorname{div} \mathbf{y}=0 \text { in } \Omega \text { and } \mathbf{y} \cdot \mathbf{n}=0 \text { on } \Gamma\right\} .
\end{aligned}
$$

Finally, let us consider the space $\mathbf{W}(0, T)=\left\{\mathbf{y} \in L^{2}(0, T ; \mathbf{Y}): \mathbf{y}_{t} \in L^{2}\left(0, T ; \mathbf{Y}^{*}\right)\right\}$. It is well known that $\mathbf{W}(0, T) \subset C_{w}([0, T], \mathbf{H})$, where $C_{w}([0, T], \mathbf{H})$ is the space of weakly continuous functions $\mathbf{y}:[0, T] \longrightarrow \mathbf{H}$.

Throughout this paper, we will assume that $\mathbf{f}, \mathbf{u} \in L^{2}\left(0, T ; \mathbf{L}^{2}(\Omega)\right)$ and $\mathbf{y}_{0}, \mathbf{y}_{\Omega} \in$ $\mathbf{Y}$. An element $\mathbf{y} \in \mathbf{W}(0, T)$ is said to be a weak solution of (1.1) if

$$
\left\{\begin{array}{l}
\left(\mathbf{y}_{t}, \boldsymbol{\psi}\right)+a(\mathbf{y}, \boldsymbol{\psi})+c(\mathbf{y}, \mathbf{y}, \boldsymbol{\psi})=(\mathbf{f}+\mathbf{u}, \boldsymbol{\psi}) \forall \boldsymbol{\psi} \in \mathbf{Y} \text { for a.a. } t \in[0, T] \\
\mathbf{y}(0)=\mathbf{y}_{0}
\end{array}\right.
$$

and the following energy inequality holds:

$$
\|\mathbf{y}(t)\|_{\mathbf{L}^{2}(\Omega)}^{2}+2 \nu \int_{0}^{t}\|\mathbf{y}(s)\|_{\mathbf{H}_{0}^{1}(\Omega)}^{2} d s \leq\left\|\mathbf{y}_{0}\right\|^{2}+2 \int_{0}^{t}(\mathbf{f}(s)+\mathbf{u}(s), \mathbf{y}(s)) d s \quad \forall t \in[0, T]
$$

where $\|\cdot\|$ and $(\cdot, \cdot)$ denote the norm and the inner product, respectively, in $\mathbf{L}^{2}(\Omega)$, and $a: \mathbf{H}^{1}(\Omega) \times \mathbf{H}^{1}(\Omega) \longrightarrow \mathbb{R}$ and $c: \mathbf{L}^{4}(\Omega) \times \mathbf{H}^{1}(\Omega) \times \mathbf{H}^{1}(\Omega) \longrightarrow \mathbb{R}$ are defined by

$$
\begin{aligned}
& a(\mathbf{y}, \mathbf{z})=\nu \int_{\Omega}(\nabla \mathbf{y}: \nabla \mathbf{z}) \mathrm{d} x=\nu \sum_{i, j=1}^{3} \int_{\Omega} \partial_{x_{i}} y_{j} \partial_{x_{i}} z_{j} \mathrm{~d} x, \\
& c(\mathbf{y}, \mathbf{z}, \mathbf{w})=\frac{1}{2}[\hat{c}(\mathbf{y}, \mathbf{z}, \mathbf{w})-\hat{c}(\mathbf{y}, \mathbf{w}, \mathbf{z})] \text { with } \hat{c}(\mathbf{y}, \mathbf{z}, \mathbf{w})=\sum_{i, j=1}^{3} \int_{\Omega} \mathbf{y}_{j}\left(\frac{\partial \mathbf{z}_{i}}{\partial x_{j}}\right) \mathbf{w}_{i} \mathrm{~d} x .
\end{aligned}
$$

The existence of a weak solution is well known; see, for instance, Ladyzhenskaya [15], Lions [16], Temam [21], etc. However, the uniqueness of a weak solution is an 
open question so far. We say that $\mathbf{y}$ is a strong solution of (1.1) if it is a weak solution and $\mathbf{y} \in L^{8}\left(0, T ; \mathbf{L}^{4}(\Omega)\right)$. It is well known that (1.1) does not have more than one strong solution. Strong solutions satisfy the energy equality instead of the energy inequality (2.2). Hence, they seem to be physically more significant than weak solutions. Unfortunately there is no existence result for strong solutions.

Once $\mathbf{y}$ is found from (2.1), the existence of the pressure $p \in \mathcal{D}^{\prime}\left(\Omega_{T}\right)$ can be proved in such a way that $(\mathbf{y}, p)$ is a solution of (1.1).

We finish this section by collecting some results, whose proofs can be found in [4].

Theorem 2.1. Let $\left(\mathbf{f}, \mathbf{y}_{0}\right) \in L^{2}\left(0, T ; \mathbf{L}^{2}(\Omega)\right) \times \mathbf{Y}, \mathbf{g} \in L^{8}\left(0, T ; \mathbf{L}^{4}(\Omega)\right)$ with $\operatorname{div} \mathbf{g}=0$ in $\Omega_{T}$, and $\mathbf{e} \in L^{\infty}(0, T ; \mathbf{Y}) \cap L^{3 / 2}\left(0, T ; \mathbf{H}^{2}(\Omega)\right)$. Then, there exist a unique element $\mathbf{y} \in \mathbf{H}^{2,1}\left(\Omega_{T}\right) \cap C([0, T], \mathbf{Y})$ and some $p \in L^{2}\left(0, T ; H^{1}(\Omega)\right)$ solution of the following problem:

$$
\left\{\begin{array}{l}
\frac{\partial \mathbf{y}}{\partial t}-\nu \Delta \mathbf{y}+(\mathbf{g} \cdot \nabla) \mathbf{y}+(\mathbf{y} \cdot \nabla) \mathbf{e}+\nabla p=\mathbf{f} \quad \text { in } \Omega_{T}, \\
\operatorname{div} \mathbf{y}=0 \quad \text { in } \Omega_{T}, \quad \mathbf{y}(0)=\mathbf{y}_{0} \quad \text { in } \Omega, \quad \mathbf{y}=0 \text { on } \Sigma_{T} .
\end{array}\right.
$$

Moreover, $p$ is unique up to the addition of a function of $L^{2}(0, T)$. Finally, there exists an increasing function $\eta:[0,+\infty) \longrightarrow[0,+\infty)$ depending only on $\Omega$ and $\nu$ such that

$$
\begin{gathered}
\|\mathbf{y}\|_{\mathbf{H}^{2,1}\left(\Omega_{T}\right)} \leq \eta\left(\left\|\mathbf{y}_{0}\right\|_{\mathbf{Y}}+\|\mathbf{f}\|_{L^{2}\left(0, T ; \mathbf{L}^{2}(\Omega)\right)}+\|\mathbf{g}\|_{L^{8}\left(0, T ; \mathbf{L}^{4}(\Omega)\right)}\right. \\
\left.+\|\mathbf{e}\|_{L^{\infty}(0, T ; \mathbf{Y})}+\|\mathbf{e}\|_{L^{3 / 2}\left(0, T ; \mathbf{H}^{2}(\Omega)\right)}\right) .
\end{gathered}
$$

Corollary 2.2. Let us assume that $(\mathbf{y}, p)$ is a strong solution of problem (1.1); then $\mathbf{y} \in \mathbf{H}^{2,1}\left(\Omega_{T}\right) \cap C([0, T], \mathbf{Y})$ and $p \in L^{2}\left(0, T ; H^{1}(\Omega)\right)$. Moreover,

$$
\|\mathbf{y}\|_{\mathbf{H}^{2,1}\left(\Omega_{T}\right)} \leq \eta\left(\left\|\mathbf{y}_{0}\right\|_{\mathbf{Y}}+\|\mathbf{f}+\mathbf{u}\|_{L^{2}\left(0, T ; \mathbf{L}^{2}(\Omega)\right)}+\|\mathbf{y}\|_{L^{8}\left(0, T ; \mathbf{L}^{4}(\Omega)\right)}\right),
$$

where $\eta$ is as in Theorem 2.1.

The proof of this corollary follows from Theorem 2.1 taking $\mathbf{g}=\mathbf{y}$ and $\mathbf{e}=0$.

COROLlaRY 2.3. If problem (1.1) has a strong solution for some element $\overline{\mathbf{u}} \in$ $L^{2}\left(0, T ; \mathbf{L}^{2}(\Omega)\right)$, then there exists an open neighborhood $\mathcal{A}_{0}$ of $\overline{\mathbf{u}}$ in $L^{2}\left(0, T ; \mathbf{L}^{2}(\Omega)\right)$ such that (1.1) has a strong solution for every $\mathbf{u} \in \mathcal{A}_{0}$. Moreover, the mapping $G: \mathcal{A}_{0} \longrightarrow \mathbf{H}^{2,1}\left(\Omega_{T}\right) \cap C([0, T], \mathbf{Y})$, defined by $G(\mathbf{u})=\mathbf{y}_{\mathbf{u}}$, is of class $C^{\infty}$. Finally, if $\mathbf{z}_{\mathbf{v}}=G^{\prime}(\mathbf{u}) \mathbf{v}$ and $\mathbf{v}_{\mathbf{v}_{1} \mathbf{v}_{2}}=G^{\prime \prime}(\mathbf{u})\left(\mathbf{v}_{1}, \mathbf{v}_{2}\right)$, for some $\mathbf{u} \in \mathcal{A}_{0}$ and some $\mathbf{v}, \mathbf{v}_{1}, \mathbf{v}_{2} \in$ $L^{2}\left(0, T ; \mathbf{L}^{2}(\Omega)\right)$, then $\mathbf{z}_{\mathbf{v}}$ and $\mathbf{z}_{\mathbf{v}_{1} \mathbf{v}_{2}}$ are the unique strong solutions of the following problems:

$$
\left\{\begin{array}{l}
\frac{\partial \mathbf{z}_{\mathbf{v}}}{\partial t}-\nu \Delta \mathbf{z}_{\mathbf{v}}+\left(\mathbf{y}_{\mathbf{u}} \cdot \nabla\right) \mathbf{z}_{\mathbf{v}}+\left(\mathbf{z}_{\mathbf{v}} \cdot \nabla\right) \mathbf{y}_{\mathbf{u}}+\nabla p_{1}=\mathbf{v} \quad \text { in } \Omega_{T}, \\
\operatorname{div} \mathbf{z}_{\mathbf{v}}=0 \quad \text { in } \Omega_{T}, \quad \mathbf{z}_{\mathbf{v}}(0)=0 \quad \text { in } \Omega, \quad \mathbf{z}_{\mathbf{v}}=0 \quad \text { on } \Sigma_{T},
\end{array}\right.
$$

$$
\left\{\begin{array}{l}
\frac{\partial \mathbf{z}_{\mathbf{v}_{1} \mathbf{v}_{2}}}{\partial t}-\nu \Delta \mathbf{z}_{\mathbf{v}_{1} \mathbf{v}_{2}}+\left(\mathbf{y}_{\mathbf{u}} \cdot \nabla\right) \mathbf{z}_{\mathbf{v}_{1} \mathbf{v}_{2}}+\left(\mathbf{z}_{\mathbf{v}_{1} \mathbf{v}_{2}} \cdot \nabla\right) \mathbf{y}_{\mathbf{u}} \\
\quad+\left(\mathbf{z}_{\mathbf{v}_{2}} \cdot \nabla\right) \mathbf{z}_{\mathbf{v}_{1}}+\left(\mathbf{z}_{\mathbf{v}_{1}} \cdot \nabla\right) \mathbf{z}_{\mathbf{v}_{2}}+\nabla p_{2}=0 \quad \text { in } \Omega_{T}, \\
\operatorname{div} \mathbf{z}_{\mathbf{v}_{1} \mathbf{v}_{2}}=0 \text { in } \Omega_{T}, \quad \mathbf{z}_{\mathbf{v}_{1} \mathbf{v}_{2}}(0)=0 \quad \text { in } \Omega, \quad \mathbf{z}_{\mathbf{v}_{1} \mathbf{v}_{2}}=0 \quad \text { on } \Sigma_{T}
\end{array}\right.
$$

for some $p_{1}, p_{2} \in L^{2}\left(0, T ; H^{1}(\Omega)\right)$, which are unique up to the addition of a function of $L^{2}(0, T)$. 
Proof. Here we modify the proof of [4, Cor. 4.2.2] to correct a small mistake. First, we observe that the solution $\mathbf{y} \in \mathbf{H}^{2,1}\left(\Omega_{T}\right) \cap C([0, T], \mathbf{Y})$ of $(2.3)$ satisfies that $\mathbf{y}^{\prime} \in L^{2}(0, T ; \mathbf{H})$. This is an immediate consequence of the proof of [4, Thm. 4.2.1]. Indeed, a Galerkin approach is followed there to approximate $\mathbf{y}$ by using a special basis $\left\{\boldsymbol{\psi}_{j}\right\}_{j=1}^{\infty}$ of $\mathbf{H}^{2}(\Omega) \cap \mathbf{Y}$. The approximations take the form $\mathbf{y}_{m}=\sum_{j=1}^{m} g_{j}(t) \boldsymbol{\psi}_{j}$, and $\mathbf{y}_{m}^{\prime}=\sum_{j=1}^{m} g_{j}^{\prime}(t) \boldsymbol{\psi}_{j}$ belonging to $L^{2}(0, T ; \mathbf{H})$ converges weakly to $\mathbf{y}^{\prime}$ in $L^{2}(0, T ; \mathbf{H})$.

Let us consider the space

$$
\mathcal{H}=\left\{\mathbf{y} \in \mathbf{H}^{2,1}\left(\Omega_{T}\right) \cap C([0, T], \mathbf{Y}): \mathbf{y}^{\prime} \in L^{2}(0, T ; \mathbf{H})\right\} .
$$

Endowed with the norm of $\mathbf{H}^{2,1}\left(\Omega_{T}\right)$, this is a Hilbert space. Now, we define the mapping

$$
\begin{aligned}
& F: \mathcal{H} \times L^{2}\left(0, T ; \mathbf{L}^{2}(\Omega)\right) \longrightarrow L^{2}(0, T ; \mathbf{H}) \times \mathbf{Y}, \\
& F(\mathbf{y}, \mathbf{u})=\left(\frac{\partial \mathbf{y}}{\partial t}+\mathbf{P}_{\mathbf{H}}[-\nu \Delta \mathbf{y}+(\mathbf{y} \cdot \nabla) \mathbf{y}-(\mathbf{f}+\mathbf{u})], \mathbf{y}(0)-\mathbf{y}_{0}\right),
\end{aligned}
$$

where $\mathbf{P}_{\mathbf{H}}: \mathbf{L}^{2}(\Omega) \longrightarrow \mathbf{H}$ denotes the projection operator. It is easy to check that $F$ is of class $C^{\infty}$ and

$$
\frac{\partial F}{\partial \mathbf{y}}(\mathbf{y}, \mathbf{u}) \mathbf{z}=\left(\frac{\partial \mathbf{z}}{\partial t}+\mathbf{P}_{\mathbf{H}}[-\nu \Delta \mathbf{z}+(\mathbf{y} \cdot \nabla) \mathbf{z}+(\mathbf{z} \cdot \nabla) \mathbf{y}], \mathbf{z}(0)\right) .
$$

Now, we observe that

$$
\begin{aligned}
& \left(\mathbf{P}_{\mathbf{H}}[-\nu \Delta \mathbf{z}+(\mathbf{y} \cdot \nabla) \mathbf{z}+(\mathbf{z} \cdot \nabla) \mathbf{y}], \boldsymbol{\psi}\right) \\
& =(-\nu \Delta \mathbf{z}+(\mathbf{y} \cdot \nabla) \mathbf{z}+(\mathbf{z} \cdot \nabla) \mathbf{y}, \boldsymbol{\psi})=a(\mathbf{y}, \boldsymbol{\psi})+c(\mathbf{y}, \mathbf{z}, \boldsymbol{\psi})+c(\mathbf{z}, \mathbf{y}, \boldsymbol{\psi}) \quad \forall \boldsymbol{\psi} \in \mathbf{Y} .
\end{aligned}
$$

Therefore, $\frac{\partial F}{\partial \mathbf{y}}(\mathbf{y}, \mathbf{u}) \mathbf{z}=\left(\mathbf{v}, \mathbf{z}_{0}\right)$, with $\left(\mathbf{v}, \mathbf{z}_{0}\right) \in L^{2}(0, T ; \mathbf{H}) \times \mathbf{Y}$, if and only if

$$
\left\{\begin{array}{l}
\left(\mathbf{z}_{t}, \boldsymbol{\psi}\right)+a(\mathbf{z}, \boldsymbol{\psi})+c(\mathbf{z}, \mathbf{y}, \boldsymbol{\psi})+c(\mathbf{y}, \mathbf{z}, \boldsymbol{\psi})=(\mathbf{v}, \boldsymbol{\psi}) \forall \boldsymbol{\psi} \in \mathbf{Y} \\
\mathbf{z}(0)=\mathbf{z}_{0}
\end{array}\right.
$$

or, equivalently,

$$
\left\{\begin{array}{l}
\frac{\partial \mathbf{z}}{\partial t}-\nu \Delta \mathbf{z}+(\mathbf{y} \cdot \nabla) \mathbf{z}+(\mathbf{z} \cdot \nabla) \mathbf{y}+\nabla p=\mathbf{v} \quad \text { in } \Omega_{T} \\
\operatorname{div} \mathbf{z}=0 \text { in } \Omega_{T}, \quad \mathbf{z}(0)=\mathbf{z}_{0} \quad \text { in } \Omega, \quad \mathbf{z}=0 \quad \text { on } \Sigma_{T}
\end{array}\right.
$$

for some $p \in L^{2}\left(0, T ; \mathbf{L}^{2}(\Omega)\right)$. Indeed, it is enough to recall that if $\mathbf{u}, \mathbf{v} \in L^{2}(0, T ; \mathbf{H})$ satisfy that $(\mathbf{v}, \boldsymbol{\psi})=(\mathbf{u}, \boldsymbol{\psi}) \forall \boldsymbol{\psi} \in \mathbf{Y}$, then $\mathbf{u}=\mathbf{v}$; see [10, Thm. 2.8, p. 30].

Now, with the help of Theorem 2.1 we infer that $\frac{\partial F}{\partial \mathbf{y}}(\mathbf{y}, \mathbf{u}): \mathcal{H} \longrightarrow L^{2}(0, T ; \mathbf{H}) \times \mathbf{Y}$ is an isomorphism for every $(\mathbf{y}, \mathbf{u}) \in \mathcal{H} \times L^{2}\left(0, T ; \mathbf{L}^{2}(\Omega)\right)$. Therefore, if problem (1.1) has a strong solution $\overline{\mathbf{y}}$ for a given control $\overline{\mathbf{u}}$, then $F(\overline{\mathbf{y}}, \overline{\mathbf{u}})=(0,0)$, and applying the implicit function theorem we deduce the existence of an open neighborhood $\mathcal{A}_{0} \subset$ $L^{2}\left(0, T ; \mathbf{L}^{2}(\Omega)\right)$ of $\overline{\mathbf{u}}$ and a mapping $G: \mathcal{A}_{0} \longrightarrow \mathcal{H}$ of class $C^{\infty}$ such that $F(G(\mathbf{u}), \mathbf{u})=$ $(0,0)$ for every $\mathbf{u} \in \mathcal{A}_{0}$. The rest of the proof is immediate.

Remark 2.4. As a consequence of Corollary 2.2, we deduce that the set of controls $\mathbf{u} \in L^{2}\left(0, T ; \mathbf{L}^{2}(\Omega)\right)$ for which there exists a strong solution $\mathbf{y}_{\mathbf{u}}$ is open. Hereafter, this set will be denoted by $\mathcal{A}$. It is known that $\mathcal{A}$ is dense in $L^{2}\left(0, T ; \mathbf{L}^{2}(\Omega)\right)$ with 
respect to the norm of $L^{s}\left(0, T ; \mathbf{L}^{q}(\Omega)\right)$ for $s, q \in(1,+\infty)$ such that $4<\frac{2}{s}+\frac{3}{q}$; see [18]. In particular we have that for any $\mathbf{u} \in L^{2}\left(0, T ; \mathbf{L}^{2}(\Omega)\right)$ and any $\varepsilon>0$, there exists $\mathbf{v}_{\varepsilon} \in L^{2}\left(0, T ; \mathbf{L}^{2}(\Omega)\right)$ with $\left\|\mathbf{v}_{\varepsilon}\right\|_{L^{1}\left(0, T ; \mathbf{L}^{1}(\Omega)\right)}<\varepsilon$ such that $\mathbf{u}+\mathbf{v}_{\varepsilon} \in \mathcal{A}$.

Remark 2.5. We have seen that (1.1) admits the variational formulation given in (2.1). Analogously, (2.6) and (2.7) can be formulated in a variational form as follows: $\forall \psi \in \mathbf{Y}$,

$$
\left\{\begin{array}{l}
\left(\mathbf{z}_{\mathbf{v} t}, \boldsymbol{\psi}\right)+a\left(\mathbf{z}_{\mathbf{v}}, \boldsymbol{\psi}\right)+c\left(\mathbf{z}_{\mathbf{v}}, \mathbf{y}_{\mathbf{u}}, \boldsymbol{\psi}\right)+c\left(\mathbf{y}_{\mathbf{u}}, \mathbf{z}_{\mathbf{v}}, \boldsymbol{\psi}\right)=(\mathbf{v}, \boldsymbol{\psi}) \text { a.e. in }[0, T] \\
\mathbf{z}_{\mathbf{v}}(0)=0
\end{array}\right.
$$

$$
\left\{\begin{aligned}
\left(\mathbf{z}_{\mathbf{v}_{1} \mathbf{v}_{2} t}, \boldsymbol{\psi}\right) & +a\left(\mathbf{z}_{\mathbf{v}_{1} \mathbf{v}_{2}}, \boldsymbol{\psi}\right)+c\left(\mathbf{z}_{\mathbf{v}_{1} \mathbf{v}_{2}}, \mathbf{y}_{\mathbf{u}}, \boldsymbol{\psi}\right)+c\left(\mathbf{y}_{\mathbf{u}}, \mathbf{z}_{\mathbf{v}_{1} \mathbf{v}_{2}}, \boldsymbol{\psi}\right) \\
& +c\left(\mathbf{z}_{\mathbf{v}_{1}}, \mathbf{z}_{\mathbf{v}_{2}}, \boldsymbol{\psi}\right)+c\left(\mathbf{z}_{\mathbf{v}_{2}}, \mathbf{z}_{\mathbf{v}_{1}}, \boldsymbol{\psi}\right)=0 \text { a.e.in }[0, T] \\
\mathbf{z}_{\mathbf{v}_{1} \mathbf{v}_{2}}(0)= & 0
\end{aligned}\right.
$$

Remark 2.6. The use of $\mathbf{P}_{\mathbf{H}}$ in the definition of $F$ given in the proof of Corollary 2.3 is necessary. In principle, one could consider the mapping

$$
\begin{aligned}
& F: \mathbf{H}^{2,1}\left(\Omega_{T}\right) \cap C([0, T], \mathbf{Y}) \times L^{2}\left(0, T ; \mathbf{L}^{2}(\Omega)\right) \longrightarrow L^{2}\left(0, T ; \mathbf{L}^{2}(\Omega)\right) \times \mathbf{Y}, \\
& F(\mathbf{y}, \mathbf{u})=\left(\frac{\partial \mathbf{y}}{\partial t}-\nu \Delta \mathbf{y}+(\mathbf{y} \cdot \nabla) \mathbf{y}-(\mathbf{f}+\mathbf{u}), \mathbf{y}(0)-\mathbf{y}_{0}\right) .
\end{aligned}
$$

Then, we have that $F$ is of class $C^{\infty}$ and

$$
\frac{\partial F}{\partial \mathbf{y}}(\mathbf{y}, \mathbf{u}) \mathbf{z}=\left(\frac{\partial \mathbf{z}}{\partial t}+-\nu \Delta \mathbf{z}+(\mathbf{y} \cdot \nabla) \mathbf{z}+(\mathbf{z} \cdot \nabla) \mathbf{y}, \mathbf{z}(0)\right)
$$

Again, the identity $\frac{\partial F}{\partial \mathbf{y}}(\mathbf{y}, \mathbf{u}) \mathbf{z}=\left(\mathbf{v}, \mathbf{z}_{0}\right) \in L^{2}\left(0, T ; \mathbf{L}^{2}(\Omega)\right) \times \mathbf{Y}$ is equivalent to

$$
\left\{\begin{array}{l}
\left(\mathbf{z}_{t}, \boldsymbol{\psi}\right)+a(\mathbf{z}, \boldsymbol{\psi})+c(\mathbf{z}, \mathbf{y}, \boldsymbol{\psi})+c(\mathbf{y}, \mathbf{z}, \boldsymbol{\psi})=(\mathbf{v}, \boldsymbol{\psi}) \forall \boldsymbol{\psi} \in \mathbf{Y} \\
\mathbf{z}(0)=\mathbf{z}_{0}
\end{array}\right.
$$

However, $\frac{\partial F}{\partial \mathbf{y}}(\mathbf{y}, \mathbf{u})$ is not an isomorphism. Indeed, observe that $\mathbf{v}$ and $\mathbf{P}_{\mathbf{H}} \mathbf{v}$ lead to the same solution $\mathbf{y}$ of the above system because $(\mathbf{v}, \boldsymbol{\psi})=\left(\mathbf{P}_{\mathbf{H}} \mathbf{v}, \boldsymbol{\psi}\right)$ for every $\boldsymbol{\psi} \in \mathbf{Y}$. This situation is avoided by introducing the projection $\mathbf{P}_{\mathbf{H}}$ in the definition of $F$. It is enough to observe that if $\mathbf{u}, \mathbf{v} \in L^{2}(0, T ; \mathbf{H})$ and $(\mathbf{v}, \boldsymbol{\psi})=(\mathbf{u}, \boldsymbol{\psi})$ for every $\boldsymbol{\psi} \in \mathbf{Y}$, then $\mathbf{u}=\mathbf{v}$. The reader is referred to [21, Chap. 1] for details.

3. The control problem. In this section, we define in a precise way the optimal control problem, we prove the existence of at least one solution, and we derive the first and second order optimality conditions. First, we define the set of admissible controls as follows: $\mathcal{U}_{a d}=\mathcal{A} \cap \mathcal{U}_{\alpha, \beta}$, with

$$
\mathcal{U}_{\alpha, \beta}=\left\{\mathbf{u} \in L^{2}\left(0, T ; \mathbf{L}^{2}(\Omega)\right): \alpha_{j} \leq u_{j}(t, x) \leq \beta_{j} \text { for a.a. }(t, x) \in \Omega_{T}, 1 \leq j \leq 3\right\},
$$

where $-\infty \leq \alpha_{j}<\beta_{j} \leq+\infty$ for $1 \leq j \leq 3$, and $\mathcal{A}$ is defined in Remark 2.4. In what follows, we will make the following assumption:

$$
\mathcal{U}_{a d} \neq \emptyset \text {. }
$$

Now, we consider the functional $J: \mathcal{A} \longrightarrow \mathbb{R}$ defined by

$$
\begin{aligned}
J(\mathbf{u})= & \frac{1}{8} \int_{0}^{T}\left\|\mathbf{y}_{\mathbf{u}}(t)-\mathbf{y}_{d}(t)\right\|_{\mathbf{L}^{4}(\Omega)}^{8} d t \\
& +\frac{\gamma}{2} \int_{\Omega}\left|\mathbf{y}_{\mathbf{u}}(T, x)-\mathbf{y}_{\Omega}(x)\right|^{2} d x+\frac{\lambda}{2} \int_{0}^{T} \int_{\Omega}|\mathbf{u}(t, x)|^{2} d x d t,
\end{aligned}
$$

Copyright $@$ by SIAM. Unauthorized reproduction of this article is prohibited. 
where $\mathbf{y}_{\mathbf{u}}=G(\mathbf{u})$ is the state associated to $\mathbf{u}$, the target $\mathbf{y}_{d} \in L^{14}\left(0, T ; \mathbf{L}^{6}(\Omega)\right)$, $\mathbf{y}_{\Omega} \in \mathbf{H}_{0}^{1}(\Omega), \gamma \geq 0$, and $\lambda>0$. It is obvious that $J(\mathbf{u})=\mathcal{J}\left(\mathbf{u}, \mathbf{y}_{\mathbf{u}}\right) \forall \mathbf{u} \in \mathcal{A}$. The regularity assumed for $\mathbf{y}_{d}$ is needed in the proof of Theorem 3.1. Finally, we define the control problem

$$
\text { (P) }\left\{\begin{array}{l}
\min J(\mathbf{u}) \\
\mathbf{u} \in \mathcal{U}_{a d}
\end{array}\right.
$$

As an immediate consequence of Corollary 2.3 we have the following differentiability properties of $J$.

Theorem 3.1. The cost functional $J: \mathcal{A} \longrightarrow \mathbb{R}$ is of class $C^{\infty}$, and for every $\mathbf{u} \in \mathcal{A}$ and $\mathbf{v} \in L^{2}\left(0, T ; \mathbf{L}^{2}(\Omega)\right)$ we have

$$
\begin{aligned}
J^{\prime}(\mathbf{u}) \mathbf{v} & =\int_{0}^{T} \int_{\Omega}\left(\boldsymbol{\varphi}_{\mathbf{u}}+\lambda \mathbf{u}\right) \mathbf{v} d x d t \\
J^{\prime \prime}(\mathbf{u}) \mathbf{v}^{2} & =4 \int_{0}^{T}\left(\int_{\Omega}\left|\mathbf{y}_{\mathbf{u}}-\mathbf{y}_{d}\right|^{2}\left(\mathbf{y}_{\mathbf{u}}-\mathbf{y}_{d}\right) \mathbf{z}_{\mathbf{v}} d x\right)^{2} d t \\
& +3 \int_{0}^{T}\left\|\mathbf{y}_{\mathbf{u}}-\mathbf{y}_{d}\right\|_{\mathbf{L}^{4}(\Omega)}^{4}\left(\int_{\Omega}\left|\mathbf{y}_{\mathbf{u}}-\mathbf{y}_{d}\right|^{2}\left|\mathbf{z}_{\mathbf{v}}\right|^{2} d x\right) d t \\
& -2 \int_{0}^{T} \int_{\Omega}\left(\mathbf{z}_{\mathbf{v}} \cdot \nabla\right) \mathbf{z}_{\mathbf{v}} \varphi_{\mathbf{u}} d x d t+\lambda \int_{0}^{T} \int_{\Omega}|\mathbf{v}|^{2} d x d t
\end{aligned}
$$

where $\mathbf{z}_{\mathbf{v}}=G^{\prime}(\mathbf{u}) \mathbf{v}$ is the solution of (2.6) and $\boldsymbol{\varphi}_{\mathbf{u}} \in \mathbf{H}^{2,1}\left(\Omega_{T}\right) \cap C([0, T], \mathbf{Y})$ is the unique element satisfying

$$
\left\{\begin{aligned}
&-\boldsymbol{\varphi}_{\mathbf{u} t}-\nu \Delta \boldsymbol{\varphi}_{\mathbf{u}}-\left(\mathbf{y}_{\mathbf{u}} \cdot \nabla\right) \boldsymbol{\varphi}_{\mathbf{u}}+\left(\nabla \mathbf{y}_{\mathbf{u}}\right)^{T} \boldsymbol{\varphi}_{\mathbf{u}}+\nabla \pi \\
& \quad=\left\|\mathbf{y}_{\mathbf{u}}-\mathbf{y}_{d}\right\|_{\mathbf{L}^{4}(\Omega)}^{4}\left|\mathbf{y}_{\mathbf{u}}-\mathbf{y}_{d}\right|^{2}\left(\mathbf{y}_{\mathbf{u}}-\mathbf{y}_{d}\right) \quad \text { in } \Omega_{T} \\
& \operatorname{div} \boldsymbol{\varphi}_{\mathbf{u}}=0 \quad \text { in } \Omega_{T}, \quad \boldsymbol{\varphi}_{\mathbf{u}}(T)=\gamma\left(\mathbf{y}_{\mathbf{u}}(T)-\mathbf{y}_{\Omega}\right) \quad \text { in } \Omega, \quad \boldsymbol{\varphi}_{\mathbf{u}}=0 \quad \text { on } \Sigma_{T}
\end{aligned}\right.
$$

with $\pi \in L^{2}\left(0, T ; H^{1}(\Omega)\right)$ uniquely defined up to the addition of a function of $L^{2}(0, T)$.

Observe that the assumption on $\mathbf{y}_{d}$ and the regularity $\mathbf{y}_{\mathbf{u}} \in \mathbf{H}^{2,1}\left(\Omega_{T}\right)$ imply that

$$
\left\|\mathbf{y}_{\mathbf{u}}-\mathbf{y}_{d}\right\|_{\mathbf{L}^{4}(\Omega)}^{4}\left|\mathbf{y}_{\mathbf{u}}-\mathbf{y}_{d}\right|^{2}\left(\mathbf{y}_{\mathbf{u}}-\mathbf{y}_{d}\right) \in L^{2}\left(0, T ; \mathbf{L}^{2}(\Omega)\right),
$$

and hence Theorem 2.1 shows that $\varphi_{\mathbf{u}} \in \mathbf{H}^{2,1}\left(\Omega_{T}\right)$.

The variational formulation of (3.5) is written as follows:

$$
\left\{\begin{aligned}
-\left(\boldsymbol{\varphi}_{\mathbf{u}, t}, \boldsymbol{\psi}\right) & +a\left(\boldsymbol{\varphi}_{\mathbf{u}}, \boldsymbol{\psi}\right)+c\left(\boldsymbol{\psi}, \mathbf{y}_{\mathbf{u}}, \boldsymbol{\varphi}_{\mathbf{u}}\right)+c\left(\mathbf{y}_{\mathbf{u}}, \boldsymbol{\psi}, \boldsymbol{\varphi}_{\mathbf{u}}\right) \\
& =\left\|\mathbf{y}_{\mathbf{u}}-\mathbf{y}_{d}\right\|_{\mathbf{L}^{4}(\Omega)}^{4}\left(\left|\mathbf{y}-\mathbf{y}_{d}\right|^{2}\left(\mathbf{y}-\mathbf{y}_{d}\right), \boldsymbol{\psi}\right) \quad \forall \boldsymbol{\psi} \in \mathbf{Y} \\
\boldsymbol{\varphi}_{\mathbf{u}}(T)=\gamma\left(\mathbf{y}_{\mathbf{u}}(T)-\mathbf{y}_{\Omega}\right) &
\end{aligned}\right.
$$

The next theorem establishes the existence of at least one solution for $(\mathrm{P})$, as well as the first order optimality conditions satisfied by any local minimum of $(\mathrm{P})$.

TheOREM 3.2. Under assumption (3.1), (P) has at least one solution. Moreover, for any local solution $\overline{\mathbf{u}}$, there exist $\overline{\mathbf{y}}, \overline{\boldsymbol{\varphi}} \in \mathbf{H}^{2,1}\left(\Omega_{T}\right) \cap C([0, T], \mathbf{Y})$ and $\bar{p}, \bar{\pi} \in$ 
$L^{2}\left(0, T ; H^{1}(\Omega)\right)$ such that

$$
\begin{aligned}
& \left\{\begin{array}{c}
\overline{\mathbf{y}}_{t}-\nu \Delta \overline{\mathbf{y}}+(\overline{\mathbf{y}} \cdot \nabla) \overline{\mathbf{y}}+\nabla \bar{p}=\mathbf{f}+\overline{\mathbf{u}} \quad \text { in } \Omega_{T}, \\
\operatorname{div} \overline{\mathbf{y}}=0 \quad \text { in } \Omega_{T}, \quad \overline{\mathbf{y}}(0)=\overline{\mathbf{y}}_{0} \quad \text { in } \Omega, \quad \overline{\mathbf{y}}=0 \quad \text { on } \Sigma_{T},
\end{array}\right. \\
& \left\{\begin{array}{c}
-\overline{\boldsymbol{\varphi}}_{t}-\nu \Delta \overline{\boldsymbol{\varphi}}-(\overline{\mathbf{y}} \cdot \nabla) \overline{\boldsymbol{\varphi}}+(\nabla \overline{\mathbf{y}})^{T} \overline{\boldsymbol{\varphi}}+\nabla \pi \\
=\left\|\overline{\mathbf{y}}-\mathbf{y}_{d}\right\|_{\mathbf{L}^{4}(\Omega)}^{4}\left|\overline{\mathbf{y}}-\mathbf{y}_{d}\right|^{2}\left(\overline{\mathbf{y}}-\mathbf{y}_{d}\right) \quad \text { in } \Omega_{T}, \\
\operatorname{div} \bar{\varphi}=0 \quad \text { in } \Omega_{T}, \quad \overline{\boldsymbol{\varphi}}(T)=\gamma\left(\overline{\mathbf{y}}(T)-\mathbf{y}_{\Omega}\right) \quad \text { in } \Omega, \quad \overline{\boldsymbol{\varphi}}=0 \quad \text { on } \Sigma_{T},
\end{array}\right. \\
& \int_{0}^{T} \int_{\Omega}(\overline{\boldsymbol{\varphi}}+\lambda \overline{\mathbf{u}})(\mathbf{u}-\overline{\mathbf{u}}) d x d t \geq 0 \quad \forall \mathbf{u} \in \mathcal{U}_{\alpha, \beta} .
\end{aligned}
$$

Moreover, the regularity property

$$
\overline{\mathbf{u}} \in \mathbf{H}^{1}\left(\Omega_{T}\right) \cap C\left([0, T], \mathbf{H}^{1}(\Omega)\right) \cap L^{2}\left(0, T ; \mathbf{W}^{1,6}(\Omega)\right) \cap L^{2}(0, T ; \mathbf{C}(\bar{\Omega}))
$$

holds.

Proof. Let us prove the existence of a solution. Since $\mathcal{U}_{a d}$ is nonempty, there exists a minimizing sequence $\left\{\mathbf{u}_{k}\right\}_{k=1}^{\infty} \subset \mathcal{U}_{a d}$ of (P). Let us set $\mathbf{y}_{k}=G\left(\mathbf{u}_{k}\right) \in$ $\mathbf{H}^{2,1}\left(\Omega_{T}\right) \cap C([0, T], \mathbf{Y})$. From the definition of the functional $J$ we deduce

$$
\frac{1}{8}\left\|\mathbf{y}_{k}\right\|_{L^{8}\left(0, T ; \mathbf{L}^{4}(\Omega)\right)}^{8}+\frac{\lambda}{2}\left\|\mathbf{u}_{k}\right\|_{\mathbf{L}^{2}(\Omega)}^{2} \leq J\left(\mathbf{u}_{k}\right) \leq J\left(\mathbf{u}_{1}\right)<\infty \quad \forall k .
$$

Hence, $\left\{\mathbf{u}_{k}\right\}_{k=1}^{\infty}$ and $\left\{\mathbf{y}_{k}\right\}_{k=1}^{\infty}$ are bounded in $L^{2}\left(0, T ; \mathbf{L}^{2}(\Omega)\right)$ and $L^{8}\left(0, T ; \mathbf{L}^{4}(\Omega)\right)$, respectively. By taking subsequences, if necessary, we can assume that $\mathbf{u}_{k} \rightarrow \overline{\mathbf{u}}$ and $\mathbf{y}_{k} \rightarrow \overline{\mathbf{y}}$ weakly in $L^{2}\left(0, T ; \mathbf{L}^{2}(\Omega)\right)$ and $L^{8}\left(0, T ; \mathbf{L}^{4}(\Omega)\right)$, respectively. From (2.5), we deduce that $\mathbf{y}_{k} \rightarrow \overline{\mathbf{y}}$ weakly in $\mathbf{H}^{2,1}\left(\Omega_{T}\right)$. Using the compactness of the embedding $\mathbf{H}^{2,1}\left(\Omega_{T}\right) \subset L^{8}\left(0, T ; \mathbf{L}^{4}(\Omega)\right)$, it is easy to pass to the limit in the state equation and to deduce that $\overline{\mathbf{y}}$ is a strong solution of (1.1) with some pressure $\bar{p} \in L^{2}\left(0, T ; H^{1}(\Omega)\right)$. Hence, we have that $\overline{\mathbf{u}} \in \mathcal{A}$. Moreover, it is immediate that $\overline{\mathbf{u}} \in \mathcal{U}_{\alpha, \beta}$. Therefore, $\overline{\mathbf{u}} \in \mathcal{U}_{a d}$ and

$$
J(\overline{\mathbf{u}})=\lim _{k \rightarrow \infty} J\left(\mathbf{u}_{k}\right)=\inf (\mathrm{P}) .
$$

The optimality system (3.7)-(3.9) can be proved in the standard way by using the expression of $J^{\prime}$ given in (3.3). Finally, the regularity of $\overline{\mathbf{u}}$ is a consequence of the embedding $\bar{\varphi} \in \mathbf{H}^{2,1}\left(\Omega_{T}\right) \subset \mathbf{H}^{1}\left(\Omega_{T}\right) \cap C\left([0, T], \mathbf{H}^{1}(\Omega)\right) \cap L^{2}\left(0, T ; \mathbf{W}^{1,6}(\Omega)\right)$ and the projection formula

$$
\bar{u}_{j}(t, x)=\operatorname{Proj}_{\left[\alpha_{j}, \beta_{j}\right]}\left(-\frac{1}{\lambda} \bar{\varphi}_{j}(t, x)\right) \text { for a.a. }(t, x) \in \Omega_{T}, \quad 1 \leq j \leq 3,
$$

which follows from (3.9).

Now, we carry out the second order analysis of $(\mathrm{P})$. Since this control problem is not convex, some second order conditions are required for the numerical analysis of $(\mathrm{P})$. To write the second order conditions, we need to define the cone of critical directions. To this end, let us introduce the function

$$
\overline{\mathbf{d}}=\bar{\varphi}+\lambda \overline{\mathbf{u}} .
$$

Copyright (c) by SIAM. Unauthorized reproduction of this article is prohibited. 
Now we set

$$
\begin{aligned}
& \mathcal{C}_{\overline{\mathbf{u}}}=\left\{\mathbf{v} \in L^{2}\left(0, T ; \mathbf{L}^{2}(\Omega)\right): \mathbf{v} \text { satisfies }(3.13)-(3.15)\right\}, \\
& v_{j}(t, x) \geq 0 \text { if }-\infty<\alpha_{j}=\bar{u}_{j}(t, x), \\
& v_{j}(t, x) \leq 0 \text { if } \bar{u}_{j}(t, x)=\beta_{j}<+\infty, \quad j=1,2,3, \\
& v_{j}(t, x)=0 \text { if } \bar{d}_{j}(t, x) \neq 0 .
\end{aligned}
$$

Let us notice that

$$
\begin{aligned}
& J^{\prime}(\overline{\mathbf{u}}) \mathbf{v}=\int_{0}^{T} \int_{\Omega} \overline{\mathbf{d}}(t, x) \cdot \mathbf{v}(t, x) \mathrm{d} x \mathrm{~d} t, \\
& \overline{\mathbf{d}}(t, x) \cdot \mathbf{v}(t, x)=0 \text { for a.a. }(t, x) \in \Omega_{T} \text { and } \forall \mathbf{v} \in \mathcal{C}_{\overline{\mathbf{u}}} .
\end{aligned}
$$

We also deduce as usual from (3.9), for almost all $(t, x) \in \Omega_{T}$ and $j=1,2,3$,

$$
\left\{\begin{array} { c l } 
{ \overline { u } _ { j } ( t , x ) = \alpha _ { j } } & { \Rightarrow \overline { d } _ { j } ( t , x ) \geq 0 , } \\
{ \overline { u } _ { j } ( t , x ) = \beta _ { j } } & { \Rightarrow \overline { d } _ { j } ( t , x ) \leq 0 , } \\
{ \alpha _ { j } < \overline { u } _ { j } ( t , x ) < \beta _ { j } } & { \Rightarrow \overline { d } _ { j } ( t , x ) = 0 , }
\end{array} \text { and } \quad \left\{\begin{array}{rl}
\bar{d}_{j}(t, x)>0 \Rightarrow \bar{u}_{j}(t, x)=\alpha_{j}, \\
\bar{d}_{j}(t, x)<0 \Rightarrow \bar{u}_{j}(t, x)=\beta_{j} .
\end{array}\right.\right.
$$

As for the 2D flows, we have the following second order necessary and sufficient conditions; see [5].

THEOREM 3.3. Let $\overline{\mathbf{u}}$ be a local solution of problem $(\mathrm{P})$; then $J^{\prime \prime}(\overline{\mathbf{u}}) \mathbf{v}^{2} \geq 0$ $\forall \mathbf{v} \in \mathcal{C}_{\overline{\mathbf{u}}}$. Conversely, let us assume that $\overline{\mathbf{u}} \in \mathcal{U}_{\text {ad }}$ satisfies

$$
\begin{aligned}
& J^{\prime}(\overline{\mathbf{u}})(\mathbf{u}-\overline{\mathbf{u}}) \geq 0 \quad \forall \mathbf{u} \in \mathcal{U}_{\alpha, \beta}, \\
& J^{\prime \prime}(\overline{\mathbf{u}}) \mathbf{v}^{2}>0 \quad \forall \mathbf{v} \in \mathcal{C}_{\overline{\mathbf{u}}} \backslash\{0\},
\end{aligned}
$$

then there exist $\varepsilon>0$ and $\delta>0$ such that

$$
J(\overline{\mathbf{u}})+\frac{\delta}{2}\|\mathbf{u}-\overline{\mathbf{u}}\|_{L^{2}\left(0, T ; \mathbf{L}^{2}(\Omega)\right)}^{2} \leq J(\mathbf{u}) \quad \forall \mathbf{u} \in \mathcal{U}_{\alpha, \beta} \cap B_{\varepsilon}(\overline{\mathbf{u}}),
$$

where $B_{\varepsilon}(\overline{\mathbf{u}})$ is the $L^{2}\left(0, T ; \mathbf{L}^{2}(\Omega)\right)$-ball of center $\overline{\mathbf{u}}$ and radius $\varepsilon$.

4. Approximation of the control problem (P). In this section, $\Omega$ is assumed to be convex. We consider a family of triangulations $\left\{\mathcal{K}_{h}\right\}_{h>0}$ of $\bar{\Omega}$, defined in the standard way. To each element $K \in \mathcal{K}_{h}$, typically a tetrahedron or a hexahedron, we associate two parameters $h_{K}$ and $\varrho_{K}$, where $h_{K}$ denotes the diameter of the set $K$ and $\varrho_{K}$ is the diameter of the largest ball contained in $K$. Define the size of the mesh by $h=\max _{K \in \mathcal{K}_{h}} h_{K}$. We also assume that the following standard regularity assumptions on the triangulation hold:

(i) There exist two positive constants $\varrho_{\mathcal{K}}$ and $\delta_{\mathcal{K}}$ such that $\frac{h_{K}}{\varrho_{K}} \leq \varrho_{\mathcal{K}}$ and $\frac{h}{h_{K}} \leq \delta_{\mathcal{K}}$ $\forall K \in \mathcal{K}_{h}$ and $\forall h>0$.

(ii) Define $\bar{\Omega}_{h}=\cup_{K \in \mathcal{K}_{h}} K$, and let $\Omega_{h}$ and $\Gamma_{h}$ denote its interior and its boundary, respectively. We assume that the vertices of $\mathcal{K}_{h}$ placed on the boundary $\Gamma_{h}$ are points of $\Gamma$.

Since $\Omega$ is convex, from the last assumption we have that $\Omega_{h}$ is also convex. Moreover, we assume that

$$
\left|\Omega \backslash \Omega_{h}\right| \leq C h^{2} \quad \text { and } \quad \mathbf{y}_{d} \in L^{\infty}\left(0, T ; \mathbf{L}^{6}(\Omega)\right) \cap L^{2}\left(0, T ; \mathbf{L}^{\infty}(\Omega)\right) .
$$

Copyright $@$ by SIAM. Unauthorized reproduction of this article is prohibited. 
On the mesh $\mathcal{K}_{h}$ we consider two finite-dimensional spaces $\mathbf{Z}_{h} \subset \mathbf{H}_{0}^{1}(\Omega)$ and $Q_{h} \subset L_{0}^{2}(\Omega)$ formed by piecewise polynomials in $\Omega_{h}$ and vanishing in $\Omega \backslash \Omega_{h}$. We make the following assumptions on these spaces.

(A1) If $\mathbf{z} \in \mathbf{H}^{1+l}(\Omega) \cap \mathbf{H}_{0}^{1}(\Omega)$, then

$$
\inf _{\mathbf{z}_{h} \in \mathbf{Z}_{h}}\left\|\mathbf{z}-\mathbf{z}_{h}\right\|_{\mathbf{H}^{s}\left(\Omega_{h}\right)} \leq C h^{l+1-s}\|\mathbf{z}\|_{\mathbf{H}^{1+l}(\Omega)} \text { for } 0 \leq l \leq 1 \text { and } s=0,1 .
$$

(A2) If $q \in H^{1}(\Omega) \cap L_{0}^{2}(\Omega)$, then

$$
\inf _{q_{h} \in Q_{h}}\left\|q-q_{h}\right\|_{L^{2}\left(\Omega_{h}\right)} \leq C h\|q\|_{H^{1}(\Omega)} .
$$

(A3) The subspaces $\mathbf{Z}_{h}$ and $Q_{h}$ satisfy the following inf-sup condition: $\exists c>0$ such that

$$
\inf _{q_{h} \in Q_{h}} \sup _{\mathbf{z}_{h} \in \mathbf{Z}_{h}} \frac{b\left(\mathbf{z}_{h}, q_{h}\right)}{\left\|\mathbf{z}_{h}\right\|_{\mathbf{H}^{1}\left(\Omega_{h}\right)}\left\|q_{h}\right\|_{L^{2}\left(\Omega_{h}\right)}} \geq c,
$$

where $b: \mathbf{H}^{1}(\Omega) \times L^{2}(\Omega) \longrightarrow \mathbb{R}$ is defined by

$$
b(\mathbf{z}, q)=\int_{\Omega} q(x) \operatorname{div} \mathbf{z}(x) \mathrm{d} x .
$$

These assumptions are satisfied by the usual finite elements considered in the discretization of Navier-Stokes equations; see [10, Chap. 2].

We also consider a subspace $\mathbf{Y}_{h}$ of $\mathbf{Z}_{h}$ defined by

$$
\mathbf{Y}_{h}=\left\{\mathbf{y}_{h} \in \mathbf{Z}_{h}: b\left(\mathbf{y}_{h}, q_{h}\right)=0 \forall q_{h} \in Q_{h}\right\},
$$

and we set

$$
\mathbf{U}_{h}=\left\{\mathbf{u}_{h} \in \mathbf{L}^{2}\left(\Omega_{h}\right): \mathbf{u}_{\left.h\right|_{K}} \equiv \mathbf{u}_{K} \in \mathbb{R}^{3} \forall K \in \mathcal{K}_{h}\right\} .
$$

It is well known that, under the previous assumptions, given an element $\mathbf{z} \in$ $\mathbf{H}^{2}(\Omega) \cap \mathbf{Y}$, there exist elements $\mathbf{z}_{h} \in \mathbf{Y}_{h}$ such that

$$
\left\|\mathbf{z}-\mathbf{z}_{h}\right\|_{\mathbf{H}_{0}^{1}(\Omega)} \leq C h\|\mathbf{z}\|_{\mathbf{H}^{2}(\Omega)},
$$

where $C$ is independent of $h$ and $\mathbf{z}$. Moreover, from this estimate and an inverse inequality it is easy to prove that for the usual finite elements considered in the discretization of Navier-Stokes equations, the following estimate holds:

$$
\lim _{h \rightarrow 0}\left\|\mathbf{z}-\mathbf{z}_{h}\right\|_{\mathbf{W}^{1,4}\left(\Omega_{h}\right)}=0 .
$$

Hence, in addition to assumptions (A1)-(A3), we will assume

$$
\lim _{h \rightarrow 0} \inf _{\mathbf{z}_{h} \in \mathbf{Y}_{h}}\left\|\mathbf{z}-\mathbf{z}_{h}\right\|_{\mathbf{W}^{1,4}\left(\Omega_{h}\right)}=0 \quad \forall \mathbf{z} \in \mathbf{H}^{2}(\Omega) \cap \mathbf{Y} .
$$

We proceed now with the discretization in time. Let us consider a grid of points $0=t_{0}<t_{1}<\cdots<t_{N_{\tau}}=T$. We denote $\tau_{n}=t_{n}-t_{n-1}$. We make the following assumption:

$$
\exists \varrho_{0}>0 \text { such that } \tau=\max _{1 \leq n \leq N_{\tau}} \tau_{n} \leq \varrho_{0} \tau_{n} \quad \forall 1 \leq n \leq N_{\tau} \text { and } \forall \tau>0 .
$$

Copyright $@$ by SIAM. Unauthorized reproduction of this article is prohibited. 
Given a triangulation $\mathcal{K}_{h}$ of $\Omega$ and a grid of points $\left\{t_{n}\right\}_{n=0}^{N_{\tau}}$ of $[0, T]$, we set $\sigma=(\tau, h)$ and $|\sigma|=\tau+h$. Finally, we consider the following spaces:

$$
\begin{aligned}
& \mathcal{Y}_{\sigma}=\left\{\mathbf{y}_{\sigma} \in L^{2}\left(0, T ; \mathbf{Y}_{h}\right): \mathbf{y}_{\left.\sigma\right|_{\left(t_{n-1}, t_{n}\right)}} \in \mathbf{Y}_{h} \text { for } 1 \leq n \leq N_{\tau}\right\}, \\
& \mathcal{Q}_{\sigma}=\left\{q_{\sigma} \in L^{2}\left(0, T ; Q_{h}\right): q_{\left.\right|_{\left(t_{n-1}, t_{n}\right)}} \in Q_{h} \text { for } 1 \leq n \leq N_{\tau}\right\}, \\
& \mathcal{U}_{\sigma}=\left\{\mathbf{u}_{\sigma} \in L^{2}\left(0, T ; \mathbf{U}_{h}\right): \mathbf{u}_{\left.\sigma\right|_{\left(t_{n-1}, t_{n}\right)}} \in \mathbf{U}_{h} \text { for } 1 \leq n \leq N_{\tau}\right\} .
\end{aligned}
$$

We have that the functions of $\mathcal{Y}_{\sigma}, \mathcal{U}_{\sigma}$, and $\mathcal{Q}_{\sigma}$ are piecewise constant in time. We will look for the discrete controls in the space $\mathcal{U}_{\sigma}$. An element of this space can be written in the form

$$
\mathbf{u}_{\sigma}=\sum_{n=1}^{N_{\tau}} \sum_{K \in \mathcal{K}_{h}} \mathbf{u}_{n, K} \chi_{n} \chi_{K}, \text { with } \mathbf{u}_{n, K} \in \mathbb{R}^{3},
$$

where $\chi_{n}$ and $\chi_{K}$ are the characteristic functions of $\left(t_{n-1}, t_{n}\right)$ and $K$, respectively. Therefore, the dimension of $\mathcal{U}_{\sigma}$ is $3 N_{\tau} N_{h}$, where $N_{h}$ is the number of elements in $\mathcal{K}_{h}$. In $\mathcal{U}_{\sigma}$ we consider the convex subset

$$
\mathcal{U}_{\sigma, a d}=\mathcal{U}_{\sigma} \cap \mathcal{U}_{\alpha, \beta}=\left\{\mathbf{u}_{\sigma} \in \mathcal{U}_{\sigma}: \mathbf{u}_{n, K} \in \prod_{i=1}^{3}\left[\alpha_{i}, \beta_{i}\right]\right\}
$$

On the other hand, the elements of $\mathcal{Y}_{\sigma}$ can be written in the form

$$
\mathbf{y}_{\sigma}=\sum_{n=1}^{N_{\tau}} \mathbf{y}_{n, h} \chi_{n}, \quad \text { with } \mathbf{y}_{n, h} \in \mathbf{Y}_{h}
$$

where $\chi_{n}$ is as above. For every discrete state $\mathbf{y}_{\sigma}$, we will fix $\mathbf{y}_{\sigma}\left(t_{n}\right)=\mathbf{y}_{n, h}$ so that $\mathbf{y}_{\sigma}$ is continuous on the left. In particular, we have $\mathbf{y}_{\sigma}(T)=\mathbf{y}_{\sigma}\left(t_{N_{\tau}}\right)=y_{N_{\tau}, h}$.

To define the discrete control problem, we have to consider the numerical discretization of the state equation (1.1) or, equivalently, (2.1). We achieve this goal by using a discontinuous time-stepping Galerkin method, with piecewise constants in time and conforming finite element spaces in space. For any $\mathbf{u} \in L^{2}\left(0, T ; \mathbf{L}^{2}(\Omega)\right)$, the discrete state equation is given by

$$
\left\{\begin{array}{l}
\text { for } n=1, \ldots, N_{\tau}, \text { and } \forall \boldsymbol{\psi}_{h} \in \mathbf{Y}_{h} \\
\left(\frac{\mathbf{y}_{n, h}-\mathbf{y}_{n-1, h}}{\tau_{n}}, \boldsymbol{\psi}_{h}\right)+a\left(\mathbf{y}_{n, h}, \boldsymbol{\psi}_{h}\right)+c\left(\mathbf{y}_{n, h}, \mathbf{y}_{n, h}, \boldsymbol{\psi}_{h}\right)=\left(\mathbf{f}_{n}+\mathbf{u}_{n}, \boldsymbol{\psi}_{h}\right) \\
\mathbf{y}_{0, h}=\mathbf{y}_{0 h}
\end{array}\right.
$$

where

$$
\begin{aligned}
& \left(\mathbf{f}_{n}, \boldsymbol{\psi}_{h}\right)=\frac{1}{\tau_{n}} \int_{t_{n-1}}^{t_{n}}\left(\mathbf{f}(t), \boldsymbol{\psi}_{h}\right) \mathrm{d} t,\left(\mathbf{u}_{n}, \boldsymbol{\psi}_{h}\right)=\frac{1}{\tau_{n}} \int_{t_{n-1}}^{t_{n}}\left(\mathbf{u}(t), \boldsymbol{\psi}_{h}\right) \mathrm{d} t \\
& \mathbf{y}_{0 h} \in \mathbf{Y}_{h} \text { with }\left\|\mathbf{y}_{0}-\mathbf{y}_{0 h}\right\|_{\mathbf{L}^{2}\left(\Omega_{h}\right)} \leq C h .
\end{aligned}
$$

Applying Brouwer's theorem, one can easily prove the existence of at least one solution $\mathbf{y}_{\sigma} \in \mathcal{Y}_{\sigma}$ of (4.9) for every $\mathbf{u} \in L^{2}\left(0, T ; \mathbf{L}^{2}(\Omega)\right)$. The uniqueness is a more delicate issue. For the uniqueness we need the following extra assumption:

$$
\exists C_{0}>0 \text { such that } \tau \leq C_{0} h^{2} \forall \sigma=(\tau, h) .
$$


From [5, Cor. 4.9] we obtain the following result.

THEOREM 4.1. Under assumption (4.12), for any bounded set $\mathbf{K} \subset L^{2}\left(0, T ; \mathbf{L}^{2}(\Omega)\right) \times$ $L^{8}\left(0, T ; \mathbf{L}^{4}(\Omega)\right)$ there exists a constant $\tau_{\mathbf{K}}>0$ such that for any $\mathbf{u} \in \mathcal{A}$ with $\left(\mathbf{u}, \mathbf{y}_{\mathbf{u}}\right) \in$ $\mathbf{K}$, (4.9) has a unique solution $\mathbf{y}_{\sigma}(\mathbf{u}) \in \mathcal{Y}_{\sigma}$ for every $\tau<\tau_{\mathbf{K}}$. Moreover, the sequence $\left\{\mathbf{y}_{\sigma}(\mathbf{u})\right\}_{\tau<\tau_{K}}$ is bounded in $L^{\infty}\left(0, T ; \mathbf{H}_{0}^{1}\left(\Omega_{h}\right)\right)$. Finally, if $\mathbf{u}, \mathbf{v} \in \mathcal{A}$ and $\left(\mathbf{u}, \mathbf{y}_{\mathbf{u}}\right),\left(\mathbf{v}, \mathbf{y}_{\mathbf{v}}\right) \in \mathbf{K}$, then there exists a constant $C_{\mathbf{K}}>0$ such that

$$
\begin{aligned}
& \left\|\mathbf{y}_{\mathbf{u}}-\mathbf{y}_{\sigma}(\mathbf{v})\right\|_{L^{\infty}\left(0, T ; \mathbf{L}^{2}\left(\Omega_{h}\right)\right)}+\left\|\mathbf{y}_{\mathbf{u}}-\mathbf{y}_{\sigma}(\mathbf{v})\right\|_{L^{2}\left(0, T ; \mathbf{H}^{1}\left(\Omega_{h}\right)\right)} \\
& \leq C_{\mathbf{K}}\left\{h+\|\mathbf{u}-\mathbf{v}\|_{L^{2}\left(0, T ; \mathbf{L}^{2}(\Omega)\right)}\right\} .
\end{aligned}
$$

The proof given in [5] for the 2D case is also valid for the $3 \mathrm{D}$ case assuming that the controls belong to $\mathcal{A}$ and the pair control-state belongs to $\mathbf{K}$. It is enough to take into account the estimate (2.5).

Now we define the discrete control problem as follows:

$$
\left(\mathrm{P}_{\sigma}\right)\left\{\begin{array}{l}
\min \mathcal{J}_{\sigma}\left(\mathbf{u}_{\sigma}, \mathbf{y}_{\sigma}\right), \\
\left(\mathbf{u}_{\sigma}, \mathbf{y}_{\sigma}\right) \in \mathcal{U}_{\sigma, a d} \times \mathcal{Y}_{\sigma} \operatorname{satisfy}(4.9),
\end{array}\right.
$$

with

$$
\begin{aligned}
\mathcal{J}_{\sigma}\left(\mathbf{u}_{\sigma}, \mathbf{y}_{\sigma}\right)= & \frac{1}{2} \int_{0}^{T}\left\|\mathbf{y}_{\sigma}(t)-\mathbf{y}_{d}(t)\right\|_{\mathbf{L}^{4}\left(\Omega_{h}\right)}^{8} d t \\
& +\frac{\gamma}{2} \int_{\Omega_{h}}\left|\mathbf{y}_{\sigma}(T)-\mathbf{y}_{\Omega h}\right|^{2} d x+\frac{\lambda}{2} \int_{0}^{T} \int_{\Omega_{h}}\left|\mathbf{u}_{\sigma}\right|^{2} d x d t
\end{aligned}
$$

where $\mathbf{y}_{\Omega h} \in \mathbf{Y}_{h}$ satisfies

$$
\exists C>0 \text { such that }\left\|\mathbf{y}_{\Omega}-\mathbf{y}_{\Omega h}\right\|_{\mathbf{L}^{2}\left(\Omega_{h}\right)} \leq C h .
$$

For instance, $\mathbf{y}_{\Omega h}$ can be defined as the projection of $\mathbf{y}_{\Omega}$.

The following result is crucial in the rest of the paper.

THEOREM 4.2. Under assumption (4.12), the control problem $\left(\mathrm{P}_{\sigma}\right)$ has at least one solution $\left(\overline{\mathbf{u}}_{\sigma}, \overline{\mathbf{y}}_{\sigma}\right)$, and there exists a constant $\mu_{0}>0$ such that the set of all solutions $\left\{\left(\overline{\mathbf{u}}_{\sigma}, \overline{\mathbf{y}}_{\sigma}\right)\right\}_{|\sigma| \leq \mu_{0}}$ is bounded in $L^{2}\left(0, T ; \mathbf{L}^{2}(\Omega)\right) \times L^{8}\left(0, T ; \mathbf{L}^{4}(\Omega)\right)$. If $(\overline{\mathbf{u}}, \overline{\mathbf{y}})$ is a weak limit in $L^{2}\left(0, T ; \mathbf{L}^{2}(\Omega)\right) \times L^{8}\left(0, T ; \mathbf{L}^{4}(\Omega)\right)$ of a subsequence of solutions $\left\{\left(\overline{\mathbf{u}}_{\sigma}, \overline{\mathbf{y}}_{\sigma}\right)\right\}_{\sigma}$ with $\sigma \rightarrow 0$, then $\overline{\mathbf{u}}$ is a solution of $(\mathrm{P}), \overline{\mathbf{y}}=\overline{\mathbf{y}}_{\overline{\mathbf{u}}} \in \mathbf{H}^{2,1}\left(\Omega_{T}\right)$, and

$$
\begin{aligned}
& \lim _{\sigma \rightarrow 0}\left\|\overline{\mathbf{u}}_{\sigma}-\overline{\mathbf{u}}\right\|_{L^{2}\left(0, T ; \mathbf{L}^{2}\left(\Omega_{h}\right)\right)}=0, \\
& \lim _{\sigma \rightarrow 0}\left\{\left\|\overline{\mathbf{y}}_{\sigma}-\overline{\mathbf{y}}\right\|_{L^{8}\left(0, T ; \mathbf{L}^{4}\left(\Omega_{h}\right)\right)}+\gamma\left\|\overline{\mathbf{y}}_{\sigma}(T)-\overline{\mathbf{y}}(T)\right\|_{\mathbf{L}^{2}\left(\Omega_{h}\right)}\right\}=0 .
\end{aligned}
$$

Furthermore, $\overline{\mathbf{u}}_{\sigma} \in \mathcal{U}_{\text {ad }}$ for every $|\sigma| \leq \mu_{0}$.

Proof. Along this proof, every element of $\mathbf{u}_{\sigma} \in \mathcal{U}_{\sigma}$ will be extended to $(0, T) \times \Omega$ by setting $\mathbf{u}(t, x)=(a+b) / 2$ for almost every $(t, x) \in(0, T) \times\left(\Omega \backslash \Omega_{h}\right)$. It is obvious that the set of elements $\left(\mathbf{u}_{\sigma}, \mathbf{y}_{\sigma}\right) \in \mathcal{U}_{\sigma, a d} \times L^{8}\left(0, T ; \mathbf{L}^{4}(\Omega)\right)$ satisfying (4.9) is not empty and closed, and $\mathcal{J}_{\sigma}$ is continuous and coercive on this set, and hence $\left(\mathrm{P}_{\sigma}\right)$ has at least one solution. According to Theorem 3.2, there exists at least one solution $\hat{\mathbf{u}}$ of $(\mathrm{P})$. Let $\hat{\mathbf{u}}_{\sigma}$ be the $L^{2}\left(0, T ; \mathbf{L}^{2}\left(\Omega_{h}\right)\right)$-projection of $\hat{\mathbf{u}}$ on $\mathcal{U}_{\sigma}$. Then it is immediate that $\hat{\mathbf{u}}_{\sigma} \rightarrow \hat{\mathbf{u}}$ strongly in $L^{2}\left(0, T ; \mathbf{L}^{2}(\Omega)\right)$, and additionally $\hat{\mathbf{u}}_{\sigma} \in \mathcal{U}_{\sigma, a d}$. Consequently, we have that $\hat{\mathbf{u}}_{\sigma} \in \mathcal{A}$ if $|\sigma| \leq \mu_{1}$ for some $\mu_{1}>0$. Now, from Corollary 2.3 it follows that $\mathbf{y}_{\hat{\mathbf{u}}_{\sigma}}=G\left(\hat{\mathbf{u}}_{\sigma}\right) \rightarrow \hat{\mathbf{y}}=G(\hat{\mathbf{u}})$ in $\mathbf{H}^{2,1}\left(\Omega_{T}\right)$. Hence, if we take $\mathbf{K}=\left\{\left(\hat{\mathbf{u}}_{\sigma}, \mathbf{y}_{\hat{\mathbf{u}}_{\sigma}}\right):|\sigma| \leq \mu_{1}\right\}$, 
then Theorem 4.1 implies that, for $\mu_{1}$ small enough, (4.9) has a unique solution $\hat{\mathbf{y}}_{\sigma}=\mathbf{y}_{\sigma}\left(\hat{\mathbf{u}}_{\sigma}\right) \in \mathcal{Y}_{\sigma}$, associated with $\hat{\mathbf{u}}_{\sigma} \forall|\sigma| \leq \mu_{1}$. Moreover, $\left\{\hat{\mathbf{y}}_{\sigma}\right\}_{|\sigma| \leq \mu_{1}}$ is bounded in $L^{\infty}\left(0, T ; \mathbf{H}_{0}^{1}(\Omega)\right)$. Using this boundedness and (4.13), we infer

$$
\begin{aligned}
& \int_{0}^{T}\left\|\hat{\mathbf{y}}(t)-\hat{\mathbf{y}}_{\sigma}(t)\right\|_{\mathbf{L}^{4}(\Omega)}^{8} d t \leq C_{0} \int_{0}^{T}\left\|\hat{\mathbf{y}}(t)-\hat{\mathbf{y}}_{\sigma}(t)\right\|_{\mathbf{H}_{0}^{1}(\Omega)}^{8} \\
\leq & C_{0}\left\|\hat{\mathbf{y}}-\hat{\mathbf{y}}_{\sigma}\right\|_{L^{\infty}\left(0, T ; \mathbf{H}_{0}^{1}(\Omega)\right)}^{6} \int_{0}^{T}\left\|\hat{\mathbf{y}}(t)-\hat{\mathbf{y}}_{\sigma}(t)\right\|_{\mathbf{H}_{0}^{1}(\Omega)}^{2} \rightarrow 0 \text { as } \sigma \rightarrow 0 .
\end{aligned}
$$

In addition, there exists a constant $C$ independent of $\sigma$ such that

$$
\mathcal{J}_{\sigma}\left(\overline{\mathbf{u}}_{\sigma}, \overline{\mathbf{y}}_{\sigma}\right) \leq \mathcal{J}_{\sigma}\left(\hat{\mathbf{u}}_{\sigma}, \hat{\mathbf{y}}_{\sigma}\right) \leq C \quad \forall|\sigma| \leq \mu_{1}
$$

In what follows, we make the proof for $\gamma>0$. If $\gamma=0$, then we should remove all the convergence comments about $\overline{\mathbf{y}}_{\sigma}(T)$, and the rest remain equal. From the above inequality we obtain the boundedness of the sequence $\left\{\left(\overline{\mathbf{u}}_{\sigma}, \overline{\mathbf{y}}_{\sigma}, \overline{\mathbf{y}}_{\sigma}(T)\right)\right\}_{|\sigma| \leq \mu_{1}}$ in $L^{2}\left(0, T ; \mathbf{L}^{2}(\Omega)\right) \times L^{8}\left(0, T ; \mathbf{L}^{4}(\Omega)\right) \times \mathbf{L}^{2}(\Omega)$. By taking a subsequence if necessary, we have that $\left(\overline{\mathbf{u}}_{\sigma}, \overline{\mathbf{y}}_{\sigma}, \overline{\mathbf{y}}_{\sigma}(T)\right) \rightarrow\left(\overline{\mathbf{u}}, \overline{\mathbf{y}}, \overline{\mathbf{y}}_{T}\right)$ weakly in $L^{2}\left(0, T ; \mathbf{L}^{2}(\Omega)\right) \times L^{8}\left(0, T ; \mathbf{L}^{4}(\Omega)\right) \times$ $\mathbf{L}^{2}(\Omega)$. In addition, since $\left(\overline{\mathbf{u}}_{\sigma}, \overline{\mathbf{y}}_{\sigma}\right)$ satisfies (4.9), we also deduce, as usual, the boundedness of $\left\{\overline{\mathbf{y}}_{\sigma}\right\}_{|\sigma| \leq \mu_{1}}$ in $L^{\infty}\left(0, T ; \mathbf{L}^{2}(\Omega)\right) \cap L^{2}\left(0, T ; \mathbf{H}_{0}^{1}(\Omega)\right)$, and therefore, $\overline{\mathbf{y}}_{\sigma} \stackrel{*}{\rightarrow} \overline{\mathbf{y}}$ in $L^{\infty}\left(0, T ; \mathbf{L}^{2}(\Omega)\right)$ and $\overline{\mathbf{y}}_{\sigma} \rightarrow \overline{\mathbf{y}}$ in $L^{2}\left(0, T ; \mathbf{H}_{0}^{1}(\Omega)\right)$. Using a compactness result tailored for discontinuous Galerkin time-stepping schemes by Walkington [24, Thm. 3.1], we infer that $\overline{\mathbf{y}}_{\sigma} \rightarrow \overline{\mathbf{y}}$ strongly in $L^{2}\left(0, T ; \mathbf{L}^{2}(\Omega)\right)$. Indeed, in our case [24, Thm. 3.1] states that for any $1 \leq p, q<\infty$, if $\left\{\left\|\overline{\mathbf{y}}_{\sigma}\right\|_{L^{p}(0, T ; \mathbf{Y})}\right\}_{\sigma>0}$ and $\left\{\left\|F\left(\overline{\mathbf{y}}_{\sigma}\right)\right\|_{L^{q}\left(0, T ; \mathbf{Y}_{h}^{\prime}\right)}\right\}_{\sigma>0}$ are bounded independent of $\sigma$, with

$$
\left\langle F\left(\overline{\mathbf{y}}_{\sigma}\right), \mathbf{v}_{\sigma}\right\rangle \equiv-a\left(\overline{\mathbf{y}}_{\sigma}, \mathbf{v}_{\sigma}\right)-c\left(\overline{\mathbf{y}}_{\sigma}, \overline{\mathbf{y}}_{\sigma}, \mathbf{v}_{\sigma}\right)+\left(\overline{\mathbf{u}}_{\sigma}, \mathbf{v}_{\sigma}\right),
$$

then $\left\{\overline{\mathbf{y}}_{\sigma}\right\}_{\sigma}$ is compact in $L^{r}\left(0, T ; \mathbf{L}^{2}(\Omega)\right)$ for $1 \leq r<2 p$. Note that for $p=2$, it is easy to show that $\left\{\left\|F\left(\overline{\mathbf{y}}_{\sigma}\right)\right\|_{L^{2}\left(0, T ; \mathbf{Y}_{h}^{\prime}\right)}\right\}_{\sigma>0}$, is bounded independent of $\sigma$. We need only consider the convection terms associated to the trilinear form, and in particular,

$$
\int_{0}^{T}\left|c\left(\overline{\mathbf{y}}_{\sigma}, \overline{\mathbf{y}}_{\sigma}, \mathbf{v}_{\sigma}\right)\right| \mathrm{d} t \leq\left\|\overline{\mathbf{y}}_{\sigma}\right\|_{L^{4}\left(0, T ; L^{4}(\Omega)\right)}^{2}\left\|\mathbf{v}_{\sigma}\right\|_{L^{2}\left(0, T ; H^{1}(\Omega)\right)} \leq C\left\|\mathbf{v}_{\sigma}\right\|_{L^{2}\left(0, T ; \mathbf{Y}_{h}\right)} .
$$

As a consequence of all these convergence properties, we prove below that $\overline{\mathbf{y}} \in \mathbf{W}(0, T)$ and that $(\overline{\mathbf{u}}, \overline{\mathbf{y}})$ satisfies (2.1). Assuming that this has been already established, and taking into account that $\overline{\mathbf{y}} \in L^{8}\left(0, T ; \mathbf{L}^{4}(\Omega)\right)$, we deduce that $\overline{\mathbf{y}}$ is a strong solution of (2.1) associated with $\overline{\mathbf{u}}$, and hence $\overline{\mathbf{u}} \in \mathcal{U}_{a d}$ and $\overline{\mathbf{y}}=G(\overline{\mathbf{u}})$. It will be also established below that $\overline{\mathbf{y}}(T)=\overline{\mathbf{y}}_{T}$. Now, using the continuity and convexity of $\mathcal{J}$, along with the facts that $\left(\overline{\mathbf{u}}_{\sigma}, \overline{\mathbf{y}}_{\sigma}\right)$ and $\hat{\mathbf{u}}$ are solutions of $\left(\mathrm{P}_{\sigma}\right)$ and $(\mathrm{P})$, respectively, we get

$$
\begin{aligned}
& J(\hat{\mathbf{u}}) \leq J(\overline{\mathbf{u}})=\mathcal{J}(\overline{\mathbf{u}}, \overline{\mathbf{y}}) \leq \liminf _{\sigma \rightarrow 0} \mathcal{J}_{\sigma}\left(\overline{\mathbf{u}}_{\sigma}, \overline{\mathbf{y}}_{\sigma}\right) \\
& \quad \leq \limsup _{\sigma \rightarrow 0} \mathcal{J}_{\sigma}\left(\overline{\mathbf{u}}_{\sigma}, \overline{\mathbf{y}}_{\sigma}\right) \leq \limsup _{\sigma \rightarrow 0} \mathcal{J}_{\sigma}\left(\hat{\mathbf{u}}_{\sigma}, \hat{\mathbf{y}}_{\sigma}\right)=\mathcal{J}(\hat{\mathbf{u}}, \hat{\mathbf{y}})=J(\hat{\mathbf{u}}),
\end{aligned}
$$

which proves that

$$
\lim _{\sigma \rightarrow 0} \mathcal{J}_{\sigma}\left(\overline{\mathbf{u}}_{\sigma}, \overline{\mathbf{y}}_{\sigma}\right)=\mathcal{J}(\overline{\mathbf{u}}, \overline{\mathbf{y}})=J(\overline{\mathbf{u}})=\mathcal{J}(\hat{\mathbf{u}}, \hat{\mathbf{y}}) .
$$

Thus, $\overline{\mathbf{u}}$ is a solution of $(\mathrm{P})$. Let us prove the strong convergence $\left(\overline{\mathbf{u}}_{\sigma}, \overline{\mathbf{y}}_{\sigma}, \overline{\mathbf{y}}_{\sigma}(T)\right) \rightarrow$ $\left(\overline{\mathbf{u}}, \overline{\mathbf{y}}, \overline{\mathbf{y}}_{T}\right)$ in $L^{2}\left(0, T ; \mathbf{L}^{2}(\Omega)\right) \times L^{8}\left(0, T ; \mathbf{L}^{4}(\Omega)\right) \times \mathbf{L}^{2}(\Omega)$. For the convergence $\overline{\mathbf{u}}_{\sigma} \rightarrow \overline{\mathbf{u}}$ 
we proceed as follows:

$$
\begin{gathered}
\frac{\lambda}{2}\|\overline{\mathbf{u}}\|_{\mathbf{L}^{2}(\Omega)}^{2} \leq \liminf _{\sigma \rightarrow 0} \frac{\lambda}{2}\left\|\overline{\mathbf{u}}_{\sigma}\right\|_{\mathbf{L}^{2}(\Omega)}^{2} \leq \limsup _{\sigma \rightarrow 0} \frac{\lambda}{2}\left\|\overline{\mathbf{u}}_{\sigma}\right\|_{\mathbf{L}^{2}(\Omega)}^{2} \\
=\limsup _{\sigma \rightarrow 0}\left(\mathcal{J}_{\sigma}\left(\overline{\mathbf{u}}_{\sigma}, \overline{\mathbf{y}}_{\sigma}\right)-\left\{\frac{1}{8}\left\|\overline{\mathbf{y}}_{\sigma}-\mathbf{y}_{d}\right\|_{L^{8}\left(0, T ; \mathbf{L}^{4}(\Omega)\right)}^{8}+\frac{\gamma}{2}\left\|\overline{\mathbf{y}}_{\sigma}(T)-\mathbf{y}_{\Omega}\right\|_{\mathbf{L}^{2}(\Omega)}^{2}\right\}\right) \\
\leq \limsup _{\sigma \rightarrow 0} \mathcal{J}_{\sigma}\left(\overline{\mathbf{u}}_{\sigma}, \overline{\mathbf{y}}_{\sigma}\right)-\liminf _{\sigma \rightarrow 0}\left\{\frac{1}{8}\left\|\overline{\mathbf{y}}_{\sigma}-\mathbf{y}_{d}\right\|_{L^{8}\left(0, T ; \mathbf{L}^{4}(\Omega)\right)}^{8}+\frac{\gamma}{2}\left\|\overline{\mathbf{y}}_{\sigma}(T)-\mathbf{y}_{\Omega}\right\|_{\mathbf{L}^{2}(\Omega)}^{2}\right\} \\
\leq \mathcal{J}(\overline{\mathbf{u}}, \overline{\mathbf{y}})-\left\{\frac{1}{8}\left\|\overline{\mathbf{y}}-\mathbf{y}_{d}\right\|_{L^{8}\left(0, T ; \mathbf{L}^{4}(\Omega)\right)}^{8}+\frac{\gamma}{2}\left\|\overline{\mathbf{y}}_{T}-\mathbf{y}_{\Omega}\right\|_{\mathbf{L}^{2}(\Omega)}^{2}\right\}=\frac{\lambda}{2}\|\overline{\mathbf{u}}\|_{\mathbf{L}^{2}(\Omega)}^{2} .
\end{gathered}
$$

Hence, the uniform convexity of the space $L^{2}\left(0, T ; \mathbf{L}^{2}(\Omega)\right)$ implies the strong convergence $\overline{\mathbf{u}}_{\sigma} \rightarrow \overline{\mathbf{u}}$ in $L^{2}\left(0, T ; \mathbf{L}^{2}(\Omega)\right)$. Analogously, we prove the strong convergence of the other two terms.

Now, the strong convergence $\overline{\mathbf{u}}_{\sigma} \rightarrow \overline{\mathbf{u}}$ in $L^{2}\left(0, T ; \mathbf{L}^{2}(\Omega)\right)$ and the fact that $\overline{\mathbf{u}} \in \mathcal{A}$ imply that there exists $\mu_{0} \leq \mu_{1}$ such that $\overline{\mathbf{u}}_{\sigma} \in \mathcal{U}_{a d}$ for $|\sigma| \leq \mu_{0}$.

To conclude the proof, it remains to show that $\overline{\mathbf{y}} \in \mathbf{W}(0, T),(\overline{\mathbf{u}}, \overline{\mathbf{y}})$ satisfies (2.1), and $\overline{\mathbf{y}}(T)=\overline{\mathbf{y}}_{T}$. First, we observe that $\overline{\mathbf{y}}_{\sigma} \in L^{2}\left(0, T ; \mathbf{Y}_{h}\right)$ and $\overline{\mathbf{y}}_{\sigma} \rightarrow \overline{\mathbf{y}}$ in $L^{2}\left(0, T ; \mathbf{H}_{0}^{1}(\Omega)\right)$ imply that $\overline{\mathbf{y}} \in L^{2}(0, T ; \mathbf{Y})$. Now, let us take $\boldsymbol{\psi} \in \mathbf{H}^{2}(\Omega) \cap \mathbf{Y}$ and $\phi \in C^{1}[0, T]$ arbitrary. Let $\boldsymbol{\psi}_{h} \in \mathbf{Y}_{h}$ such that $\boldsymbol{\psi}_{h} \rightarrow \boldsymbol{\psi}$ strongly in $\mathbf{W}_{0}^{1,4}(\Omega)$; see (4.5). Then from (4.9) we obtain

$$
\begin{gathered}
\int_{0}^{T}\left(\overline{\mathbf{y}}_{\sigma}(t), \boldsymbol{\psi}_{h}\right) \phi^{\prime}(t) d t=\sum_{n=1}^{N_{\tau}} \int_{t_{n-1}}^{t_{n}}\left(\overline{\mathbf{y}}_{n, h}, \boldsymbol{\psi}_{h}\right) \phi^{\prime}(t) d t=\sum_{n=1}^{N_{\tau}}\left(\overline{\mathbf{y}}_{n, h}, \boldsymbol{\psi}_{h}\right)\left(\phi\left(t_{n}\right)-\phi\left(t_{n-1}\right)\right) \\
=-\sum_{n=1}^{N_{\tau}}\left(\overline{\mathbf{y}}_{n, h}-\overline{\mathbf{y}}_{n-1, h}, \boldsymbol{\psi}_{h}\right) \phi\left(t_{n-1}\right)+\left(\overline{\mathbf{y}}_{N_{\tau}, h}, \boldsymbol{\psi}_{h}\right) \phi(T)-\left(\mathbf{y}_{0 h}, \boldsymbol{\psi}_{h}\right) \phi(0) \\
=\sum_{n=1}^{N_{\tau}}\left\{\tau_{n} a\left(\overline{\mathbf{y}}_{n, h}, \boldsymbol{\psi}_{h}\right)+\tau_{n} c\left(\overline{\mathbf{y}}_{n, h}, \overline{\mathbf{y}}_{n, h}, \boldsymbol{\psi}_{h}\right)-\tau_{n}\left(\mathbf{f}_{n}+\overline{\mathbf{u}}_{n}, \boldsymbol{\psi}_{h}\right)\right\} \phi\left(t_{n-1}\right) \\
+\left(\mathbf{y}_{\sigma}(T), \boldsymbol{\psi}_{h}\right) \phi(T)-\left(\mathbf{y}_{0 h}, \boldsymbol{\psi}_{h}\right) \phi(0) \\
=\int_{0}^{T}\left\{a\left(\overline{\mathbf{y}}_{\sigma}(t), \boldsymbol{\psi}_{h}\right)+c\left(\overline{\mathbf{y}}_{\sigma}(t), \overline{\mathbf{y}}_{\sigma}(t), \boldsymbol{\psi}_{h}\right)-\left(\mathbf{f}(t)+\overline{\mathbf{u}}_{\sigma}(t), \boldsymbol{\psi}_{h}\right)\right\} \phi(t) d t \\
+\sum_{n=1}^{N_{\tau}} \int_{t_{n-1}}^{t_{n}}\left\{a\left(\overline{\mathbf{y}}_{\sigma}(t), \boldsymbol{\psi}_{h}\right)+c\left(\overline{\mathbf{y}}_{\sigma}(t), \overline{\mathbf{y}}_{\sigma}(t), \boldsymbol{\psi}_{h}\right)-\left(\mathbf{f}(t)+\overline{\mathbf{u}}_{\sigma}(t), \boldsymbol{\psi}_{h}\right)\right\}\left(\phi\left(t_{n-1}\right)-\phi(t)\right) d t \\
+\left(\overline{\mathbf{y}}_{\sigma}(T), \boldsymbol{\psi}_{h}\right) \phi(T)-\left(\mathbf{y}_{0 h}, \boldsymbol{\psi}_{h}\right) \phi(0)=I_{1}+I_{2}+I_{3}-I_{4} .
\end{gathered}
$$

Let us study the convergence of these terms when $\sigma \rightarrow 0$. Let us start with $I_{3}$ and $I_{4}$. From the weak convergence $\overline{\mathbf{y}}_{\sigma}(T) \rightarrow \overline{\mathbf{y}}_{T}$ in $\mathbf{L}^{2}(\Omega)$, (4.11), and the strong convergence $\psi_{h} \rightarrow \boldsymbol{\psi}$ in $\mathbf{W}_{0}^{1,4}(\Omega)$, we get

$$
\lim _{\sigma \rightarrow 0}\left(\mathbf{y}_{\sigma}(T), \boldsymbol{\psi}_{h}\right) \phi(T)=\left(\overline{\mathbf{y}}_{T}, \boldsymbol{\psi}\right) \phi(T) \text { and } \lim _{\sigma \rightarrow 0}\left(\mathbf{y}_{0 h}, \boldsymbol{\psi}_{h}\right) \phi(0)=\left(\mathbf{y}_{0}, \boldsymbol{\psi}\right) \phi(0) .
$$

Now we consider the term $I_{1}$. We will prove that

$$
\begin{aligned}
\lim _{\sigma \rightarrow 0} \int_{0}^{T}\left\{a\left(\overline{\mathbf{y}}_{\sigma}(t), \boldsymbol{\psi}_{h}\right)+c\left(\overline{\mathbf{y}}_{\sigma}(t), \overline{\mathbf{y}}_{\sigma}(t), \boldsymbol{\psi}_{h}\right)-\left(\mathbf{f}(t)+\overline{\mathbf{u}}_{\sigma}(t), \boldsymbol{\psi}_{h}\right)\right\} \phi(t) d t \\
=\int_{0}^{T}\{a(\overline{\mathbf{y}}(t), \boldsymbol{\psi})+c(\overline{\mathbf{y}}(t), \overline{\mathbf{y}}(t), \boldsymbol{\psi})-(\mathbf{f}(t)+\overline{\mathbf{u}}(t), \boldsymbol{\psi})\} \phi(t) d t .
\end{aligned}
$$

Copyright $@$ by SIAM. Unauthorized reproduction of this article is prohibited. 
Using again the strong convergence $\boldsymbol{\psi}_{h} \rightarrow \boldsymbol{\psi}$ in $\mathbf{W}_{0}^{1,4}(\Omega)$ and the weak convergences $\overline{\mathbf{u}}_{\sigma} \rightarrow \overline{\mathbf{u}}$ in $L^{2}\left(0, T ; \mathbf{L}^{2}(\Omega)\right)$ and $\overline{\mathbf{y}}_{\sigma} \rightarrow \overline{\mathbf{y}}$ in $L^{2}\left(0, T ; \mathbf{H}_{0}^{1}(\Omega)\right)$, it is immediate to pass to the limit in the first and third terms of the integral. Let us analyze the second term. We have

$$
=c\left(\overline{\mathbf{y}}_{\sigma}(t)-\overline{\mathbf{y}}(t), \overline{\mathbf{y}}_{\sigma}(t), \boldsymbol{\psi}_{h}\right)+c\left(\overline{\mathbf{y}}(t), \overline{\mathbf{y}}_{\sigma}(t)-\overline{\mathbf{y}}(t), \boldsymbol{\psi}_{h}\right)+c\left(\overline{\mathbf{y}}(t), \overline{\mathbf{y}}(t), \boldsymbol{\psi}_{h}-\boldsymbol{\psi}\right) .
$$

For the first term we proceed as follows:

$$
\begin{gathered}
\left|c\left(\overline{\mathbf{y}}_{\sigma}(t)-\overline{\mathbf{y}}(t), \overline{\mathbf{y}}_{\sigma}(t), \boldsymbol{\psi}_{h}\right)\right|=\left|c\left(\overline{\mathbf{y}}_{\sigma}(t)-\overline{\mathbf{y}}(t), \boldsymbol{\psi}_{h}, \overline{\mathbf{y}}_{\sigma}(t)\right)\right| \\
\leq\left\|\overline{\mathbf{y}}_{\sigma}(t)-\overline{\mathbf{y}}(t)\right\|_{\mathbf{L}^{2}(\Omega)}\left\|\nabla \boldsymbol{\psi}_{h}\right\|_{\mathbf{L}^{4}(\Omega)}\left\|\overline{\mathbf{y}}_{\sigma}(t)\right\|_{\mathbf{L}^{4}(\Omega)} .
\end{gathered}
$$

From the boundedness of $\left\{\overline{\mathbf{y}}_{\sigma}\right\}_{\sigma}$ in $L^{8}\left(0, T ; \mathbf{L}^{4}(\Omega)\right)$ and the strong convergence $\overline{\mathbf{y}}_{\sigma} \rightarrow \overline{\mathbf{y}}$ in $L^{2}\left(0, T ; \mathbf{L}^{2}(\Omega)\right)$, we deduce from the above inequality

$$
\begin{gathered}
\int_{0}^{T}\left|c\left(\overline{\mathbf{y}}_{\sigma}(t)-\overline{\mathbf{y}}(t), \overline{\mathbf{y}}_{\sigma}(t), \boldsymbol{\psi}_{h}\right) \| \phi(t)\right| d t \\
\leq\left\|\overline{\mathbf{y}}_{\sigma}-\overline{\mathbf{y}}\right\|_{L^{2}\left(0, T ; \mathbf{L}^{2}(\Omega)\right)}\left\|\nabla \boldsymbol{\psi}_{h}\right\|_{\mathbf{L}^{4}(\Omega)}\left\|\overline{\mathbf{y}}_{\sigma}\right\|_{L^{2}\left(0, T ; \mathbf{L}^{4}(\Omega)\right)}\|\phi\|_{L^{\infty}(0, T)} \rightarrow 0 \text { as } \sigma \rightarrow 0 .
\end{gathered}
$$

In the same way, we obtain

$$
\lim _{\sigma \rightarrow 0} \int_{0}^{T}\left|c\left(\overline{\mathbf{y}}_{\sigma}(t), \overline{\mathbf{y}}_{\sigma}(t)-\overline{\mathbf{y}}(t), \boldsymbol{\psi}_{h}\right)\right||\phi(t)| d t=0 .
$$

For the third term of (4.19), we have

$$
\begin{gathered}
\lim _{\sigma \rightarrow 0} \int_{0}^{T}\left|c\left(\overline{\mathbf{y}}(t), \overline{\mathbf{y}}(t), \boldsymbol{\psi}_{h}-\boldsymbol{\psi}\right)\left\|\phi(t)\left|d t=\lim _{\sigma \rightarrow 0} \int_{0}^{T}\right| c\left(\overline{\mathbf{y}}(t), \boldsymbol{\psi}_{h}-\boldsymbol{\psi}, \overline{\mathbf{y}}(t)\right)\right\| \phi(t)\right| d t \\
\leq\|\overline{\mathbf{y}}\|_{L^{2}\left(0, T ; \mathbf{L}^{4}(\Omega)\right)}^{2}\|\phi\|_{L^{\infty}(0, T)} \lim _{h \rightarrow 0}\left\|\boldsymbol{\psi}_{h}-\boldsymbol{\psi}\right\|_{\mathbf{W}^{1,4}(\Omega)}=0
\end{gathered}
$$

which concludes the proof of (4.18). Finally, we consider the term $I_{2}$. To this end, we observe that

$$
\left|\phi\left(t_{n-1}\right)-\phi(t)\right| \leq\left\|\phi^{\prime}\right\|_{L^{\infty}(0, T)} \tau_{n} \leq\left\|\phi^{\prime}\right\|_{L^{\infty}(0, T)} \tau \quad \forall t \in\left[t_{n-1}, t_{n}\right] \text { and } 1 \leq n \leq N_{\tau} .
$$

Hence, using the boundedness of $\left\{\overline{\mathbf{y}}_{\sigma}\right\}_{\sigma}$ in $L^{8}\left(0, T ; \mathbf{L}^{4}(\Omega)\right) \cap L^{2}\left(0, T ; \mathbf{H}_{0}^{1}(\Omega)\right)$, we get that $I_{2} \rightarrow 0$ when $\sigma \rightarrow 0$. Using the convergence $\overline{\mathbf{y}}_{\sigma} \rightarrow \overline{\mathbf{y}}$ in $L^{2}\left(0, T ; \mathbf{L}^{2}(\Omega)\right)$ and $\boldsymbol{\psi}_{h} \rightarrow \boldsymbol{\psi}$ in $\mathbf{L}^{2}(\Omega)$, we get the desired identity,

$$
\begin{gathered}
\int_{0}^{T}(\mathbf{y}(t), \boldsymbol{\psi}) \phi^{\prime}(t) d t=\lim _{\sigma \rightarrow 0} \int_{0}^{T}\left(\overline{\mathbf{y}}_{\sigma}, \boldsymbol{\psi}_{h}\right) \phi^{\prime}(t) d t \\
=\int_{0}^{T}\{a(\overline{\mathbf{y}}(t), \boldsymbol{\psi})+c(\overline{\mathbf{y}}(t), \overline{\mathbf{y}}(t), \boldsymbol{\psi})-(\mathbf{f}(t)+\overline{\mathbf{u}}(t), \boldsymbol{\psi})\} \phi(t) d t \\
+\left(\overline{\mathbf{y}}_{T}, \boldsymbol{\psi}\right) \phi(T)-\left(\mathbf{y}_{0}, \boldsymbol{\psi}\right) \phi(0) \quad \forall \phi \in C^{1}[0, T] \text { and } \forall \boldsymbol{\psi} \in \mathbf{H}^{2}(\Omega) \cap \mathbf{Y} .
\end{gathered}
$$

By the density of $\mathbf{H}^{2}(\Omega) \cap \mathbf{Y}$ in $\mathbf{Y}$ and the fact that $\overline{\mathbf{y}} \in L^{2}(0, T ; \mathbf{Y})$, the above identity is also valid $\forall \boldsymbol{\psi} \in \mathbf{Y}$. If we take $\phi \in C_{0}^{\infty}(0, T)$, we obtain from (4.20) that the first equation of (2.1) holds in the distributional sense with $\mathbf{f}+\overline{\mathbf{u}}$ in the right-hand side. Therefore, $\overline{\mathbf{y}}_{t} \in L^{2}\left(0, T ; \mathbf{Y}^{*}\right)$, and hence $\overline{\mathbf{y}} \in \mathbf{W}(0, T)$ holds. Moreover, the functions

Copyright (c) by SIAM. Unauthorized reproduction of this article is prohibited. 
$t \in(0, T) \mapsto g(t)=(\overline{\mathbf{y}}(t), \boldsymbol{\psi}) \in \mathbb{R}$ and $t \in(0, T) \mapsto g^{\prime}(t)=\left(\overline{\mathbf{y}}_{t}(t), \boldsymbol{\psi}\right) \in \mathbb{R}$ belong to $L^{2}(0, T)$. Therefore, $g$ belongs to $H^{1}(0, T) \subset C[0, T]$, and the following integration by parts is valid:

$$
\begin{aligned}
(\overline{\mathbf{y}}(T), \boldsymbol{\psi}) \phi(T) & -(\overline{\mathbf{y}}(0), \boldsymbol{\psi}) \phi(0)=\int_{0}^{T}\left(\overline{\mathbf{y}}_{t}(t), \boldsymbol{\psi}\right) \phi(t) d t+\int_{0}^{T}(\overline{\mathbf{y}}(t), \boldsymbol{\psi}) \phi^{\prime}(t) d t \\
& =\left(\overline{\mathbf{y}}_{T}, \boldsymbol{\psi}\right) \phi(T)-\left(\mathbf{y}_{0}, \boldsymbol{\psi}\right) \phi(0) \quad \forall \phi \in C^{1}[0, T] .
\end{aligned}
$$

Selecting $\phi \in C^{1}[0, T]$ with $\phi(T)=0$ and $\phi(0)=1$ and taking into account that $\boldsymbol{\psi}$ is an arbitrary element of $\mathbf{Y}$, we deduce that $\overline{\mathbf{y}}(0)=\mathbf{y}_{0}$. Thus, we get that $(\overline{\mathbf{u}}, \overline{\mathbf{y}})$ satisfies (2.1). Analogously, we can take $\phi$ satisfying $\phi(0)=0$ and $\phi(T)=1$ to deduce that $\overline{\mathbf{y}}(T)=\overline{\mathbf{y}}_{T}$ as desired.

Now we prove a converse result. The next theorem states that any strict local minimum of $(\mathrm{P})$ can be approximated by a sequence of local minima of problems $\left(\mathrm{P}_{\sigma}\right)$.

THEOREM 4.3. Let us assume that (4.12) holds, and let $\overline{\mathbf{u}}$ be a strict local minimum of $(\mathrm{P})$ in the $L^{2}\left(0, T ; \mathbf{L}^{2}(\Omega)\right)$ sense. Then there exist a number $\bar{\mu}_{0}>0$ and a sequence $\left\{\left(\overline{\mathbf{u}}_{\sigma}, \overline{\mathbf{y}}_{\sigma}\right)\right\}_{|\sigma| \leq \bar{\mu}_{0}}$ of local minima of problems $\left(\mathrm{P}_{\sigma}\right)$ such that (4.15) and (4.16) hold with $\overline{\mathbf{y}}=G(\overline{\mathbf{u}})$. Moreover, $\left\{\overline{\mathbf{u}}_{\sigma}\right\}_{|\sigma| \leq \bar{\mu}_{0}} \subset \mathcal{A}$, (4.9) has a unique solution for every $\overline{\mathbf{u}}_{\sigma}$ with $|\sigma| \leq \bar{\mu}_{0}$, and there exists $\varepsilon>0$ such that $\mathcal{J}_{\sigma}$ attains its minimum value in $\left(\bar{B}_{\varepsilon}(\overline{\mathbf{u}}) \cap \mathcal{U}_{\sigma, a d}\right) \times \mathcal{Y}_{\sigma}$ at $\left(\overline{\mathbf{u}}_{\sigma}, \overline{\mathbf{y}}_{\sigma}\right)$ for every $|\sigma| \leq \bar{\mu}_{0}$.

Proof. Since $\overline{\mathbf{u}}$ is a strict local minimum of $(\mathrm{P})$, there exists $\varepsilon>0$ such that $\overline{\mathbf{u}}$ is the unique solution of the control problem

$$
\text { (Q) }\left\{\begin{array}{l}
\min J(\mathbf{u}) \\
\mathbf{u} \in \bar{B}_{\varepsilon}(\overline{\mathbf{u}}) \cap \mathcal{U}_{a d},
\end{array}\right.
$$

where $\varepsilon$ is taken sufficiently small so that $\bar{B}_{\varepsilon}(\overline{\mathbf{u}}) \subset \mathcal{A}$. Along this proof, every element $\mathbf{u}_{\sigma} \in \mathcal{U}_{\sigma}$ will be extended to $\Omega \times(0, T)$ by setting $\mathbf{u}_{\sigma}(t, x)=\overline{\mathbf{u}}(t, x)$ for almost every $(t, x) \in(0, T) \times\left(\Omega \backslash \Omega_{h}\right)$.

Now we consider the discrete control problem

$$
\left(\mathrm{Q}_{\sigma}\right) \min \left\{\mathcal{J}_{\sigma}\left(\mathbf{u}_{\sigma}, \mathbf{y}_{\sigma}\right):\left(\mathbf{u}_{\sigma}, \mathbf{y}_{\sigma}\right) \in\left(\bar{B}_{\varepsilon}(\overline{\mathbf{u}}) \cap \mathcal{U}_{\sigma, a d}\right) \times \mathcal{Y}_{\sigma} \text { satisfy }(4.9)\right\} .
$$

From the continuity and coercivity of $\mathcal{J}_{\sigma}$ and the fact that the set of admissible points is closed, it is enough to prove that $\left(\mathrm{Q}_{\sigma}\right)$ has at least one admissible point to deduce the existence of a solution. To this end, we consider the $L^{2}\left(0, T ; \mathbf{L}^{2}(\Omega)\right)$-projection $\mathbf{u}_{\sigma}$ of $\overline{\mathbf{u}}$. It is obvious that $\mathbf{u}_{\sigma} \in \mathcal{U}_{\sigma, a d}$ for every $\sigma$ and $\left\|\overline{\mathbf{u}}-\mathbf{u}_{\sigma}\right\|_{L^{2}\left(0, T ; \mathbf{L}^{2}(\Omega)\right)} \rightarrow 0$ as $\sigma \rightarrow 0$; then there exists $\mu_{1}>0$ such that $\mathbf{u}_{\sigma} \in \bar{B}_{\varepsilon}(\overline{\mathbf{u}})$ for every $|\sigma| \leq \mu_{1}$, which shows that $\mathbf{u}_{\sigma}$ is an admissible point of $\left(\mathrm{Q}_{\sigma}\right)$ for every $|\sigma| \leq \mu_{1}$. Let $\left(\overline{\mathbf{u}}_{\sigma}, \overline{\mathbf{y}}_{\sigma}\right)$ be a solution of $\left(\mathrm{Q}_{\sigma}\right)$. Then, arguing as in the proof of Theorem 4.2, we obtain that (4.15) and (4.16) hold. Furthermore, applying Theorem 4.1, we deduce the existence of $0<\bar{\mu}_{0} \leq \mu_{1}$ such that (4.9) has a unique solution for every $\overline{\mathbf{u}}_{\sigma}$ with $|\sigma| \leq \bar{\mu}_{0}$. Using (4.15) and taking $\bar{\mu}_{0}$ sufficiently small, we have that $\overline{\mathbf{u}}_{\sigma} \in B_{\varepsilon}(\overline{\mathbf{u}})$. Hence, $\left(\overline{\mathbf{u}}_{\sigma}, \overline{\mathbf{y}}_{\sigma}\right)$ is a local minimum of $\left(\mathrm{P}_{\sigma}\right)$, and $\mathcal{J}_{\sigma}$ attains the minimum value in $\left(\bar{B}_{\varepsilon}(\overline{\mathbf{u}}) \cap \mathcal{U}_{\sigma, a d}\right) \times \mathcal{Y}_{\sigma}$ at this point.

In the rest of this section, $\overline{\mathbf{u}}$ will denote a local (or global) minimum of $(\mathrm{P})$ with associated state and adjoint state $\overline{\mathbf{y}}$ and $\overline{\boldsymbol{\varphi}}$, respectively. In addition, $\left\{\left(\overline{\mathbf{u}}_{\sigma}, \overline{\mathbf{y}}_{\sigma}\right)\right\}_{|\sigma| \leq \bar{\mu}_{0}}$ will be a sequence of local (or global) minima of problems $\left(\mathrm{P}_{\sigma}\right)$ satisfying (4.15) and (4.16). The goal is to get the rate of convergence of $\left(\overline{\mathbf{u}}-\overline{\mathbf{u}}_{\sigma}, \overline{\mathbf{y}}-\overline{\mathbf{y}}_{\sigma}, \overline{\boldsymbol{\varphi}}-\overline{\boldsymbol{\varphi}}_{\sigma}\right)$, where $\overline{\boldsymbol{\varphi}}_{\sigma}$ denotes the discrete adjoint state associated to $\overline{\mathbf{u}}_{\sigma}$. To this end we assume that (3.19) and (4.12) hold. The first step in the derivation of the error estimates is to prove 
that $\overline{\boldsymbol{\varphi}}_{\sigma}$ is well defined and to write the first order optimality conditions satisfied by $\left(\overline{\mathbf{u}}_{\sigma}, \overline{\mathbf{y}}_{\sigma}\right)$. Since $\overline{\mathbf{u}} \in \mathcal{U}_{a d} \subset \mathcal{A}$, we know that $\overline{\mathbf{y}} \in \mathbf{H}^{2,1}\left(\Omega_{T}\right)$. Let $B(\overline{\mathbf{y}})$ denote the unit ball centered at $\overline{\mathbf{y}}$ in $\mathbf{H}^{2,1}\left(\Omega_{T}\right)$. Since $G: \mathcal{A} \longrightarrow \mathbf{H}^{2,1}\left(\Omega_{T}\right) \cap C([0, T], \mathbf{Y})$ is continuous and $G(\overline{\mathbf{u}})=\overline{\mathbf{y}}$, there exists $\varepsilon>0$ such that $B_{\varepsilon}(\overline{\mathbf{u}}) \subset \mathcal{A}$ and $G\left(B_{\varepsilon}(\overline{\mathbf{u}})\right) \subset B(\overline{\mathbf{y}})$. From the embedding $\mathbf{H}^{2,1}\left(\Omega_{T}\right) \subset L^{8}\left(0, T ; \mathbf{L}^{4}(\Omega)\right)$ we have that $\mathbf{K}=B_{\varepsilon}(\overline{\mathbf{u}}) \times B(\overline{\mathbf{y}})$ is a bounded subset of $L^{2}\left(0, T ; \mathbf{L}^{2}(\Omega)\right) \times L^{8}\left(0, T ; \mathbf{L}^{4}(\Omega)\right)$. Moreover, $\left(\mathbf{u}, \mathbf{y}_{\mathbf{u}}\right) \in \mathbf{K} \forall \mathbf{u} \in$ $B_{\varepsilon}(\overline{\mathbf{u}})$. Hence, Theorem 4.1 implies the existence of $\tau_{\mathbf{K}}>0$ such that (4.9) has a unique solution for every $\mathbf{u} \in B_{\varepsilon}(\overline{\mathbf{u}})$ and all $\tau<\tau_{\mathbf{K}}$. Now, using (4.15) we deduce the existence of $\sigma_{0} \in\left(0, \tau_{\mathbf{K}}\right)$ such that $\overline{\mathbf{u}}_{\sigma} \in B_{\varepsilon}(\overline{\mathbf{u}})$ and that $\overline{\mathbf{y}}_{\sigma}$ is the unique solution of (4.9) associated to $\overline{\mathbf{u}}_{\sigma}$ for every $|\sigma| \leq \sigma_{0}$. Hence, for $|\sigma| \leq \sigma_{0}$ we can define the functions

$$
J_{\sigma}: B_{\varepsilon}(\overline{\mathbf{u}}) \rightarrow \mathbb{R}, \quad J_{\sigma}(\mathbf{u})=\mathcal{J}_{\sigma}\left(\mathbf{u}, \mathbf{y}_{\sigma}(\mathbf{u})\right) .
$$

Moreover, $\overline{\mathbf{u}}_{\sigma}$ is a local (global) minimum of $J_{\sigma}$ in $B_{\varepsilon}(\overline{\mathbf{u}}) \cap \mathcal{U}_{\sigma, a d}$. We need to prove the differentiability of $J_{\sigma}$ to get the optimality conditions satisfied by $\overline{\mathbf{u}}_{\sigma}$. The reader is referred to [5, sect. 4.2] for the proof of the following theorem.

TheOREM 4.4. For every $|\sigma| \leq \sigma_{0}$ the function $J_{\sigma}: B_{\varepsilon}(\overline{\mathbf{u}}) \rightarrow \mathbb{R}$ is of class $C^{\infty}$ and

$$
J_{\sigma}^{\prime}(\mathbf{u}) \mathbf{v}=\int_{0}^{T} \int_{\Omega_{h}}\left(\boldsymbol{\varphi}_{\sigma}+\lambda \mathbf{u}\right) \mathbf{v} d x d t
$$

where $\varphi_{\sigma} \in \mathcal{Y}_{\sigma}$ is the solution of the adjoint equation

$$
\left\{\begin{array}{l}
\text { for } n=N_{\tau}, \ldots, 1, \quad \text { and } \forall \boldsymbol{\psi}_{h} \in \mathbf{Y}_{h}, \\
\left(\frac{\boldsymbol{\varphi}_{n, h}-\boldsymbol{\varphi}_{n+1, h}}{\tau_{n}}, \boldsymbol{\psi}_{h}\right)+a\left(\boldsymbol{\varphi}_{n, h}, \boldsymbol{\psi}_{h}\right)+c\left(\boldsymbol{\psi}_{h}, \mathbf{y}_{n, h}, \boldsymbol{\varphi}_{n, h}\right)+c\left(\mathbf{y}_{n, h}, \boldsymbol{\psi}_{h}, \boldsymbol{\varphi}_{n, h}\right) \\
=\frac{1}{\tau_{n}} \int_{t_{n-1}}^{t_{n}}\left\|\mathbf{y}_{n, h}-\mathbf{y}_{d}\right\|_{\mathbf{L}^{4}\left(\Omega_{h}\right)}^{4}\left(\left|\mathbf{y}_{n, h}-\mathbf{y}_{d}\right|^{2}\left(\mathbf{y}_{n, h}-\mathbf{y}_{d}\right), \boldsymbol{\psi}_{h}\right) \mathrm{d} t \\
\boldsymbol{\varphi}_{N_{\tau}+1, h}=\gamma\left(\mathbf{y}_{N_{\tau}, h}-\mathbf{y}_{\Omega_{h}}\right),
\end{array}\right.
$$

and $\mathbf{y}_{\sigma}$ is the solution of (4.9) corresponding to $\mathbf{u}$.

The optimality conditions for $\overline{\mathbf{u}}_{\sigma}$ can be written as $J_{\sigma}^{\prime}\left(\overline{\mathbf{u}}_{\sigma}\right)\left(\mathbf{u}_{\sigma}-\overline{\mathbf{u}}_{\sigma}\right) \geq 0 \forall \mathbf{u} \in$ $\mathcal{U}_{\sigma, a d}$. Using (4.21) we get the following corollary.

COROLlaRY 4.5. With the above notation, there exist $\overline{\mathbf{y}}_{\sigma}, \overline{\boldsymbol{\varphi}}_{\sigma} \in \mathcal{Y}_{\sigma}$ such that

$$
\begin{aligned}
& \left\{\begin{array}{l}
\text { For } n=1, \ldots, N_{\tau}, \text { and } \forall \boldsymbol{\psi}_{h} \in \mathbf{Y}_{h}, \\
\left(\frac{\overline{\mathbf{y}}_{n, h}-\overline{\mathbf{y}}_{n-1, h}}{\tau_{n}}, \boldsymbol{\psi}_{h}\right)+a\left(\overline{\mathbf{y}}_{n, h}, \boldsymbol{\psi}_{h}\right)+c\left(\overline{\mathbf{y}}_{n, h}, \overline{\mathbf{y}}_{n, h}, \boldsymbol{\psi}_{h}\right)=\left(\mathbf{f}_{n}+\mathbf{u}_{n}, \boldsymbol{\psi}_{h}\right), \\
\overline{\mathbf{y}}_{0, h}=\mathbf{y}_{0 h},
\end{array}\right. \\
& \left\{\begin{array}{l}
\text { for } n=N_{\tau}, \ldots, 1, \quad \text { and } \forall \boldsymbol{\psi}_{h} \in \mathbf{Y}_{h}, \\
\left(\frac{\overline{\boldsymbol{\varphi}}_{n, h}-\overline{\boldsymbol{\varphi}}_{n+1, h}}{\tau_{n}}, \boldsymbol{\psi}_{h}\right)+a\left(\overline{\boldsymbol{\varphi}}_{n, h}, \boldsymbol{\psi}_{h}\right)+c\left(\boldsymbol{\psi}_{h}, \overline{\mathbf{y}}_{n, h}, \overline{\boldsymbol{\varphi}}_{n, h}\right)+c\left(\overline{\mathbf{y}}_{n, h}, \boldsymbol{\psi}_{h}, \overline{\boldsymbol{\varphi}}_{n, h}\right) \\
=\frac{1}{\tau_{n}} \int_{t_{n-1}}^{t_{n}}\left\|\overline{\mathbf{y}}_{n, h}-\mathbf{y}_{d}\right\|_{\mathbf{L}^{4}\left(\Omega_{h}\right)}^{4}\left(\left|\overline{\mathbf{y}}_{n, h}-\mathbf{y}_{d}\right|^{2}\left(\overline{\mathbf{y}}_{n, h}-\mathbf{y}_{d}\right), \boldsymbol{\psi}_{h}\right) \mathrm{d} t, \\
\overline{\boldsymbol{\varphi}}_{N_{\tau}+1, h}=\gamma\left(\mathbf{y}_{N_{\tau}, h}-\mathbf{y}_{\Omega_{h}}\right),
\end{array}\right. \\
& \int_{0}^{T} \int_{\Omega_{h}}\left(\overline{\boldsymbol{\varphi}}_{\sigma}+\lambda \overline{\mathbf{u}}_{\sigma}\right)\left(\mathbf{u}_{\sigma}-\overline{\mathbf{u}}_{\sigma}\right) d x d t \geq 0 \quad \forall \mathbf{u}_{\sigma} \in \mathcal{U}_{\sigma, a d} .
\end{aligned}
$$

Copyright $@$ ㅇ by SIAM. Unauthorized reproduction of this article is prohibited. 
Analogously to Theorem 4.1 we have the following result, whose proof follows from [5, Cor. 4.12].

THEOREM 4.6. Under assumption (4.12), there exists a constant $C>0$ independent of $\sigma$ such that $\forall \mathbf{u}, \mathbf{v} \in B_{\varepsilon}(\overline{\mathbf{u}})$ and $|\sigma| \leq \sigma_{0}$,

$$
\begin{aligned}
& \left\|\boldsymbol{\varphi}_{\mathbf{u}}-\boldsymbol{\varphi}_{\sigma}(\mathbf{v})\right\|_{L^{\infty}\left(0, T ; \mathbf{L}^{2}\left(\Omega_{h}\right)\right)}+\left\|\boldsymbol{\varphi}_{\mathbf{u}}-\boldsymbol{\varphi}_{\sigma}(\mathbf{v})\right\|_{L^{2}\left(0, T ; \mathbf{H}^{1}\left(\Omega_{h}\right)\right)} \\
& \leq C\left\{h+\|\mathbf{u}-\mathbf{v}\|_{L^{2}\left(0, T ; \mathbf{L}^{2}(\Omega)\right)}\right\} .
\end{aligned}
$$

Proof (sketch). Let $\mathbf{y}_{\mathbf{u}}$ be the associated state to $\mathbf{u}$, let $\boldsymbol{\varphi} \equiv \boldsymbol{\varphi}_{\mathbf{u}}$ denote the associated adjoint state, and let $\varphi_{\sigma} \equiv \varphi_{\sigma}(\mathbf{u})$ denote the associated discrete state. First, we prove that $\left\|\boldsymbol{\varphi}-\boldsymbol{\varphi}_{\sigma}\right\|_{L^{\infty}\left(0, T ; \mathbf{L}^{2}\left(\Omega_{h}\right)\right)}+\left\|\boldsymbol{\varphi}-\boldsymbol{\varphi}_{\sigma}\right\|_{L^{2}\left(0, T ; \mathbf{H}^{1}\left(\Omega_{h}\right)\right)} \leq C h$, where $C$ is a constant depending on the $L^{\infty}\left(0, T ; \mathbf{L}^{6}(\Omega)\right)$ and $L^{2}\left(0, T ; \mathbf{L}^{\infty}(\Omega)\right)$ norms of $\mathbf{y}_{d}$, and the $L^{\infty}\left(0, T ; \mathbf{H}^{1}\left(\Omega_{h}\right)\right)$ norms of $\mathbf{y}, \mathbf{y}_{\sigma}, \boldsymbol{\varphi}$. Then the result follows similarly to [5, Cor. 4.12]. We consider the projection operator $\mathcal{R}_{\sigma}: C\left(0, T ; \mathbf{L}^{2}\left(\Omega_{h}\right)\right) \rightarrow \mathcal{Y}_{\sigma}$ by $\left(\mathcal{R}_{\sigma} \mathbf{w}\right)_{n, h}=P_{h} \mathbf{w}\left(t_{n-1}\right)$ for $1 \leq n \leq N_{\tau}$, where $P_{h}$ is the standard $\mathbf{L}^{2}(\Omega)$-projection, i.e., $P_{h}: \mathbf{L}^{2}(\Omega) \rightarrow \mathbf{Y}_{h}$, with $\left(P_{h} \mathbf{y}, \mathbf{w}_{h}\right)=\left(\mathbf{y}, \mathbf{w}_{h}\right), \forall \mathbf{w}_{h} \in \mathbf{Y}_{h}$. In addition, for the discrete adjoint states, we fix $\left(\mathcal{R}_{\sigma} \mathbf{w}\right)\left(t_{n-1}\right)=(\mathcal{R} \mathbf{w})_{n, h}$. We split the error into $\boldsymbol{\epsilon}=\boldsymbol{\varphi}-\boldsymbol{\varphi}_{\sigma}=\left(\boldsymbol{\varphi}-R_{\sigma} \varphi\right)+\left(R_{\sigma} \varphi-\varphi_{\sigma}\right)=\boldsymbol{\eta}+\boldsymbol{\epsilon}_{\sigma}$ and note that according to our notation, we have $\boldsymbol{\eta}\left(t_{n}\right)=\boldsymbol{\varphi}\left(t_{n}\right)-\left(R_{\sigma} \varphi\right)\left(t_{n}\right)=\boldsymbol{\varphi}\left(t_{n}\right)-\left(R_{\sigma} \varphi\right)_{n+1, h}=\boldsymbol{\varphi}\left(t_{n}\right)-$ $P_{h} \varphi\left(t_{n}\right)$ for $0 \leq n \leq N_{\tau}-1$. Also we have $\epsilon_{\sigma}\left(t_{n}\right)=\epsilon_{n+1, h}, 0 \leq n \leq N_{\tau}-1$. Setting $\left(R_{\sigma} \mathbf{w}\right)_{N_{\tau}+1, h}=P_{h} \mathbf{w}(T)$ and recalling that $\boldsymbol{\varphi}_{N_{\tau}+1, h}=\gamma\left(\mathbf{y}_{N_{\tau}, h}-\mathbf{y}_{\Omega_{h}}\right)$, the previous identities are also well defined for $n=N_{\tau}$. Then working identically to [5, Thm. 4.11], the orthogonality condition related to (3.6) and (4.22) leads to the following inequalities, with $n=N_{\tau}, \ldots, 1$ :

$$
\begin{aligned}
& \frac{1}{2}\left\|\boldsymbol{\epsilon}_{n, h}\right\|^{2}-\frac{1}{2}\left\|\boldsymbol{\epsilon}_{n+1, h}\right\|^{2}+\frac{1}{2}\left\|\boldsymbol{\epsilon}_{n, h}-\boldsymbol{\epsilon}_{n+1, h}\right\|^{2}+\nu \int_{t_{n-1}}^{t_{n}}\left\|\nabla \boldsymbol{\epsilon}_{n, h}\right\|^{2} \mathrm{~d} t \\
& \leq \nu \int_{t_{n-1}}^{t_{n}}\|\nabla \boldsymbol{\eta}(t)\|\left\|\nabla \boldsymbol{\epsilon}_{n, h}\right\| \mathrm{d} t+\int_{t_{n-1}}^{t_{n}}\left[c\left(\mathbf{y}_{n, h}, \boldsymbol{\epsilon}_{n, h}, \boldsymbol{\varphi}_{n, h}\right)-c\left(\mathbf{y}(t), \boldsymbol{\epsilon}_{n, h}, \boldsymbol{\varphi}(t)\right)\right] \mathrm{d} t \\
& +\int_{t_{n-1}}^{t_{n}}\left[c\left(\boldsymbol{\epsilon}_{n, h}, \mathbf{y}_{n, h}, \boldsymbol{\varphi}_{n, h}\right)-c\left(\boldsymbol{\epsilon}_{n, h}, \mathbf{y}(t), \boldsymbol{\varphi}(t)\right)\right] \mathrm{d} t \\
& +\int_{t^{n-1}}^{t^{n}}\left(\left\|\mathbf{y}_{\mathbf{u}}-\mathbf{y}_{d}\right\|_{\mathbf{L}^{4}(\Omega)}^{4}\left|\mathbf{y}-\mathbf{y}_{d}\right|^{2}\left(\mathbf{y}-\mathbf{y}_{d}\right), \boldsymbol{\epsilon}_{n, h}\right) \mathrm{d} t \\
& \quad-\int_{t^{n-1}}^{t^{n}}\left\|\mathbf{y}_{n, h}-\mathbf{y}_{d}\right\|_{\mathbf{L}^{4}\left(\Omega_{h}\right)}^{4}\left(\left|\mathbf{y}_{n, h}-\mathbf{y}_{d}\right|^{2}\left(\mathbf{y}_{n, h}-\mathbf{y}_{d}\right), \boldsymbol{\epsilon}_{n, h}\right) \mathrm{d} t
\end{aligned}
$$

The trilinear terms can be treated similar to [5, Thm. 4.11] after noting that $\mathbf{y}_{\sigma} \in$ $L^{\infty}\left(0, T ; \mathbf{H}^{1}\left(\Omega_{h}\right)\right)$ and $\varphi \in L^{\infty}\left(0, T ; \mathbf{H}^{1}(\Omega)\right)$. Indeed, we can bound the trilinear terms by

$$
\begin{aligned}
& \int_{t_{n-1}}^{t_{n}} \text { trilinear terms } \mid \mathrm{d} t \leq \frac{\nu}{8} \int_{t_{n-1}}^{t_{n}}\left\|\boldsymbol{\epsilon}_{n, h}\right\|_{\mathbf{L}^{2}\left(\Omega_{h}\right)}^{2} \mathrm{~d} t+C\left(\int_{t_{n-1}}^{t_{n}}\|\boldsymbol{\eta}\|_{\mathbf{H}^{1}\left(\Omega_{h}\right)}^{2} \mathrm{~d} t\right. \\
&\left.\quad+\int_{t_{n-1}}^{t_{n}}\left\|\mathbf{y}-\mathbf{y}_{n, h}\right\|_{\mathbf{H}^{1}\left(\Omega_{h}\right)}^{2} \mathrm{~d} t\right),
\end{aligned}
$$

where constant $C$ depends on the $L^{\infty}\left(0, T ; \mathbf{H}^{1}(\Omega)\right)$ norms of $\boldsymbol{\varphi}, \mathbf{y}$, and $\mathbf{y}_{\sigma}$. To complete the proof, it remains to estimate the difference of the last two integrals in a 
similar way. Then, the discrete Grönwall inequality finishes the proof. First, note that

$$
\begin{aligned}
I \equiv & \int_{t_{n-1}}^{t_{n}}\left(\left\|\mathbf{y}_{\mathbf{u}}-\mathbf{y}_{d}\right\|_{\mathbf{L}^{4}(\Omega)}^{4}\left|\mathbf{y}-\mathbf{y}_{d}\right|^{2}\left(\mathbf{y}-\mathbf{y}_{d}\right), \boldsymbol{\epsilon}_{n, h}\right) \mathrm{d} t \\
& \quad-\int_{t_{n-1}}^{t_{n}}\left\|\mathbf{y}_{n, h}-\mathbf{y}_{d}\right\|_{\mathbf{L}^{4}\left(\Omega_{h}\right)}^{4}\left(\left|\mathbf{y}_{n, h}-\mathbf{y}_{d}\right|^{2}\left(\mathbf{y}_{n, h}-\mathbf{y}_{d}\right), \boldsymbol{\epsilon}_{n, h}\right) \mathrm{d} t \\
= & \int_{t_{n-1}}^{t_{n}}\left(\left\|\mathbf{y}_{\mathbf{u}}-\mathbf{y}_{d}\right\|_{\mathbf{L}^{4}(\Omega)}^{4}-\left\|\mathbf{y}_{n, h}-\mathbf{y}_{d}\right\|_{\mathbf{L}^{4}\left(\Omega_{h}\right)}^{4}\right)\left(\left|\mathbf{y}-\mathbf{y}_{d}\right|^{2}\left(\mathbf{y}-\mathbf{y}_{d}\right), \boldsymbol{\epsilon}_{n, h}\right) \mathrm{d} t \\
& +\int_{t_{n-1}}^{t_{n}}\left\|\mathbf{y}_{n, h}-\mathbf{y}_{d}\right\|_{\mathbf{L}^{4}\left(\Omega_{h}\right)}^{4}\left(\left|\mathbf{y}-\mathbf{y}_{d}\right|^{2}\left(\mathbf{y}-\mathbf{y}_{d}\right)-\left|\mathbf{y}_{n, h}-\mathbf{y}_{d}\right|^{2}\left(\mathbf{y}_{n, h}-\mathbf{y}_{d}\right), \boldsymbol{\epsilon}_{n, h}\right) \mathrm{d} t \\
= & \int_{t_{n-1}}^{t_{n}}\left\|\mathbf{y}_{\mathbf{u}}-\mathbf{y}_{d}\right\|_{\mathbf{L}^{4}\left(\Omega \backslash \Omega_{h}\right)}^{4}\left(\left|\mathbf{y}-\mathbf{y}_{d}\right|^{2}\left(\mathbf{y}-\mathbf{y}_{d}\right), \boldsymbol{\epsilon}_{n, h}\right) \mathrm{d} t \\
& +\int_{t_{n-1}}^{t_{n}}\left(\left\|\mathbf{y}_{\mathbf{u}}-\mathbf{y}_{d}\right\|_{\mathbf{L}^{4}\left(\Omega_{h}\right)}^{4}-\left\|\mathbf{y}_{n, h}-\mathbf{y}_{d}\right\|_{\mathbf{L}^{4}\left(\Omega_{h}\right)}^{4}\right)\left(\left|\mathbf{y}-\mathbf{y}_{d}\right|^{2}\left(\mathbf{y}-\mathbf{y}_{d}\right), \boldsymbol{\epsilon}_{n, h}\right) \mathrm{d} t \\
& +\int_{t_{n-1}}^{t_{n}}\left\|\mathbf{y}_{n, h}-\mathbf{y}_{d}\right\|_{\mathbf{L}^{4}\left(\Omega_{h}\right)}^{4}\left(\left|\mathbf{y}-\mathbf{y}_{d}\right|^{2}\left(\mathbf{y}-\mathbf{y}_{d}\right)-\left|\mathbf{y}_{n, h}-\mathbf{y}_{d}\right|^{2}\left(\mathbf{y}_{n, h}-\mathbf{y}_{d}\right), \boldsymbol{\epsilon}_{n, h}\right) \mathrm{d} t \\
\equiv & I_{1}+I_{2}+I_{3} .
\end{aligned}
$$

For the first integral, we note that Hölder's inequality, interpolation inequality $\|\cdot\|_{L^{4}} \leq$ $C\|.\|_{L^{2}}^{1 / 4}\|\cdot\|_{L^{6}}^{3 / 4}$, assumption (4.1), and Young's inequality imply

$$
\begin{aligned}
& I_{1} \leq \int_{t_{n-1}}^{t^{n}}\left\|\mathbf{y}-\mathbf{y}_{d}\right\|_{L^{4}\left(\Omega \backslash \Omega_{h}\right)}^{4}\left\|\mathbf{y}-\mathbf{y}_{d}\right\|_{\mathbf{L}^{6}\left(\Omega_{h}\right)}^{3}\left\|\boldsymbol{\epsilon}_{n, h}\right\|_{\mathbf{L}^{2}\left(\Omega_{h}\right)} \mathrm{d} t \\
& \leq C\left\|\mathbf{y}-\mathbf{y}_{d}\right\|_{L^{\infty}\left(0, T ; \mathbf{L}^{6}(\Omega)\right)}^{6} \int_{t_{n-1}}^{t_{n}}\left\|\mathbf{y}-\mathbf{y}_{d}\right\|_{L^{2}\left(\Omega \backslash \Omega_{h}\right)}\left\|\boldsymbol{\epsilon}_{n, h}\right\|_{\mathbf{L}^{2}\left(\Omega_{h}\right)} \mathrm{d} t \\
& \leq C_{\mathbf{y}, \mathbf{y}_{d}} \int_{t_{n-1}}^{t_{n}}\left\|\mathbf{y}-\mathbf{y}_{d}\right\|_{L^{\infty}(\Omega)} h\left\|\boldsymbol{\epsilon}_{n, h}\right\|_{\mathbf{L}^{2}\left(\Omega_{h}\right)} \mathrm{d} t \\
& \leq \int_{t_{n-1}}^{t_{n}}\left\|\boldsymbol{\epsilon}_{n, h}\right\|_{\mathbf{L}^{2}\left(\Omega_{h}\right)}^{2} d t+C h^{2} \int_{t_{n-1}}^{t_{n}}\left\|\mathbf{y}-\mathbf{y}_{d}\right\|_{L^{\infty}(\Omega)}^{2} \mathrm{~d} t
\end{aligned}
$$

where constant $C$ depends on $\left\|\mathbf{y}-\mathbf{y}_{d}\right\|_{L^{\infty}\left(0, T ; \mathbf{L}^{6}(\Omega)\right)}$. For the second integral, using standard algebra, Hölder's inequality, and the stability bounds on $\mathbf{y}, \mathbf{y}_{d}, \mathbf{y}_{\sigma}$ in $L^{\infty}\left(0, T ; \mathbf{L}^{\mathbf{6}}(\Omega)\right)$, we obtain that

$$
\begin{aligned}
& I_{2} \leq \int_{t_{n-1}}^{t_{n}}\left\|\mathbf{y}_{\mathbf{u}}-\mathbf{y}_{n, h}\right\|_{\mathbf{L}^{4}\left(\Omega_{h}\right)} \times\left(\left\|\mathbf{y}_{\mathbf{u}}-\mathbf{y}_{d}\right\|_{\mathbf{L}^{4}\left(\Omega_{h}\right)}+\left\|\mathbf{y}_{n, h}-\mathbf{y}_{d}\right\|_{\mathbf{L}^{4}\left(\Omega_{h}\right)}\right) \\
& \quad \times\left(\left\|\mathbf{y}_{\mathbf{u}}-\mathbf{y}_{d}\right\|_{\mathbf{L}^{4}\left(\Omega_{h}\right)}^{2}+\left\|\mathbf{y}_{n, h}-\mathbf{y}_{d}\right\|_{\mathbf{L}^{4}\left(\Omega_{h}\right)}^{2}\right)\left\|\mathbf{y}-\mathbf{y}_{d}\right\|_{\mathbf{L}^{6}\left(\Omega_{h}\right)}^{3}\left\|\boldsymbol{\epsilon}_{n, h}\right\|_{\mathbf{L}^{2}\left(\Omega_{h}\right)} \mathrm{d} t \\
& \leq C \int_{t_{n-1}}^{t_{n}}\left\|\mathbf{y}_{\mathbf{u}}-\mathbf{y}_{n, h}\right\|_{\mathbf{L}^{4}\left(\Omega_{h}\right)}\left\|\boldsymbol{\epsilon}_{n, h}\right\|_{\mathbf{L}^{2}\left(\Omega_{h}\right)} \mathrm{d} t \\
& \leq \int_{t_{n-1}}^{t_{n}}\left\|\boldsymbol{\epsilon}_{n, h}\right\|_{\mathbf{L}^{2}\left(\Omega_{h}\right)}^{2}+C \int_{t_{n-1}}^{t_{n}}\left\|\mathbf{y}_{\mathbf{u}}-\mathbf{y}_{n, h}\right\|_{\mathbf{H}^{1}\left(\Omega_{h}\right)}^{2} \mathrm{~d} t
\end{aligned}
$$

Copyright $@$ by SIAM. Unauthorized reproduction of this article is prohibited. 
For the third integral, using standard algebra, we arrive at

$$
\begin{aligned}
I_{3}= & \int_{t_{n-1}}^{t_{n}}\left\|\mathbf{y}_{n, h}-\mathbf{y}_{d}\right\|_{\mathbf{L}^{4}\left(\Omega_{h}\right)}^{4}\left(\left(\left|\mathbf{y}-\mathbf{y}_{d}\right|^{2}-\left|\mathbf{y}_{n, h}-\mathbf{y}_{d}\right|^{2}\right)\left(\mathbf{y}-\mathbf{y}_{d}\right), \boldsymbol{\epsilon}_{n, h}\right) \mathrm{d} t \\
& +\int_{t_{n-1}}^{t_{n}}\left\|\mathbf{y}_{n, h}-\mathbf{y}_{d}\right\|_{\mathbf{L}^{4}\left(\Omega_{h}\right)}^{4}\left(\left|\mathbf{y}_{n, h}-\mathbf{y}_{d}\right|^{2}\left(\mathbf{y}-\mathbf{y}_{n, h}\right), \boldsymbol{\epsilon}_{n, h}\right) \mathrm{d} t \equiv I_{3}^{1}+I_{3}^{2} .
\end{aligned}
$$

Then, Hölder's inequality and the embedding $\mathbf{H}^{1}(\Omega) \subset \mathbf{L}^{6}(\Omega)$ imply that

$$
\begin{aligned}
& I_{3}^{2} \leq C \int_{t_{n-1}}^{t_{n}}\left\|\mathbf{y}_{n, h}-\mathbf{y}_{d}\right\|_{\mathbf{L}^{\mathbf{6}}\left(\Omega_{h}\right)}^{6}\left\|\mathbf{y}-\mathbf{y}_{n, h}\right\|_{\mathbf{H}^{1}\left(\Omega_{h}\right)}\left\|\boldsymbol{\epsilon}_{n, h}\right\|_{\mathbf{L}^{2}\left(\Omega_{h}\right)} \mathrm{d} t \\
& \leq C \int_{t_{n-1}}^{t_{n}}\left\|\mathbf{y}-\mathbf{y}_{n, h}\right\|_{\mathbf{H}^{1}\left(\Omega_{h}\right)}^{2} \mathrm{~d} t+\int_{t_{n-1}}^{t_{n}}\left\|\boldsymbol{\epsilon}_{n, h}\right\|_{\mathbf{L}^{2}\left(\Omega_{h}\right)}^{2} \mathrm{~d} t,
\end{aligned}
$$

where constant $C$ depends on the $L^{\infty}\left(0, T ; \mathbf{L}^{6}(\Omega)\right)$ norms of $\mathbf{y}_{d}, \mathbf{y}, \mathbf{y}_{\sigma}$. Integral $I_{3}^{1}$ can be handled in a similar way.

Finally, we have the following theorem [5, Thm. 4.16].

Theorem 4.7. Suppose that (3.19) and (4.12) hold. Then, there exists a constant $C>0$ independent of $\sigma$ such that

$$
\begin{aligned}
& \left\|\overline{\mathbf{u}}-\overline{\mathbf{u}}_{\sigma}\right\|_{L^{2}\left(0, T ; \mathbf{L}^{2}\left(\Omega_{h}\right)\right)} \leq C h, \\
& \left\|\overline{\mathbf{y}}-\overline{\mathbf{y}}_{\sigma}\right\|_{L^{\infty}\left(0, T ; \mathbf{L}^{2}\left(\Omega_{h}\right)\right)}+\left\|\overline{\mathbf{y}}-\overline{\mathbf{y}}_{\sigma}\right\|_{L^{2}\left(0, T ; \mathbf{H}^{1}\left(\Omega_{h}\right)\right)} \leq C h, \\
& \left\|\overline{\boldsymbol{\varphi}}-\overline{\boldsymbol{\varphi}}_{\sigma}\right\|_{L^{\infty}\left(0, T ; \mathbf{L}^{2}\left(\Omega_{h}\right)\right)}+\left\|\overline{\boldsymbol{\varphi}}-\overline{\boldsymbol{\varphi}}_{\sigma}\right\|_{L^{2}\left(0, T ; \mathbf{H}^{1}\left(\Omega_{h}\right)\right)} \leq C h .
\end{aligned}
$$

5. Sparse controls. In the applications, we are frequently required to localize the controls in a small region of the domain. An interesting issue is to guess the region where the controls are more efficient. To answer this question we consider the following control problem:

$$
\left(\mathrm{P}_{\kappa}\right)\left\{\begin{array}{l}
\min J_{\kappa}(\mathbf{u}) \\
\mathbf{u} \in \mathcal{U}_{a d}
\end{array}\right.
$$

where

$$
J_{\kappa}(\mathbf{u})=J(\mathbf{u})+\kappa j(\mathbf{u}) \text { and } j(\mathbf{u})=\|\mathbf{u}\|_{\mathbf{L}^{1}\left(\Omega_{T}\right)}=\sum_{j=1}^{3} \int_{\Omega_{T}}\left|u_{j}(t, x)\right| d x d t,
$$

with $\kappa>0$. In this section, we assume that $-\infty<\alpha_{j}<0<\beta_{j}<+\infty, 1 \leq j \leq 3$. Thus, any admissible control can take the value 0 in some points. We will show that the solutions $\overline{\mathbf{u}}$ of $\left(\mathrm{P}_{\kappa}\right)$ are sparse controls, and the size of their supports can be monitored by $\kappa$. The bigger $\kappa$, the smaller the support of $\overline{\mathbf{u}}$.

The functional $j$ is convex and Lipschitz. Its subdifferential is defined by

$$
\partial j(\mathbf{u})=\left\{\boldsymbol{\zeta} \in \mathbf{L}^{\infty}\left(\Omega_{T}\right): \int_{\Omega_{T}} \boldsymbol{\zeta}(\mathbf{v}-\mathbf{u}) d x d t+j(\mathbf{u}) \leq j(\mathbf{v}) \quad \forall \mathbf{v} \in \mathbf{L}^{1}\left(\Omega_{T}\right)\right\} .
$$

By taking $\mathbf{v}=\mathbf{0}$ and $\mathbf{v}=2 \mathbf{u}$, respectively, we get that

$$
\int_{\Omega_{T}} \boldsymbol{\zeta} \mathbf{u} d x d t=j(\mathbf{u}), \text { and hence } \int_{\Omega_{T}} \boldsymbol{\zeta} \mathbf{v} d x d t \leq j(\mathbf{v}) \quad \forall \mathbf{v} \in \mathbf{L}^{1}\left(\Omega_{T}\right) .
$$

Copyright $@$ ㅇ by SIAM. Unauthorized reproduction of this article is prohibited. 
From here we infer

$$
\zeta_{j}(t, x) \in \operatorname{sign}\left(u_{j}(t, x)\right), \quad 1 \leq j \leq 3, \quad \text { where } \operatorname{sign}(s)=\left\{\begin{array}{cc}
\{+1\} & \text { if } s>0 \\
\{-1\} & \text { if } s<0 \\
{[-1,+1]} & \text { if } s=0
\end{array}\right.
$$

We have the following theorem, which is analogous to Theorem 3.2.

TheOREM 5.1. Under assumption (3.1), $\left(\mathrm{P}_{\kappa}\right)$ has at least one solution. Moreover, for any local solution $\overline{\mathbf{u}}$ there exist $\overline{\mathbf{y}}, \bar{\varphi} \in \mathbf{H}^{2,1}\left(\Omega_{T}\right) \cap C([0, T], \mathbf{Y}), \bar{p}, \bar{\pi} \in$ $L^{2}\left(0, T ; H^{1}(\Omega)\right)$, and $\overline{\boldsymbol{\zeta}} \in \partial j(\overline{\mathbf{u}})$ such that (3.7) and (3.8) hold, and additionally,

$$
\int_{0}^{T} \int_{\Omega}(\overline{\boldsymbol{\varphi}}+\lambda \overline{\mathbf{u}}+\kappa \overline{\boldsymbol{\zeta}})(\mathbf{u}-\overline{\mathbf{u}}) d x d t \geq 0 \quad \forall \mathbf{u} \in \mathcal{U}_{\alpha, \beta}
$$

Proof. The existence of a solution is proved in the same manner as in the proof of Theorem 3.2. Let us prove the optimality conditions. First, we observe that $\mathcal{A}$ is open and $\overline{\mathbf{u}} \in \mathcal{A}$; then for every $\mathbf{u} \in \mathcal{U}_{\alpha, \beta}$ there exists $\rho_{\mathbf{u}}>0$ such that $\overline{\mathbf{u}}+\rho(\mathbf{u}-\overline{\mathbf{u}}) \in$ $\mathcal{A} \forall 0<\rho<\rho_{\mathbf{u}}$, and hence $\overline{\mathbf{u}}+\rho(\mathbf{u}-\overline{\mathbf{u}})$ belongs to $\mathcal{U}_{a d}$. Now we use (3.3), the convexity of $j$, and the local optimality of $\overline{\mathbf{u}}$ as follows:

$$
\begin{aligned}
0 & \leq \lim _{\rho \searrow 0} \frac{J_{\kappa}(\overline{\mathbf{u}}+\rho(\mathbf{u}-\overline{\mathbf{u}}))-J_{\kappa}(\overline{\mathbf{u}})}{\rho} \leq \lim _{\rho \searrow 0} \frac{J(\overline{\mathbf{u}}+\rho(\mathbf{u}-\overline{\mathbf{u}}))-J(\overline{\mathbf{u}})}{\rho}+\kappa j(\mathbf{u})-\kappa j(\overline{\mathbf{u}}) \\
& =J^{\prime}(\overline{\mathbf{u}})(\mathbf{u}-\overline{\mathbf{u}})+\kappa j(\mathbf{u})-\kappa j(\overline{\mathbf{u}})=\int_{\Omega_{T}}(\bar{\varphi}+\lambda \overline{\mathbf{u}})(\mathbf{u}-\overline{\mathbf{u}}) d x d t+\kappa j(\mathbf{u})-\kappa j(\overline{\mathbf{u}}) .
\end{aligned}
$$

This implies that $\overline{\mathbf{u}}$ is the solution of the optimization problem

$$
\min _{\mathbf{u} \in \mathcal{U}_{\alpha, \beta}} \int_{\Omega_{T}}(\bar{\varphi}+\lambda \overline{\mathbf{u}}) \mathbf{u} d x d t+\kappa j(\mathbf{u}) .
$$

Hence, by using the subdifferential calculus for convex functions, we deduce the existence of $\overline{\boldsymbol{\zeta}} \in \partial j(\overline{\mathbf{u}})$ such that (5.3) holds.

Corollary 5.2. Suppose the assumptions of Theorem 5.1 hold, and let $(\overline{\mathbf{u}}, \overline{\boldsymbol{\varphi}}, \overline{\boldsymbol{\zeta}})$ satisfy (3.7), (3.8), and (5.3). Then the following relations hold for $1 \leq j \leq 3$ :

$$
\begin{aligned}
& \bar{u}_{j}(t, x)=\operatorname{Proj}_{\left[\alpha_{j}, \beta_{j}\right]}\left\{-\frac{1}{\lambda}\left(\bar{\varphi}_{j}(t, x)+\kappa \bar{\zeta}_{j}(t, x)\right)\right\}, \\
& \bar{u}_{j}(t, x)=0 \Leftrightarrow\left|\bar{\varphi}_{j}(t, x)\right| \leq \kappa, \\
& \bar{\zeta}_{j}(t, x)=\operatorname{Proj}_{[-1,+1]}\left(-\frac{1}{\kappa} \bar{\varphi}_{j}(t, x)\right) .
\end{aligned}
$$

Moreover, from the representation formulas (5.4) and (5.6), we have the following regularity:

$$
\overline{\mathbf{u}}, \overline{\boldsymbol{\zeta}} \in \mathbf{H}^{1}\left(\Omega_{T}\right) \cap C\left([0, T], \mathbf{H}^{1}(\Omega)\right) \cap L^{2}\left(0, T ; \mathbf{W}^{1,6}(\Omega)\right) \cap L^{2}(0, T ; \mathbf{C}(\bar{\Omega})) .
$$

Finally, $\overline{\boldsymbol{\zeta}}$ is unique for any fixed local minimum $\overline{\mathbf{u}}$.

Proof. The proof of the identity (5.4) is standard and well known in control theory. Let us prove (5.5). To this end we deduce from (5.2) and (5.4) that

$$
\begin{aligned}
& \bar{u}_{j}(t, x)=0 \Rightarrow \bar{\varphi}_{j}(t, x)+\kappa \zeta_{j}(t, x)=0 \Rightarrow\left|\bar{\varphi}_{j}(t, x)\right| \leq \kappa, \\
& \bar{u}_{j}(t, x)>0 \Rightarrow \zeta_{j}(t, x)=+1 \text { and } \bar{\varphi}_{j}(t, x)+\kappa \zeta_{j}(t, x)<0 \Rightarrow \bar{\varphi}_{j}(t, x)<-\kappa, \\
& \bar{u}_{j}(t, x)<0 \Rightarrow \zeta_{j}(t, x)=-1 \text { and } \bar{\varphi}_{j}(t, x)+\kappa \zeta_{j}(t, x)>0 \Rightarrow \bar{\varphi}_{j}(t, x)>+\kappa,
\end{aligned}
$$

Copyright $@$ by SIAM. Unauthorized reproduction of this article is prohibited. 
which proves (5.5). Finally we prove (5.6). Using (5.2), (5.4), and (5.5) we obtain

$$
\begin{aligned}
& \bar{\varphi}_{j}(t, x)>+\kappa \Rightarrow \bar{u}_{j}(t, x)<0 \Rightarrow \zeta_{j}(t, x)=-1 \Rightarrow \zeta_{j}(t, x)=\operatorname{Proj}_{[-1,+1]}\left(-\frac{1}{\kappa} \bar{\varphi}_{j}(t, x)\right), \\
& \bar{\varphi}_{j}(t, x)<-\kappa \Rightarrow \bar{u}_{j}(t, x)>0 \Rightarrow \zeta_{j}(t, x)=+1 \Rightarrow \zeta_{j}(t, x)=\operatorname{Proj}_{[-1,+1]}\left(-\frac{1}{\kappa} \bar{\varphi}_{j}(t, x)\right), \\
& \left|\bar{\varphi}_{j}(t, x)\right| \leq \kappa \Rightarrow \bar{u}_{j}(t, x)=0 \Rightarrow \bar{\varphi}_{j}(t, x)+\kappa \zeta_{j}(t, x)=0 \Rightarrow \zeta_{j}(t, x)=-\frac{1}{\kappa} \bar{\varphi}_{j}(t, x),
\end{aligned}
$$

and (5.6) follows.

Remark 5.3. Relation (5.5) shows the sparsity of the optimal controls. When $\kappa$ is increased, the support of the control is decreased. Actually, if $\kappa$ is too large, it could happen that $\overline{\mathbf{u}}(t, x)=0$. For example, if we assume that $\Gamma$ is of class $C^{3}$, $\mathbf{y}_{0}, \mathbf{y}_{\Omega} \in \mathbf{Y} \cap W_{0}^{1, p}(\Omega)$ with $p>4$, and $\mathbf{y}_{d} \in L^{q}\left(\Omega_{T}\right)$ for $q$ sufficiently large, then $\|\bar{\varphi}\|_{L^{\infty}\left(\Omega_{T}\right)} \leq M$ with $M$ independent of $\kappa$. Indeed, from the relation

$$
\frac{1}{8}\left\|\overline{\mathbf{y}}-\overline{\mathbf{y}}_{d}\right\|_{\mathbf{L}^{4}(\Omega)}^{8} \leq J_{\kappa}(\overline{\mathbf{u}}) \leq J_{\kappa}(0)=J(0)=\frac{1}{8}\left\|\mathbf{y}_{d}\right\|_{\mathbf{L}^{4}(\Omega)}^{8}
$$

and (2.5) along with the boundedness of $\mathcal{U}_{a d}$ in $L^{\infty}\left(\Omega_{T}\right)$, we infer with Solonnikov's theorem [19] that $\overline{\mathbf{y}} \in L^{p}\left(0, T ; \mathbf{W}^{2, p}(\Omega)\right) \cap \mathbf{W}^{1, p}\left(\Omega_{T}\right)$, and the estimate for $\overline{\mathbf{y}}$ in this space is independent of $\kappa$. Now, using the adjoint state equation and [19] again, we deduce that $\overline{\boldsymbol{\varphi}} \in \mathbf{W}^{1, p}\left(\Omega_{T}\right) \subset \mathbf{C}\left(\bar{\Omega}_{T}\right)$. Moreover, $\|\bar{\varphi}\|_{L^{\infty}\left(\Omega_{T}\right)}$ depends only on $\overline{\mathbf{y}}, \mathbf{y}_{d}$, and $\mathbf{y}_{\Omega}$. Hence, we get the existence of the constant $M$ independent of $\kappa$. Therefore, if we take $\kappa \geq M,(5.5)$ implies that $\overline{\mathbf{u}}=0$ is the unique solution of $\left(\mathrm{P}_{\kappa}\right)$.

Next, we establish the second order sufficient optimality conditions. To this end, first we recall that $j: \mathbf{L}^{1}\left(\Omega_{T}\right) \longrightarrow \mathbb{R}$ is convex and Lipschitz. Therefore, there exist the directional derivatives, given in this case by the formula

$$
\begin{gathered}
j^{\prime}(\mathbf{u} ; \mathbf{v})=\lim _{\rho \searrow 0} \frac{j(\mathbf{u}+\rho \mathbf{v})-j(\mathbf{u})}{\rho} \\
=\sum_{j=1}^{3}\left\{\int_{\Omega_{T, j}^{+}} v_{j} d x d t-\int_{\Omega_{T, j}^{-}} v_{j} d x d t+\int_{\Omega_{T, j}^{0}}\left|v_{j}\right| d x d t\right\}, 1 \leq j \leq 3,
\end{gathered}
$$

where $\Omega_{T, j}^{+}, \Omega_{T, j}^{-}$, and $\Omega_{T, j}^{0}$ denote the subsets of $\Omega_{T}$ where $\mathbf{u}$ takes positive, negative, or zero values, respectively. Moreover, the following properties hold:

$$
\max _{\boldsymbol{\zeta} \in \partial j(\mathbf{u})} \int_{\Omega_{T}} \boldsymbol{\zeta} \mathbf{v} d x d t=j^{\prime}(\mathbf{u} ; \mathbf{v}) \leq \frac{j(\mathbf{u}+\rho \mathbf{v})-j(\mathbf{u})}{\rho} \leq j(\mathbf{u}+\mathbf{v})-j(\mathbf{u})
$$

$\forall \rho \in(0,1)$. The reader is referred to [2, sect. 2.4.3], [3, Chap. 4], or [8, Chap. 2] for these issues.

Now, we fix an element $\overline{\mathbf{u}} \in \mathcal{U}_{a d}$ satisfying the first order optimality conditions established in Theorem 5.1. We associate to $\overline{\mathbf{u}}$ the cone of critical directions defined by

$$
C_{\overline{\mathbf{u}}}=\left\{\mathbf{v} \in L^{2}\left(0, T ; \mathbf{L}^{2}(\Omega)\right): \mathbf{v} \text { satisfies }(5.10) \text { and } J^{\prime}(\overline{\mathbf{u}}) \mathbf{v}+\kappa j^{\prime}(\overline{\mathbf{u}} ; \mathbf{v})=0\right\},
$$

with

$$
\left\{\begin{array}{l}
v_{j}(t, x) \geq 0 \quad \text { if } \bar{u}_{j}(t, x)=\alpha_{j}, \\
v_{j}(t, x) \leq 0 \quad \text { if } \bar{u}_{j}(t, x)=\beta_{j}, \quad 1 \leq j \leq 3
\end{array}\right.
$$

Copyright $@$ by SIAM. Unauthorized reproduction of this article is prohibited. 
Before stating the second order optimality conditions, let us give some properties that will be useful later.

Proposition 5.4. Let $(\overline{\mathbf{u}}, \overline{\boldsymbol{\zeta}}) \in \mathcal{U}_{a d} \times \partial j(\overline{\mathbf{u}})$ satisfy the optimality system (3.7), (3.8), and (5.3) along with the state $\overline{\mathbf{y}}$ and adjoint state $\bar{\varphi}$. Let $v \in L^{2}\left(0, T ; \mathbf{L}^{2}(\Omega)\right)$ fulfill (5.10). Then

$$
J^{\prime}(\overline{\mathbf{u}}) \mathbf{v}+\kappa j^{\prime}(\overline{\mathbf{u}} ; \mathbf{v}) \geq J^{\prime}(\overline{\mathbf{u}}) \mathbf{v}+\kappa \int_{\Omega_{T}} \overline{\boldsymbol{\zeta}} \mathbf{v} d x d t \geq 0 .
$$

Moreover, if $\mathbf{v} \in C_{\overline{\mathbf{u}}}$, then

$$
J^{\prime}(\overline{\mathbf{u}}) \mathbf{v}+\kappa \int_{\Omega_{T}} \overline{\boldsymbol{\zeta}} \mathbf{v} d x d t=0 \text { and } j^{\prime}(\overline{\mathbf{u}} ; \mathbf{v})=\int_{\Omega_{T}} \overline{\boldsymbol{\zeta}} \mathbf{v} d x d t .
$$

Proposition 5.5. The set $C_{\overline{\mathbf{u}}}$ is a closed, convex cone in $L^{2}\left(0, T ; \mathbf{L}^{2}(\Omega)\right)$.

The reader is referred to [6, Lem. 3.5 and Prop. 3.4] for the proof of these propositions.

Let us define $\overline{\mathbf{d}}(t, x)=\bar{\varphi}(t, x)+\lambda \overline{\mathbf{u}}(t, x)+\kappa \overline{\boldsymbol{\zeta}}(t, x)$. From (5.3), we deduce in the usual way for $1 \leq j \leq 3$ that

$$
\left\{\begin{array}{cl}
\bar{u}_{j}(t, x)=\alpha_{j} & \Rightarrow \bar{d}_{j}(t, x) \geq 0 \\
\bar{u}_{j}(t, x)=\beta_{j} & \Rightarrow \bar{d}_{j}(t, x) \leq 0, \quad 1 \leq j \leq 3 \\
\alpha_{j}<\bar{u}_{j}(t, x)<\beta_{j} & \Rightarrow \bar{d}_{j}(t, x)=0
\end{array}\right.
$$

and

$$
\left\{\begin{array}{ll}
\bar{d}_{j}(t, x)>0 & \Rightarrow \bar{u}_{j}(t, x)=\alpha_{j}, \quad \\
\bar{d}_{j}(t, x)<0 & \Rightarrow \bar{u}_{j}(t, x)=\beta_{j},
\end{array} \quad 1 \leq j \leq 3\right.
$$

Now, from (5.12) we infer

$$
\int_{\Omega_{T}} \overline{\mathbf{d}} \mathbf{v} d x d t=J^{\prime}(\overline{\mathbf{u}}) \mathbf{v}+\kappa \int_{\Omega_{T}} \overline{\boldsymbol{\zeta}} \mathbf{v} d x d t=0 \quad \forall \mathbf{v} \in C_{\overline{\mathbf{u}}} .
$$

This identity, along with (5.13) and (5.14), implies

$$
\bar{d}_{j}(t, x) v_{j}(t, x)=\left|\bar{d}_{j}(t, x) v_{j}(t, x)\right|=0, \quad 1 \leq j \leq 3, \forall \mathbf{v} \in C_{\overline{\mathbf{u}}} .
$$

The following theorem states the second order optimality conditions.

THEOREM 5.6. The following statements hold:

(i) Let $\overline{\mathbf{u}}$ be a local minimum of $\left(\mathrm{P}_{\kappa}\right)$; then $J^{\prime \prime}(\overline{\mathbf{u}}) \mathbf{v}^{2} \geq 0 \forall \mathbf{v} \in C_{\overline{\mathbf{u}}}$.

(ii) Let $(\overline{\mathbf{u}}, \overline{\boldsymbol{\zeta}}) \in \mathcal{U}_{a d} \times \partial j(\overline{\mathbf{u}})$ satisfy (5.3). Furthermore, let us assume that

$$
J^{\prime \prime}(\overline{\mathbf{u}}) \mathbf{v}^{2}>0 \quad \forall \mathbf{v} \in C_{\overline{\mathbf{u}}} \backslash\{0\} ;
$$

then there exist $\varepsilon>0$ and $\delta>0$ such that

$$
J_{\kappa}(\overline{\mathbf{u}})+\frac{\delta}{2}\|\mathbf{u}-\overline{\mathbf{u}}\|_{L^{2}\left(0, T ; \mathbf{L}^{2}(\Omega)\right)}^{2} \leq J_{\kappa}(\mathbf{u}) \quad \forall \mathbf{u} \in B_{\varepsilon}(\overline{\mathbf{u}}) \cap \mathcal{U}_{\alpha, \beta},
$$

where $B_{\varepsilon}(\overline{\mathbf{u}})$ is the $L^{2}\left(0, T ; \mathbf{L}^{2}(\Omega)\right)$-ball of center $\overline{\mathbf{u}}$ and radius $\varepsilon$.

The proof of this theorem is the same as the proofs of Theorem 3.7 and 3.9 of [6]. Indeed, it is enough to use (5.13)-(5.15), instead of the relations (3.11) and (3.12) of [6], and select $\varepsilon>0$ such that $B_{\varepsilon}(\overline{\mathbf{u}}) \subset \mathcal{A}$.

Corollary 5.7. Let $(\overline{\mathbf{u}}, \overline{\boldsymbol{\zeta}})$ be as in the previous theorem, and assume that (5.16) is fulfilled. Then, the following inequality holds:

$$
J^{\prime \prime}(\overline{\mathbf{u}}) \mathbf{v}^{2} \geq \delta\|\mathbf{v}\|_{L^{2}\left(0, T ; \mathbf{L}^{2}(\Omega)\right)}^{2} \quad \forall \mathbf{v} \in C_{\overline{\mathbf{u}}} .
$$

Copyright (c) by SIAM. Unauthorized reproduction of this article is prohibited. 
Proof. From (5.17) we infer that $\overline{\mathbf{u}}$ is a local solution of the problem

$$
\left\{\begin{array}{l}
\min I(\mathbf{u})=J_{\kappa}(\mathbf{u})-\frac{\delta}{2}\|\mathbf{u}-\overline{\mathbf{u}}\|_{L^{2}\left(0, T ; \mathbf{L}^{2}(\Omega)\right)}^{2}, \\
\mathbf{u} \in \mathcal{U}_{a d}
\end{array}\right.
$$

Hence, Theorem 5.6(i) implies

$$
0 \leq I^{\prime \prime}(\overline{\mathbf{u}}) \mathbf{v}^{2}=J^{\prime \prime}(\overline{\mathbf{u}}) \mathbf{v}^{2}-\delta\|\mathbf{v}\|_{L^{2}\left(0, T ; \mathbf{L}^{2}(\Omega)\right)}^{2} \quad \forall \mathbf{v} \in C_{\overline{\mathbf{u}}},
$$

which concludes the proof.

5.1. Numerical approximation of $\left(\mathrm{P}_{\kappa}\right)$ and error estimates. Hereafter, we assume that $\Omega$ is convex. Following the same scheme as for $\left(\mathrm{P}_{\sigma}\right)$, we define the discrete approximation of $\left(\mathrm{P}_{\kappa}\right)$ by

$$
\left(\mathrm{P}_{\kappa \sigma}\right)\left\{\begin{array}{l}
\min \mathcal{J}_{\kappa \sigma}\left(\mathbf{u}_{\sigma}, \mathbf{y}_{\sigma}\right) \\
\left(\mathbf{u}_{\sigma}, \mathbf{y}_{\sigma}\right) \in \mathcal{U}_{\sigma, a d} \times \mathcal{Y}_{\sigma} \text { satisfy }(4.9),
\end{array}\right.
$$

where

$$
\mathcal{J}_{\kappa \sigma}\left(\mathbf{u}_{\sigma}, \mathbf{y}_{\sigma}\right)=\mathcal{J}_{\sigma}\left(\mathbf{u}_{\sigma}, \mathbf{y}_{\sigma}\right)+\kappa j_{\sigma}\left(\mathbf{u}_{\sigma}\right)=\mathcal{J}_{\sigma}\left(\mathbf{u}_{\sigma}, \mathbf{y}_{\sigma}\right)+\kappa \sum_{j=1}^{3} \int_{0}^{T} \int_{\Omega_{h}}\left|u_{j}(t, x)\right| d x d t
$$

Theorems 4.2 and 4.3 can be proved for $\left(\mathrm{P}_{\kappa \sigma}\right)$ in the same manner that they were proved in section 4 . From now on, $\overline{\mathbf{u}}$ will denote a local (or global) minimum of $\left(\mathrm{P}_{\kappa}\right)$, with associated state $\overline{\mathbf{y}}$ and adjoint state $\bar{\varphi}$, and $\bar{\zeta} \in \partial j(\overline{\mathbf{u}})$ is the unique element such that $(\overline{\mathbf{u}}, \overline{\mathbf{y}}, \overline{\boldsymbol{\varphi}}, \overline{\boldsymbol{\zeta}})$ satisfies the first order optimality conditions. We also assume that $\overline{\mathbf{u}}$ satisfies the second order sufficient condition (5.16). As in section 4, we take a sequence of local or global solutions $\left\{\left(\overline{\mathbf{u}}_{\sigma}, \overline{\mathbf{y}}_{\sigma}\right)\right\}_{\sigma}$ of $\left(\mathrm{P}_{\kappa \sigma}\right)$ satisfying (4.15) and (4.16). Following section 4, we take $\sigma_{0}>0$ and $\varepsilon>0$ such that $B_{\varepsilon}(\overline{\mathbf{u}}) \subset \mathcal{A}$ and (4.9) has a unique solution for every $\mathbf{u} \in B_{\varepsilon}(\overline{\mathbf{u}})$, and we define

$$
J_{\kappa \sigma}: B_{\varepsilon}(\overline{\mathbf{u}}) \longrightarrow \mathbb{R} \quad \text { by } \quad J_{\kappa \sigma}(\mathbf{u})=\mathcal{J}_{\kappa \sigma}\left(\mathbf{u}, \mathbf{y}_{\sigma}(\mathbf{u})\right) .
$$

Moreover, $\overline{\mathbf{u}}$ and $\overline{\mathbf{u}}_{\sigma}$ are global minima of $J_{\kappa}$ and $J_{\kappa \sigma}$, respectively, in $B_{\varepsilon}(\overline{\mathbf{u}})$. In addition, we take $\varepsilon$ small enough so that (5.17) holds for this $\varepsilon$ and some $\delta>0$.

Now, for every $\overline{\mathbf{u}}_{\sigma}$ with $|\sigma|<\sigma_{0}$, Theorem 4.4 and Corollary 4.5 hold with the only following change: (4.25) is replaced by

$$
\int_{0}^{T} \int_{\Omega_{h}}\left(\overline{\boldsymbol{\varphi}}_{\sigma}+\lambda \overline{\mathbf{u}}_{\sigma}+\kappa \overline{\boldsymbol{\zeta}}_{\sigma}\right)\left(\mathbf{u}_{\sigma}-\overline{\mathbf{u}}_{\sigma}\right) d x d t \geq 0 \quad \forall \mathbf{u}_{\sigma} \in \mathcal{U}_{\sigma, a d}
$$

where $\overline{\boldsymbol{\zeta}}_{\sigma} \in \partial j_{\sigma}\left(\overline{\mathbf{u}}_{\sigma}\right)$. This inequality can be proved in the same way as (5.3). From $\overline{\boldsymbol{\zeta}}_{\sigma} \in \partial j_{\sigma}\left(\overline{\mathbf{u}}_{\sigma}\right), j_{\sigma}: \mathcal{U}_{\sigma} \longrightarrow \mathbb{R}$, it is easy to deduce that

$$
\overline{\boldsymbol{\zeta}}_{\sigma}=\sum_{n=1}^{N_{\tau}} \sum_{K \in \mathcal{K}_{h}} \bar{\zeta}_{n, K} \chi_{n} \chi_{K} \quad \text { with }\left\{\begin{array}{cc}
\bar{\zeta}_{n, K}=+1 & \text { if } \bar{u}_{n, K}>0, \\
\bar{\zeta}_{n, K}=-1 & \text { if } \bar{u}_{n, K}<0, \\
\bar{\zeta}_{n, K} \in[-1,+1] & \text { if } \bar{u}_{n, K}=0,
\end{array}\right.
$$

where $\left\{\bar{u}_{n, K}\right\}$ denote the coefficients of $\overline{\mathbf{u}}_{\sigma}$,

$$
\overline{\mathbf{u}}_{\sigma}=\sum_{n=1}^{N_{\tau}} \sum_{K \in \mathcal{K}_{h}} \overline{\mathbf{u}}_{n, K} \chi_{n} \chi_{K}
$$

Copyright (c) by SIAM. Unauthorized reproduction of this article is prohibited. 
Inequality (5.18) can be written

$$
\sum_{n=1}^{N_{\tau}} \sum_{K \in \mathcal{K}_{h}}\left(\int_{t_{n-1}}^{t_{n}} \int_{K} \bar{\varphi}_{\sigma}(t, x) \mathrm{d} x d t+\tau_{n}|K|\left[\lambda \bar{u}_{n, K}+\kappa \bar{\zeta}_{n, K}\right]\right)\left(u_{n, K}-\bar{u}_{n, k}\right) \geq 0
$$

$\forall u_{n, k} \in[\alpha, \beta]$ or, equivalently,

$$
\sum_{n=1}^{N_{\tau}} \sum_{K \in \mathcal{K}_{h}}\left(\tau_{n} \int_{K} \bar{\varphi}_{j, n, h}(x) \mathrm{d} x+\tau_{n}|K|\left[\lambda \bar{u}_{j, n, K}+\kappa \bar{\zeta}_{j, n, K}\right]\right)\left(u_{j, n, K}-\bar{u}_{j, n, k}\right) \geq 0
$$

$\forall u_{j, n, k} \in\left[\alpha_{j}, \beta_{j}\right]$ and $1 \leq j \leq 3$. Now, arguing as in Corollary 5.2, we have the following result.

Corollary 5.8. Let $\left(\overline{\mathbf{u}}_{\sigma}, \overline{\mathbf{y}}_{\sigma}, \overline{\boldsymbol{\varphi}}_{\sigma}, \overline{\boldsymbol{\zeta}}_{\sigma}\right)$ satisfy the discrete optimality system for $\left(\mathrm{P}_{\kappa \sigma}\right)$. Then the following relations hold for $K \in \mathcal{K}_{h}$ and for $1 \leq j \leq 3$ :

$$
\begin{aligned}
& \bar{u}_{j, n, K}=\operatorname{Proj}_{\left[\alpha_{j}, \beta_{j}\right]}\left\{-\frac{1}{\lambda}\left(\frac{1}{|K|} \int_{K} \bar{\varphi}_{j, n, h}(x) d x+\kappa \bar{\zeta}_{j, n, K}\right)\right\}, \\
& \bar{u}_{j, n, K}=0 \Leftrightarrow\left|\frac{1}{|K|} \int_{K} \bar{\varphi}_{j, n, h}(x) d x\right| \leq \kappa, \\
& \bar{\zeta}_{j, n, K}=\operatorname{Proj}_{[-1,+1]}\left(-\frac{1}{\kappa|K|} \int_{K} \bar{\varphi}_{j, n, h}(x) d x\right) .
\end{aligned}
$$

Moreover, from the representation formula (5.22) it follows that $\overline{\boldsymbol{\zeta}}_{\sigma}$ is unique for any fixed local minimum $\overline{\mathbf{u}}_{\sigma}$.

The following convergence properties will be used later:

$$
\lim _{|\sigma| \rightarrow 0}\left\{\left\|\overline{\boldsymbol{\varphi}}_{\sigma}-\overline{\boldsymbol{\varphi}}\right\|_{L^{2}\left(0, T ; \mathbf{L}^{2}\left(\Omega_{h}\right)\right)}+\left\|\overline{\boldsymbol{\zeta}}_{\sigma}-\overline{\boldsymbol{\zeta}}\right\|_{L^{2}\left(0, T ; \mathbf{L}^{2}\left(\Omega_{h}\right)\right)}\right\}=0 .
$$

The convergence for the adjoint states follows from (4.26). From the representation formulas (5.6) and (5.22) we infer the convergence for $\overline{\boldsymbol{\zeta}}_{\sigma}-\overline{\boldsymbol{\zeta}}$.

We finish the paper by proving that Theorem 4.7 holds for problem $\left(\mathrm{P}_{\kappa}\right)$.

THEOREM 5.9. Under the previous notation, and assuming that (4.12) holds and $\bar{u}$ satisfies the sufficient second order condition (5.16), the error estimates (4.27)(4.29) remain valid. Additionally, we have the estimate

$$
\left\|\overline{\boldsymbol{\zeta}}-\overline{\boldsymbol{\zeta}}_{\sigma}\right\|_{L^{2}\left(0, T ; \mathbf{L}^{2}\left(\Omega_{h}\right)\right)} \leq C h .
$$

Proof. We will prove (4.27). The estimates (4.28) and (4.29) are an immediate consequence of (4.13), (4.26), and (4.27). The estimate (5.24) follows from (4.29) and the representation formulas (5.6) and (5.22).

Let us extend $\overline{\mathbf{u}}_{\sigma}$ to $\Omega_{T}$ by setting $\overline{\mathbf{u}}_{\sigma}(t, x)=\overline{\mathbf{u}}(t, x)$ for $x \in \Omega \backslash \Omega_{h}$. Since $\overline{\mathbf{u}}_{\sigma} \rightarrow \overline{\mathbf{u}}$ in $L^{2}\left(0, T ; \mathbf{L}^{2}(\Omega)\right)$, there exists $\sigma_{\varepsilon} \in\left(0, \sigma_{0}\right)$ such that $\overline{\mathbf{u}}_{\sigma} \in B_{\varepsilon}(\overline{\mathbf{u}})$ for every $|\sigma|<\sigma_{\varepsilon}$. Hence, we obviously have that $\overline{\mathbf{u}}_{\sigma} \in \mathcal{U}_{a d}$ for $|\sigma|<\sigma_{\varepsilon}$. We proceed by contradiction and assume that for any constant $C>0$ and any $\sigma_{0}>0$ there exists $\sigma$ with $|\sigma|<\sigma_{0}$ such that

$$
\left\|\overline{\mathbf{u}}_{\sigma}-\overline{\mathbf{u}}\right\|_{L^{2}\left(0, T ; \mathbf{L}^{2}\left(\Omega_{h}\right)\right)}>C h .
$$

This implies that

$$
\liminf _{|\sigma| \rightarrow 0} \frac{h}{\left\|\overline{\mathbf{u}}_{\sigma}-\overline{\mathbf{u}}\right\|_{L^{2}\left(0, T ; \mathbf{L}^{2}\left(\Omega_{h}\right)\right)}}=0 .
$$

Copyright $@$ ㅇ by SIAM. Unauthorized reproduction of this article is prohibited. 
We denote by $\left\{\overline{\mathbf{u}}_{\sigma}\right\}_{\sigma}$ a sequence satisfying the above property. We will see that this is not possible.

Let us define $\mathbf{u}_{\sigma}$ as the $L^{2}\left(0, T ; \mathbf{L}^{2}(\Omega)\right)$-projection of $\overline{\mathbf{u}}$ on $\mathcal{U}_{\sigma}$, which is given by

$$
\mathbf{u}_{\sigma}=\sum_{n=1}^{N_{\tau}} \sum_{K \in \mathcal{K}_{h}} \mathbf{u}_{n, K} \chi_{n} \chi_{K}, \text { with } \mathbf{u}_{n, K}=\frac{1}{|K| \tau_{n}} \int_{t_{n-1}}^{t_{n}} \int_{K} \overline{\mathbf{u}}(t, x) d x d t .
$$

We also extend $\mathbf{u}_{\sigma}$ to $\Omega_{T}$ by taking $\mathbf{u}_{\sigma}(t, x)=\overline{\mathbf{u}}(t, x)$ for $x \in \Omega \backslash \Omega_{h}$. From the convergence of the projections, $\mathbf{u}_{\sigma} \rightarrow \overline{\mathbf{u}}$ in $L^{2}\left(0, T ; \mathbf{L}^{2}(\Omega)\right)$, we deduce that $\mathbf{u}_{\sigma} \in \mathcal{A}$ for every $|\sigma|<\sigma_{\varepsilon}$, redefining a smaller $\sigma_{\varepsilon}$ if necessary. Moreover, it is obvious that $\mathbf{u}_{\sigma}(t, x) \in[\alpha, \beta]$ for almost all $(t, x) \in \Omega_{h} \times(0, T)$, and hence $\mathbf{u}_{\sigma} \in \mathcal{U}_{\sigma, a d}$. We also have the following properties enjoyed by $\mathbf{u}_{\sigma}$ :

$$
\left\|\mathbf{u}_{\sigma}\right\|_{L^{2}\left(0, T ; \mathbf{L}^{2}\left(\Omega_{h}\right)\right)}^{2}=\left(\mathbf{u}_{\sigma}, \mathbf{u}_{\sigma}\right)=\left(\overline{\mathbf{u}}, \mathbf{u}_{\sigma}\right) \leq\|\overline{\mathbf{u}}\|_{L^{2}\left(0, T ; \mathbf{L}^{2}\left(\Omega_{h}\right)\right)}\left\|\mathbf{u}_{\sigma}\right\|_{L^{2}\left(0, T ; \mathbf{L}^{2}\left(\Omega_{h}\right)\right),}
$$

and hence,

$$
\left\|\mathbf{u}_{\sigma}\right\|_{L^{2}\left(0, T ; \mathbf{L}^{2}\left(\Omega_{h}\right)\right)} \leq\|\overline{\mathbf{u}}\|_{L^{2}\left(0, T ; \mathbf{L}^{2}\left(\Omega_{h}\right)\right)} .
$$

From the representation formula for $\mathbf{u}_{\sigma}$ written above, we infer

$$
\begin{gathered}
\left\|\mathbf{u}_{\sigma}\right\|_{L^{1}\left(0, T ; \mathbf{L}^{1}\left(\Omega_{h}\right)\right)} \leq \sum_{n=1}^{N_{\tau}} \sum_{K \in \mathcal{K}_{h}}\left|\mathbf{u}_{n, K}\right| \tau_{n}|K| \\
=\sum_{n=1}^{N_{\tau}} \sum_{K \in \mathcal{K}_{h}}\left|\int_{t_{n-1}}^{t_{n}} \int_{K} \overline{\mathbf{u}}(t, x) d x d t\right| \leq\|\overline{\mathbf{u}}\|_{L^{1}\left(0, T ; \mathbf{L}^{1}\left(\Omega_{h}\right)\right) .}
\end{gathered}
$$

The inequalities (5.26) and (5.27) are also valid in $\Omega$ because $\mathbf{u}_{\sigma}$ and $\overline{\mathbf{u}}$ coincide outside $\Omega_{h}$.

Now, using (5.18) and $\overline{\boldsymbol{\zeta}}_{\sigma} \in \partial j_{\sigma}\left(\overline{\mathbf{u}}_{\sigma}\right)$, we get

$$
\begin{gathered}
0 \leq J_{\sigma}^{\prime}\left(\overline{\mathbf{u}}_{\sigma}\right)\left(\mathbf{u}_{\sigma}-\overline{\mathbf{u}}_{\sigma}\right)+\kappa j_{\sigma}\left(\mathbf{u}_{\sigma}\right)-\kappa j_{\sigma}\left(\overline{\mathbf{u}}_{\sigma}\right) \\
=J^{\prime}\left(\overline{\mathbf{u}}_{\sigma}\right)\left(\overline{\mathbf{u}}-\overline{\mathbf{u}}_{\sigma}\right)+J^{\prime}\left(\overline{\mathbf{u}}_{\sigma}\right)\left(\mathbf{u}_{\sigma}-\overline{\mathbf{u}}\right)+\left[J_{\sigma}^{\prime}\left(\overline{\mathbf{u}}_{\sigma}\right)-J^{\prime}\left(\overline{\mathbf{u}}_{\sigma}\right)\right]\left(\mathbf{u}_{\sigma}-\overline{\mathbf{u}}_{\sigma}\right)+\kappa j\left(\mathbf{u}_{\sigma}\right)-\kappa j\left(\overline{\mathbf{u}}_{\sigma}\right),
\end{gathered}
$$

where we have used that $\overline{\mathbf{u}}_{\sigma}(t, x)=\mathbf{u}_{\sigma}(t, x)=\overline{\mathbf{u}}(t, x)$ for $(t, x) \in\left(\Omega \backslash \Omega_{h}\right) \times(0, T)$. Inserting (5.27) in the above inequality, we deduce

$$
\begin{gathered}
0 \leq J^{\prime}\left(\overline{\mathbf{u}}_{\sigma}\right)\left(\overline{\mathbf{u}}-\overline{\mathbf{u}}_{\sigma}\right)+J^{\prime}(\overline{\mathbf{u}})\left(\mathbf{u}_{\sigma}-\overline{\mathbf{u}}\right)+\left[J^{\prime}\left(\overline{\mathbf{u}}_{\sigma}\right)-J^{\prime}(\overline{\mathbf{u}})\right]\left(\mathbf{u}_{\sigma}-\overline{\mathbf{u}}\right) \\
+\left[J_{\sigma}^{\prime}\left(\overline{\mathbf{u}}_{\sigma}\right)-J^{\prime}\left(\overline{\mathbf{u}}_{\sigma}\right)\right]\left(\mathbf{u}_{\sigma}-\overline{\mathbf{u}}_{\sigma}\right)+\kappa j(\overline{\mathbf{u}})-\kappa j\left(\overline{\mathbf{u}}_{\sigma}\right) .
\end{gathered}
$$

On the other hand, (5.3) implies that

$$
0 \leq J^{\prime}(\overline{\mathbf{u}})\left(\overline{\mathbf{u}}_{\sigma}-\overline{\mathbf{u}}\right)+\kappa j\left(\overline{\mathbf{u}}_{\sigma}\right)-\kappa j(\overline{\mathbf{u}}) .
$$

Adding the last two inequalities, we obtain

$$
\begin{gathered}
{\left[J^{\prime}\left(\overline{\mathbf{u}}_{\sigma}\right)-J^{\prime}(\overline{\mathbf{u}})\right]\left(\overline{\mathbf{u}}_{\sigma}-\overline{\mathbf{u}}\right)} \\
(5.28) \leq J^{\prime}(\overline{\mathbf{u}})\left(\mathbf{u}_{\sigma}-\overline{\mathbf{u}}\right)+\left[J^{\prime}\left(\overline{\mathbf{u}}_{\sigma}\right)-J^{\prime}(\overline{\mathbf{u}})\right]\left(\mathbf{u}_{\sigma}-\overline{\mathbf{u}}\right)+\left[J_{\sigma}^{\prime}\left(\overline{\mathbf{u}}_{\sigma}\right)-J^{\prime}\left(\overline{\mathbf{u}}_{\sigma}\right)\right]\left(\mathbf{u}_{\sigma}-\overline{\mathbf{u}}_{\sigma}\right) .
\end{gathered}
$$

Let us estimate the right-hand side of the above inequality. From (3.3) and (5.27) it follows that

$$
\begin{gathered}
\left|J^{\prime}(\overline{\mathbf{u}})\left(\mathbf{u}_{\sigma}-\overline{\mathbf{u}}\right)\right| \leq \int_{0}^{T} \int_{\Omega}|\overline{\boldsymbol{\varphi}}+\lambda \overline{\mathbf{u}}|\left|\mathbf{u}_{\sigma}-\overline{\mathbf{u}}\right| d x d t \\
\leq C\|\overline{\boldsymbol{\varphi}}+\lambda \overline{\mathbf{u}}\|_{H^{1}\left(\Omega_{T}\right)}\left\|\mathbf{u}_{\sigma}-\overline{\mathbf{u}}\right\|_{H^{1}\left(\Omega_{T}\right)^{*}} \leq C h^{2}
\end{gathered}
$$

Copyright $@$ by SIAM. Unauthorized reproduction of this article is prohibited. 
see $[5$, Lem. 4.17] for the last inequality.

For the second term, we use the mean value theorem to obtain

$$
\begin{gathered}
\left|\left[J^{\prime}\left(\overline{\mathbf{u}}_{\sigma}\right)-J^{\prime}(\overline{\mathbf{u}})\right]\left(\mathbf{u}_{\sigma}-\overline{\mathbf{u}}\right)\right| \leq C\left\|\overline{\mathbf{u}}_{\sigma}-\overline{\mathbf{u}}\right\|_{L^{2}\left(0, T ; \mathbf{L}^{2}\left(\Omega_{h}\right)\right)}\left\|\mathbf{u}_{\sigma}-\overline{\mathbf{u}}\right\|_{L^{2}\left(0, T ; \mathbf{L}^{2}\left(\Omega_{h}\right)\right)} \\
\leq C\left\|\overline{\mathbf{u}}_{\sigma}-\overline{\mathbf{u}}\right\|_{L^{2}\left(0, T ; \mathbf{L}^{2}\left(\Omega_{h}\right)\right)} h .
\end{gathered}
$$

To estimate the third term, we consider the continuous and discrete adjoint states $\overline{\boldsymbol{\varphi}}^{\sigma}$ and $\overline{\boldsymbol{\varphi}}_{\sigma}$ associated to $\overline{\mathbf{u}}_{\sigma}$. By (4.26) we have that

$$
\begin{gathered}
\left|\left[J_{\sigma}^{\prime}\left(\overline{\mathbf{u}}_{\sigma}\right)-J^{\prime}\left(\overline{\mathbf{u}}_{\sigma}\right)\right]\left(\mathbf{u}_{\sigma}-\overline{\mathbf{u}}_{\sigma}\right)\right| \leq \int_{0}^{T} \int_{\Omega_{h}}\left|\bar{\varphi}_{\sigma}-\bar{\varphi}^{\sigma}\right|\left|\mathbf{u}_{\sigma}-\overline{\mathbf{u}}_{\sigma}\right| d x d t \\
\leq\left\|\overline{\boldsymbol{\varphi}}_{\sigma}-\overline{\boldsymbol{\varphi}}^{\sigma}\right\|_{L^{2}\left(0, T ; \mathbf{L}^{2}\left(\Omega_{h}\right)\right)}\left\|\mathbf{u}_{\sigma}-\overline{\mathbf{u}}_{\sigma}\right\|_{L^{2}\left(0, T ; \mathbf{L}^{2}\left(\Omega_{h}\right)\right)} \\
\leq C h\left(\left\|\mathbf{u}_{\sigma}-\overline{\mathbf{u}}\right\|_{L^{2}\left(0, T ; \mathbf{L}^{2}\left(\Omega_{h}\right)\right)}+\left\|\overline{\mathbf{u}}-\overline{\mathbf{u}}_{\sigma}\right\|_{L^{2}\left(0, T ; \mathbf{L}^{2}\left(\Omega_{h}\right)\right)}\right) \\
\leq C\left(h^{2}+\left\|\overline{\mathbf{u}}-\overline{\mathbf{u}}_{\sigma}\right\|_{L^{2}\left(0, T ; \mathbf{L}^{2}\left(\Omega_{h}\right)\right)} h\right) .
\end{gathered}
$$

Combining the estimates (5.28)-(5.31) and the following lemma, we deduce (4.27), which contradicts (5.25) as desired.

Lemma 5.10. There exist $\sigma_{0}>0$ and $\delta_{0}>0$ such that

$$
\left[J^{\prime}\left(\overline{\mathbf{u}}_{\sigma}\right)-J^{\prime}(\overline{\mathbf{u}})\right]\left(\overline{\mathbf{u}}_{\sigma}-\overline{\mathbf{u}}\right) \geq \delta_{0}\left\|\overline{\mathbf{u}}_{\sigma}-\overline{\mathbf{u}}\right\|_{L^{2}\left(0, T ; \mathbf{L}^{2}\left(\Omega_{h}\right)\right)}^{2} \quad \forall|\sigma| \leq \sigma_{0} .
$$

Proof. Let us define

$$
\mathbf{v}_{\sigma}=\frac{\overline{\mathbf{u}}_{\sigma}-\overline{\mathbf{u}}}{\left\|\overline{\mathbf{u}}_{\sigma}-\overline{\mathbf{u}}\right\|_{L^{2}\left(0, T ; \mathbf{L}^{2}(\Omega)\right)}} .
$$

Using the mean value theorem, we get

$$
\left[J^{\prime}\left(\overline{\mathbf{u}}_{\sigma}\right)-J^{\prime}(\overline{\mathbf{u}})\right]\left(\overline{\mathbf{u}}_{\sigma}-\overline{\mathbf{u}}\right)=J^{\prime \prime}\left(\mathbf{u}_{\theta, \sigma}\right)\left(\overline{\mathbf{u}}_{\sigma}-\overline{\mathbf{u}}\right)^{2},
$$

where $\mathbf{u}_{\theta, \sigma}=\overline{\mathbf{u}}+\theta_{\sigma}\left(\overline{\mathbf{u}}_{\sigma}-\overline{\mathbf{u}}\right)$ with $\theta_{\sigma} \in(0,1)$. It is immediate that $\left\{J^{\prime \prime}\left(\mathbf{u}_{\theta, \sigma}\right) \mathbf{v}_{\sigma}^{2}\right\}_{\sigma}$ is a bounded sequence of real numbers. Let us take a subsequence $\left\{\mathbf{v}_{\sigma^{\prime}}\right\}_{\sigma^{\prime}}$ of $\left\{\mathbf{v}_{\sigma}\right\}_{\sigma}$ such that

$$
\liminf _{\sigma \rightarrow 0} J^{\prime \prime}\left(\mathbf{u}_{\theta, \sigma}\right) \mathbf{v}_{\sigma}^{2}=\lim _{\sigma^{\prime} \rightarrow 0} J^{\prime \prime}\left(\mathbf{u}_{\theta, \sigma^{\prime}}\right) \mathbf{v}_{\sigma^{\prime}}^{2}=l \in \mathbb{R} .
$$

We will prove that $l>0$. To this end, we take a new subsequence of $\left\{\mathbf{v}_{\sigma^{\prime}}\right\}_{\sigma^{\prime}},\left\{\mathbf{v}_{\sigma^{\prime \prime}}\right\}_{\sigma^{\prime \prime}}$ such that $\mathbf{v}_{\sigma^{\prime \prime}} \rightarrow \mathbf{v}$ in $L^{2}\left(0, T ; \mathbf{L}^{2}(\Omega)\right)$. In what follows, we simplify the notation by denoting this sequence simply by $\left\{\mathbf{v}_{\sigma}\right\}_{\sigma}$. Then we have

$$
\lim _{\sigma \rightarrow 0} J^{\prime \prime}\left(\mathbf{u}_{\theta, \sigma}\right) \mathbf{v}_{\sigma}^{2}=l, \quad\left\|\mathbf{v}_{\sigma}\right\|_{L^{2}\left(0, T ; \mathbf{L}^{2}(\Omega)\right)}=1, \quad \text { and } \mathbf{v}_{\sigma} \rightarrow \mathbf{v} \text { in } L^{2}\left(0, T ; \mathbf{L}^{2}(\Omega)\right) .
$$

We will distinguish two cases.

Case I: $\mathbf{v}=0$. According to (3.5), $J^{\prime \prime}\left(\mathbf{u}_{\theta, \sigma}\right) \mathbf{v}_{\sigma}^{2}=$ "something converging to $0 "+$ $\lambda$; hence $l=\lambda$.

Case II: $\mathbf{v} \neq 0$. In this case, we prove that $\mathbf{v}$ belongs to the critical cone $C_{\bar{u}}$, and then with Corollary 5.7 we deduce again that $l>0$. First, we pass to the limit in $j^{\prime}\left(\overline{\mathbf{u}} ; \mathbf{v}_{\sigma}\right)$. To this end we follow (5.7). The weak convergence $\mathbf{v}_{\sigma} \rightarrow \mathbf{v}$ in $L^{2}\left(0, T ; \mathbf{L}^{2}(\Omega)\right)$ implies for $1 \leq j \leq 3$ that

$$
\lim _{|\sigma| \rightarrow 0} \int_{\Omega_{T, j}^{+}} v_{j, \sigma} d x d t=\int_{\Omega_{T, j}^{+}} v_{j} d x d t \text { and } \lim _{|\sigma| \rightarrow 0} \int_{\Omega_{T, j}^{-}} v_{j, \sigma} d x d t=\int_{\Omega_{T, j}^{-}} v_{j} d x d t
$$

Copyright (c) by SIAM. Unauthorized reproduction of this article is prohibited. 
On the other hand, using (5.19), (5.23), (5.2), and the equalities

$$
\operatorname{sign}\left(v_{j, \sigma}(t, x)\right)=\operatorname{sign}\left(\bar{u}_{j, \sigma}(t, x)\right)=\operatorname{sign}\left(\bar{\zeta}_{j, \sigma}(t, x)\right) \text { for }(t, x) \in \Omega_{T, j}^{0},
$$

we obtain

$$
\begin{aligned}
& \int_{\Omega_{T, j}^{0}}\left|v_{j}\right| d x d t \leq \liminf _{|\sigma| \rightarrow 0} \int_{\Omega_{T, j}^{0}}\left|v_{j, \sigma}\right| d x d t \leq \limsup _{|\sigma| \rightarrow 0} \int_{\Omega_{T, j}^{0}}\left|v_{j, \sigma}\right| d x d t \\
& =\limsup _{|\sigma| \rightarrow 0} \int_{\Omega_{T, j}^{0}} \bar{\zeta}_{j, \sigma} v_{j, \sigma} d x d t=\int_{\Omega_{T, j}^{0}} \bar{\zeta}_{j} v_{j} d x d t \leq \int_{\Omega_{T, j}^{0}}\left|v_{j}\right| d x d t,
\end{aligned}
$$

which implies

$$
\lim _{|\sigma| \rightarrow 0} \int_{\Omega_{T, j}^{0}}\left|v_{j, \sigma}\right| d x d t=\int_{\Omega_{T, j}^{0}}\left|v_{j}\right| d x d t=\int_{\Omega_{T, j}^{0}} \bar{\zeta}_{j} v_{j} d x d t .
$$

All together, these prove that

$$
\lim _{|\sigma| \rightarrow 0} j^{\prime}\left(\overline{\mathbf{u}} ; \mathbf{v}_{\sigma}\right)=j^{\prime}(\overline{\mathbf{u}} ; \mathbf{v})=\int_{\Omega_{T}} \boldsymbol{\zeta} \mathbf{v} d x d t .
$$

Since $\mathbf{v}$ satisfies (5.10) because every $\mathbf{v}_{\sigma}$ does, the above identity and (5.11) imply

$$
J^{\prime}(\overline{\mathbf{u}}) \mathbf{v}+\kappa j^{\prime}(\overline{\mathbf{u}} ; \mathbf{v})=\int_{\Omega_{T}}(\bar{\varphi}+\lambda \overline{\mathbf{u}}+\kappa \overline{\boldsymbol{\zeta}}) \mathbf{v} d x d t \geq 0 .
$$

To conclude that $\mathbf{v} \in C_{\overline{\mathbf{u}}}$, we prove the opposite inequality. Let us take $\mathbf{u}_{\sigma}$ as above. Then from (5.18), we obtain

$$
\int_{0}^{T} \int_{\Omega_{h}}\left(\overline{\boldsymbol{\varphi}}_{\sigma}+\lambda \overline{\mathbf{u}}_{\sigma}+\kappa \overline{\boldsymbol{\zeta}}_{\sigma}\right)\left(\mathbf{u}_{\sigma}-\overline{\mathbf{u}}_{\sigma}\right) d x d t \geq 0
$$

From here we get

$$
\int_{0}^{T} \int_{\Omega_{h}}\left(\overline{\boldsymbol{\varphi}}_{\sigma}+\lambda \overline{\mathbf{u}}_{\sigma}+\kappa \overline{\boldsymbol{\zeta}}_{\sigma}\right)\left(\overline{\mathbf{u}}-\overline{\mathbf{u}}_{\sigma}\right) d x d t+\int_{0}^{T} \int_{\Omega_{h}}\left(\overline{\boldsymbol{\varphi}}_{\sigma}+\lambda \overline{\mathbf{u}}_{\sigma}+\kappa \overline{\boldsymbol{\zeta}}_{\sigma}\right)\left(\mathbf{u}_{\sigma}-\overline{\mathbf{u}}\right) d x d t \geq 0
$$

Dividing by $\left\|\overline{\mathbf{u}}_{\sigma}-\overline{\mathbf{u}}\right\|_{L^{2}\left(0, T ; \mathbf{L}^{2}(\Omega)\right)}$, we infer

$$
\begin{gathered}
-\int_{0}^{T} \int_{\Omega_{h}}\left(\overline{\boldsymbol{\varphi}}_{\sigma}+\lambda \overline{\mathbf{u}}_{\sigma}+\kappa \overline{\boldsymbol{\zeta}}_{\sigma}\right) \mathbf{v}_{\sigma} d x d t \\
+\int_{0}^{T} \int_{\Omega_{h}}\left(\overline{\boldsymbol{\varphi}}_{\sigma}+\lambda \overline{\mathbf{u}}_{\sigma}+\kappa \overline{\boldsymbol{\zeta}}_{\sigma}\right) \frac{\mathbf{u}_{\sigma}-\overline{\mathbf{u}}}{\left\|\overline{\mathbf{u}}_{\sigma}-\overline{\mathbf{u}}\right\|_{L^{2}\left(0, T ; \mathbf{L}^{2}(\Omega)\right)}} d x d t \geq 0 .
\end{gathered}
$$

Now, using (5.25) and the estimate

$$
\left\|\mathbf{u}_{\sigma}-\overline{\mathbf{u}}\right\|_{L^{2}\left(0, T ; \mathbf{L}^{2}(\Omega)\right)} \leq C h
$$

(see [5, Lem. 4.17]), as well as (5.23), we deduce

$$
\begin{gathered}
\int_{0}^{T} \int_{\Omega_{h}}(\overline{\boldsymbol{\varphi}}+\lambda \overline{\mathbf{u}}+\kappa \overline{\boldsymbol{\zeta}}) \mathbf{v} d x d t=\lim _{|\sigma| \rightarrow 0} \int_{0}^{T} \int_{\Omega_{h}}\left(\overline{\boldsymbol{\varphi}}_{\sigma}+\lambda \overline{\mathbf{u}}_{\sigma}+\kappa \overline{\boldsymbol{\zeta}}_{\sigma}\right) \mathbf{v}_{\sigma} d x d t \\
\leq \lim _{|\sigma| \rightarrow 0}\left\|\overline{\boldsymbol{\varphi}}_{\sigma}+\lambda \overline{\mathbf{u}}_{\sigma}+\kappa \overline{\boldsymbol{\zeta}}_{\sigma}\right\|_{L^{2}\left(0, T ; \mathbf{L}^{2}\left(\Omega_{h}\right)\right)} \frac{\left\|\mathbf{u}_{\sigma}-\overline{\mathbf{u}}\right\|_{L^{2}\left(0, T ; \mathbf{L}^{2}(\Omega)\right)}}{\left\|\overline{\mathbf{u}}_{\sigma}-\overline{\mathbf{u}}\right\|_{L^{2}\left(0, T ; \mathbf{L}^{2}(\Omega)\right)}} \\
\leq C \lim _{|\sigma| \rightarrow 0} \frac{h}{\left\|\overline{\mathbf{u}}_{\sigma}-\overline{\mathbf{u}}\right\|_{L^{2}\left(0, T ; \mathbf{L}^{2}(\Omega)\right)}}=0 .
\end{gathered}
$$

Copyright $@$ by SIAM. Unauthorized reproduction of this article is prohibited. 
This completes the proof of $\mathbf{v} \in C_{\overline{\mathbf{u}}}$. Then Corollary 5.7 and the fact that $\mathbf{v} \neq 0$ lead to

$$
J^{\prime \prime}(\overline{\mathbf{u}}) \mathbf{v}^{2} \geq \delta\|\mathbf{v}\|_{L^{2}\left(0, T ; \mathbf{L}^{2}(\Omega)\right)}^{2}=\delta_{1}>0 .
$$

Therefore, with (5.33) we get

$$
\delta_{1} \leq \liminf _{|\sigma| \rightarrow 0} J^{\prime \prime}(\overline{\mathbf{u}}) \mathbf{v}_{\sigma}^{2} \leq \lim _{|\sigma| \rightarrow 0} J^{\prime \prime}\left(\mathbf{u}_{\theta}\right) \mathbf{v}_{\sigma}^{2}+\lim _{|\sigma| \rightarrow 0}\left[J^{\prime \prime}(\overline{\mathbf{u}})-J^{\prime \prime}\left(\mathbf{u}_{\theta}\right)\right] \mathbf{v}_{\sigma}^{2}=l .
$$

Hence, we have that $l \geq \min \left\{\lambda, \delta_{1}\right\}$. Now, from (5.33) we deduce the existence of $\sigma_{0}>0$ such that

$$
J^{\prime \prime}\left(\mathbf{u}_{\theta, \sigma}\right) \mathbf{v}_{\sigma}^{2} \geq \frac{l}{2} \quad \forall|\sigma| \leq \sigma_{0}
$$

or, equivalently,

$$
J^{\prime \prime}\left(\mathbf{u}_{\theta, \sigma}\right)\left(\overline{\mathbf{u}}_{\sigma}-\overline{\mathbf{u}}\right)^{2} \geq \frac{l}{2}\left\|\overline{\mathbf{u}}_{\sigma}-\overline{\mathbf{u}}\right\|_{L^{2}\left(0, T ; \mathbf{L}^{2}(\Omega)\right)}^{2} \forall|\sigma| \leq \sigma_{0} .
$$

Thus, (5.32) holds with $\delta_{0}=\min \left\{\lambda, \delta_{1}\right\} / 2$.

6. Concluding remarks. In this paper, we have considered an alternative formulation of the classical tracking control problem for 3D flows, which ensures that the optimal states are strong solutions of the Navier-Stokes system. As a consequence, we have been able to carry out a complete theoretical and numerical analysis of the optimal control problem. In particular, error estimates for the numerical discretization of the same order of the $2 \mathrm{D}$ case have been obtained. We emphasize that our analysis is applicable without assuming any smallness assumption on the data of our problem, and it can be also used to deal with sparse control problems.

The classical formulation of the control problem uses the $L^{2}$ norm of $\mathbf{y}-\mathbf{y}_{d}$. It is easy to prove the existence of at least one solution $(\overline{\mathbf{u}}, \overline{\mathbf{y}})$ for this formulation. If we make the assumption that $\bar{y}$ is a strong solution of the Navier-Stokes system, then we can follow the approach described in this paper to obtain the same results. Hence, our formulation can be regarded as a way to guarantee that $\overline{\mathbf{y}}$ is indeed a strong solution.

\section{REFERENCES}

[1] F. Abergel and R. Temam, On some control problems in fluid mechanics, Theoret. Comput. Fluid Dynamics, 1 (1990), pp. 303-325.

[2] F. Bonnans and A. Shapiro, Perturbation Analysis of Optimization Problems, SpringerVerlag, Berlin, 2000.

[3] J. Borwein and J. Vanderwerff, Convex Functions. Constructions, Characterizations and Counterexamples, Cambridge University Press, Cambridge, UK, 2010.

[4] E. CASAS, An optimal control problem governed by the evolution Navier-Stokes equations, in Optimal Control of Viscous Flow, S. S. Sritharan, ed., SIAM, Philadelphia, 1998, pp. 79-95.

[5] E. Casas And K. Chrysafinos, A discontinuous Galerkin time-stepping scheme for the velocity tracking problem, SIAM J. Numer. Anal., 50 (2012), pp. 2281-2306.

[6] E. Casas, R. Herzog, and G. Wachsmuth, Optimality conditions and error analysis of semilinear elliptic control problems with $L^{1}$ cost functional, SIAM J. Optim., 22 (2012), pp. 795820.

[7] E. Casas and F. Tröltzsch, Second-order and stability analysis for state-constrained elliptic optimal control problems with sparse controls, SIAM J. Control Optim., 52 (2014), pp. 1010-1033.

[8] F. Clarke, Optimization and Nonsmooth Analysis, John Wiley \& Sons, Toronto, 1983. 
[9] K. Deckelnick And M. Hinze, Semidiscretization and error estimates for distributed control of the instationary Navier-Stokes equations, Numer. Math., 97 (2004), pp. 297-320.

[10] P. Girault and P. Raviart, Finite Element Methods for Navier-Stokes Equations. Theory and Algorithms, Springer-Verlag, Berlin, 1986.

[11] M. D. Gunzburger And S. Manservisi, The velocity tracking problem for Navier-Stokes flows with bounded distributed control, SIAM J. Control Optim., 37 (1999), pp. 1913-1945.

[12] M. D. Gunzburger and S. Manservisi, Analysis and approximation of the velocity tracking problem for Navier-Stokes flows with distributed control, SIAM J. Numer. Anal., 37 (2000), pp. 1481-1512.

[13] R. Herzog, G. Stadler, and G. Wachsmuth, Directional sparsity in optimal control of partial differential equations, SIAM J. Control Optim., 50 (2012), pp. 943-963.

[14] M. Hinze And K. Kunisch, Second order methods for optimal control of time-dependent fluid flow, SIAM J. Control Optim., 40 (2001), pp. 925-946.

[15] O. A. Ladyzhenskaya, The Mathematical Theory of Viscous Incompressible Flow, Second English edition, revised and enlarged. Translated from the Russian by R. A. Silverman and J. Chu, Math. Appl. 2, Gordon and Breach Science Publishers, New York, 1969.

[16] J. Lions, Quelques Méthodes de Résolution des Problèmes aux Limites non Linéaires, Dunod, Paris, 1969

[17] J. Lions and E. Magenes, Problèmes aux Limites non Homogènes, Vols. 1 \& 2, Dunod, Paris, 1968.

[18] H. Sohr And W. Wahl, Generic solvability of the equations of Navier-Stokes, Hiroshima Math. J., 17 (1987), pp. 613-625.

[19] V. Solonnikov, Estimates for solutions of nonstationary Navier-Stokes equations, J. Soviet Math., 8 (1977), pp. 213-317.

[20] G. Stadler, Elliptic optimal control problems with $L^{1}$-control cost and applications for the placement of control devices, Comput. Optim. Appl., 44 (2009), pp. 159-181.

[21] R. Temam, Navier-Stokes Equations, North-Holland, Amsterdam, 1979.

[22] F. Tröltzsch and D. WachSmuth, Second-order sufficient optimality conditions for the optimal control of Navier-Stokes equations, ESAIM Control Optim. Calc. Var., 12 (2006), pp. 93-119.

[23] G. Wachsmuth and D. Wachsmuth, Convergence and regularisation results for optimal control problems with sparsity functional, ESAIM Control Optim. Calc. Var., 17 (2011), pp. $858-886$.

[24] N. J. Walkington, Compactness properties of the DG and $C G$ time stepping schemes for parabolic equations, SIAM J. Numer. Anal., 47 (2010), pp. 4680-4710.

Copyright (c) by SIAM. Unauthorized reproduction of this article is prohibited. 